\title{
Boundary terms and three-point functions: an AdS/CFT puzzle resolved
}

\author{
Daniel Z. Freedman, ${ }^{a, b}$ Krzysztof Pilch, ${ }^{c}$ Silviu S. Pufu ${ }^{e}$ and Nicholas P. Warner ${ }^{c, d}$ \\ ${ }^{a}$ SITP and Department of Physics, Stanford University, \\ Stanford, CA 94305, U.S.A. \\ ${ }^{b}$ Center for Theoretical Physics and Department of Mathematics, \\ Massachusetts Institute of Technology, \\ Cambridge, MA 02139, U.S.A. \\ ${ }^{c}$ Department of Physics and Astronomy, University of Southern California. \\ Los Angeles, CA 90089, U.S.A. \\ ${ }^{d}$ Department of Mathematics, University of Southern California, \\ Los Angeles, CA 90089, U.S.A. \\ e Joseph Henry Laboratories, Princeton University, \\ Princeton, NJ 08544, U.S.A. \\ E-mail: dzfmit@gmail.com, pilch@usc.edu, spufu@princeton.edu, \\ warner@usc . edu
}

ABStRACT: $\mathcal{N}=8$ superconformal field theories, such as the ABJM theory at ChernSimons level $k=1$ or 2 , contain 35 scalar operators $\mathcal{O}_{I J}$ with $\Delta=1$ in the $\mathbf{3 5}_{v}$ representation of $\mathrm{SO}(8)$. The 3-point correlation function of these operators is non-vanishing, and indeed can be calculated non-perturbatively in the field theory. But its $\mathrm{AdS}_{4}$ gravity dual, obtained from gauged $\mathcal{N}=8$ supergravity, has no cubic $A^{3}$ couplings in its Lagrangian, where $A^{I J}$ is the bulk dual of $\mathcal{O}_{I J}$. So conventional Witten diagrams cannot furnish the field theory result. We show that the extension of bulk supersymmetry to the $\mathrm{AdS}_{4}$ boundary requires the introduction of a finite $A^{3}$ counterterm that does provide a perfect match to the 3-point correlator. Boundary supersymmetry also requires infinite counterterms which agree with the method of holographic renormalization. The generating functional of correlation functions of the $\Delta=1$ operators is the Legendre transform of the on-shell action, and the supersymmetry properties of this functional play a significant role in our treatment.

KEYwords: AdS-CFT Correspondence, Extended Supersymmetry, Supergravity Models

ARXIV EPRINT: 1611.01888 


\section{Contents}

1 Introduction 1

2 Field theory computations $\quad 4$

2.1 Dimension-1 scalar operators in 3d SCFTs 4

2.2 Application to $\mathcal{N}=8$ SCFTs $\quad 6$

2.2.1 Summary 9

$\begin{array}{lll}2.2 .2 & \text { An example } & 10\end{array}$

3 Boundary terms in $\mathcal{N}=1$ truncations $\quad 10$

3.1 Review of the Bogomolny argument in [14] 10

$\begin{array}{lll}3.2 & \text { Boundary terms required by supersymmetry } & 11\end{array}$

3.3 The global limit of $\mathcal{N}=1, \mathrm{AdS}_{4}$ supergravity 12

$\begin{array}{lll}3.4 & \text { Further conventions and asymptotic behavior } & 15\end{array}$

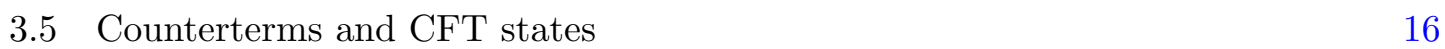

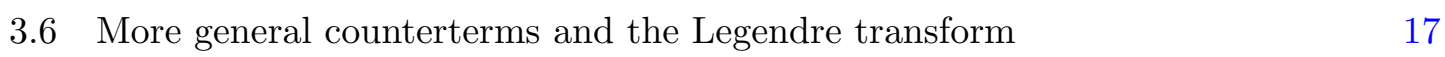

3.7 Cancellation of the supersymmetry variation of the on-shell action 20

3.8 The $A B^{2}$ boundary term: a minor puzzle resolved 21

3.9 Boundary SUSY for $\mathcal{N}=1$ truncations of supergravity. 23

42 - and 3-point correlators from $\mathcal{N}=1$ supergravity $\quad 23$

4.12 - and 3-point correlators 23

4.2 On the nonlinear boundary condition for $A(r, \vec{x}) \quad 25$

5 The $\mathcal{N}=8$ supergravity $\quad 26$

6 Bogomolny argument in $\mathcal{N}=8$ supergravity 29

$\begin{array}{ll}6.1 \text { Motivation } & 29\end{array}$

6.2 The BPS equations in the "kink Ansatz" 29

$\begin{array}{lll}6.3 & \text { Completing the square } & 30\end{array}$

6.4 Collecting the boundary terms 32

6.5 Asymptotics and counterterms 33

7 Boundary sources and $\mathcal{N}=8$ supersymmetry $\quad 34$

7.1 Preliminaries 34

7.2 The boundary variation from the bulk $\mathcal{N}=8$ action 37

$\begin{array}{lll}7.3 & \text { Adding counterterms } & 38\end{array}$

8 2- and 3-point correlators from $\mathcal{N}=8$ supergravity $\quad 40$

8.1 The counterterms in the $\mathrm{SL}(8, \mathbb{R})$ basis $\quad 40$

8.2 The correlators of the operators $\mathcal{O}_{I J}(\vec{x}) \quad 41$

$\begin{array}{lll}9 & \text { Conclusions } & 42\end{array}$ 
A An alternative computation of 3-point functions of dimension-1 operators

B Some details of the derivation of (3.20) 46

$\begin{array}{ll}\text { C Truncating the } \mathcal{N}=8 \text { theory } & 47\end{array}$

$\begin{array}{lll}\text { C.1 Truncations and flows } & 47\end{array}$

$\begin{array}{ll}\text { C.2 Calculation of the counterterms } & 48\end{array}$

D Some identities for $\mathrm{SO}(8)$ (anti-)self-dual tensors 49

E The $\mathrm{U}(1)^{3}$-invariant truncation in [14] 50

\section{Introduction}

The anti-de Sitter/conformal field theory (AdS/CFT) duality has passed many tests. When precise comparisons of gravity and field theory results can be made, the results generally agree. This paper focuses on an aspect of the $\mathrm{AdS}_{4} / \mathrm{CFT}_{3}$ duality in which there is an apparent acute conflict between the two sides of the duality, but we find that the conflict is resolved through oft-neglected boundary terms.

The conflict involves the holographic computation of the 3-point function of dimension1 operators of the $\mathrm{CFT}_{3}$. For concreteness, let us describe it in the case of the maximally supersymmetric $(\mathcal{N}=8) 3$ d superconformal field theories (SCFTs) whose holographic description includes four-dimensional, $\mathcal{N}=8$ gauged supergravity [1]. The representation theory of the $\mathcal{N}=8$ superconformal algebra shows that any $3 \mathrm{~d}$, local $\mathcal{N}=8$ SCFT must contain scalar operators $\mathcal{O}_{I J}, 1 \leq I, J \leq 8$ transforming in the traceless symmetric tensor description of the $\mathbf{3 5}_{v}$ representation of the $\mathrm{SO}(8)$ global R-symmetry group with scale dimension $\Delta=1$. These scalars are present in any local $\mathcal{N}=8$ SCFT because they belong to the same superconformal multiplet as the stress tensor. As we will explain, superconformal Ward identities imply that the 3-point correlation function $\left\langle\mathcal{O}_{I J}\left(\vec{x}_{1}\right) \mathcal{O}_{J K}\left(\vec{x}_{2}\right) \mathcal{O}_{K I}\left(\vec{x}_{3}\right)\right\rangle$ for given $I, J$ and $K$ (no sum) must be non-vanishing and related to the 2-point function of the canonically normalized stress tensor. This 2-point function can be calculated exactly using supersymmetric localization [2] whenever an explicit Lagrangian description is available. The AdS/CFT correspondence requires the 3-point functions $\left\langle\mathcal{O}_{I J}\left(\vec{x}_{1}\right) \mathcal{O}_{J K}\left(\vec{x}_{2}\right) \mathcal{O}_{K I}\left(\vec{x}_{3}\right)\right\rangle$ be matched by a calculation in the gravity bulk, where 3-point functions are usually calculated by evaluating a Witten diagram containing a cubic vertex from the bulk Lagrangian. The problem is that the Lagrangian of $\mathcal{N}=8$ gauged supergravity in four dimensions does not contain any cubic scalar couplings! Thus another way to obtain $\left\langle\mathcal{O}_{I J}\left(\vec{x}_{1}\right) \mathcal{O}_{J K}\left(\vec{x}_{2}\right) \mathcal{O}_{K I}\left(\vec{x}_{3}\right)\right\rangle$ must be found.

Note that the foregoing description of the conflict does not rely on a specific field theory realization of the $\mathcal{N}=8$ SCFT dual to four-dimensional, $\mathcal{N}=8$ gauged supergravity. In fact, the four-dimensional, $\mathcal{N}=8$ gauged supergravity theory does not correspond to a 
unique $\mathcal{N}=8 \mathrm{SCFT}$; it corresponds instead to a universal sector describing the stress tensor multiplet of all known $\mathcal{N}=8$ SCFTs with holographic duals. These are the large $N$ limits of three distinct families: $\mathrm{U}(N)_{1} \times \mathrm{U}(N)_{-1}$ ABJM theory [3], $\mathrm{U}(N)_{2} \times \mathrm{U}(N)_{-2}$ ABJM theory, and $\mathrm{U}(N)_{2} \times \mathrm{U}(N+1)_{-2}$ ABJ theory [4]. (See also [5-8] for earlier work that was generalized in $[3,4].)^{1}$ These $\mathcal{N}=8$ SCFTs are believed to be, respectively, the infrared limits of maximally supersymmetric 3d Yang-Mills theory with gauge group $\mathrm{U}(N), \mathrm{O}(2 N)$, and $\mathrm{O}(2 N+1)$. At large $N$ they have a dual description in terms of elevendimensional supergravity, of which four-dimensional $\mathcal{N}=8$ gauged supergravity of [1] is a consistent truncation.

An important clue to the resolution of the conflict appears in [14], where an $\mathcal{N}=2$ truncation of the $\mathcal{N}=8$ supergravity theory was studied. ${ }^{2}$ The truncation contains 3 complex scalars $z^{\alpha}=A^{\alpha}+i B^{\alpha}, \alpha=1,2,3$. The goal of [14] was to match the field theory calculation of the $S^{3}$ free energy of an $\mathcal{N}=2$-preserving mass deformation of the ABJM theory obtained in [16] by the method of supersymmetric localization developed in [17-19] (for recent reviews, see [20, 21]). Obtaining the match is not straightforward. First, the bulk scalars $A^{\alpha}$ dual to the three $\Delta=1$ field theory operators $\mathcal{O}_{\alpha}$ in the truncation ${ }^{3}$ must be quantized by alternate quantization [22]. Second, the infinite counterterms obtained from the method of holographic renormalization must be supplemented by a finite counterterm. Both alternate quantization and the finite counterterm [23, 24] are required by the supersymmetry of the Legendre transformed on-shell action which is the generator of correlation functions in the boundary field theory [25]. We focus on the counterterm obtained in [14] by a Bogomolny factorization argument for the action of planar domain walls [26]. The counterterm turns out to be proportional to $\int d^{3} x \sqrt{-h} A^{1} A^{2} A^{3}$ with a determined coefficient. ${ }^{4}$ It turns out that this boundary term and its extension to the full $\mathcal{N}=8$ theory are exactly what we need to compute $\left\langle\mathcal{O}_{1}\left(\vec{x}_{1}\right) \mathcal{O}_{2}\left(\vec{x}_{2}\right) \mathcal{O}_{3}\left(\vec{x}_{3}\right)\right\rangle$ and $\left\langle\mathcal{O}_{I J}\left(\vec{x}_{1}\right) \mathcal{O}_{J K}\left(\vec{x}_{2}\right) \mathcal{O}_{K I}\left(\vec{x}_{3}\right)\right\rangle$.

The main effort in this paper is to obtain the essential cubic counterterm ${ }^{5}$ by modifying the bulk theory so that supersymmetry extends to the boundary. The principle we employ is that the on-shell supergravity action, seen via the AdS/CFT dictionary as a functional of the sources for the field theory operators, should be supersymmetric. We analyze this first at the level of a limit of four-dimensional $\mathcal{N}=1$ supergravity in which the back-reaction of

\footnotetext{
${ }^{1}$ The $\mathrm{ABJ}(\mathrm{M})$ theory $[3,4]$ is a $\mathrm{U}(N)_{k} \times \mathrm{U}(M)_{-k}$ Chern-Simons matter theory in three dimensions that has only $\mathcal{N}=6$ manifest supersymmetry. It is the effective theory on $N$ coincident M2-branes placed at the singular point of a certain $\mathbb{C}^{4} / \mathbb{Z}_{k}$ orbifold. When $k=1$ or 2 and $M=N$ or $M=N+1$, the infrared limit is believed to have enhanced $\mathcal{N}=8$ supersymmetry [3, 9-13]. The $\mathrm{U}(N)_{1} \times \mathrm{U}(N+1)_{-1}$ theory is dual to the $\mathrm{U}(N)_{1} \times \mathrm{U}(N)_{-1}$ one, so there are only three distinct families of $\mathcal{N}=8$ SCFTs of this type. A fourth family of $\mathcal{N}=8$ SCFTs is given by the $\mathrm{SU}(2)_{k} \times \mathrm{SU}(2)_{-k}$ BLG theories [5-8] but do not have classical supergravity duals. The BLG theories have manifest $\mathcal{N}=8$ supersymmetry.

${ }^{2}$ To our knowledge, this truncation was first given in [15].

${ }^{3}$ The 3 operators $\mathcal{O}_{\alpha}$ constitute the subset of the $35 \mathcal{O}_{I J}$ that is part of the truncated theory. This subset is defined in the next section.

${ }^{4}$ The induced metric at the boundary is $h_{a b}$.

${ }^{5}$ It is well known that a boundary term quadratic in fermion fields must be added to the bulk action in order to obtain the 2-point correlator of fermion operators in the boundary theory, [27-30]. Also, a cubic boundary counterterm plays a role in the holographic story of extremal correlation functions in $\mathcal{N}=4 \mathrm{SYM}$ theory [31]. See, also [32-34].
} 
matter fields on the $\mathrm{AdS}_{4}$ background is consistently suppressed and the resulting theory, similar to that of [36], enjoys global AdS supersymmetry. We also discuss the changes needed to extend the treatment to $\mathcal{N}=1$ supergravity. Then we move on to $\mathcal{N}=8$ and show how the cubic counterterm emerges from an extended Bogomolny argument and finally how it is generated in the full $\mathcal{N}=8$ gauged supergravity. In both the $\mathcal{N}=1$ and $\mathcal{N}=8$ analyses, the alternate quantization of [25], implemented through a Legendre transform of the on-shell action, plays an important role. It is worth noting that for $\mathcal{N}=1$ global supersymmetric theories with boundaries, the boundary terms we find here (and their derivation) are in some ways very similar to those encountered in lower dimensions [37-39].

We should emphasize that the framework developed here goes beyond the immediate application to the correspondence between the $\mathcal{N}=8$ gauged supergravity and its maximally supersymmetric 3d SCFT dual. Indeed, any holographic computation of a 3-point correlator of dimension-1 operators in a $3 \mathrm{~d}$ CFT with a gravity dual must be similar to the present study in that the bulk cubic vertex must vanish ${ }^{6}$ and the answer comes from a (super)gravity boundary term. ${ }^{7}$ Our claim is that in a four-dimensional $\mathcal{N} \geq 1$ supergravity theory this boundary term can be determined from the requirement that the theory is supersymmetric, including boundary terms.

It is worth contrasting the situation here to that of four-dimensional $\mathcal{N}=4$ supersymmetric Yang-Mills theory, where the 3-point correlator of the chiral primary operator $\mathcal{O}_{\Delta=2}$ in the same multiplet as the stress tensor is protected $[43,44]$. This means that it is independent of the gauge coupling constant, and so it can be computed at weak coupling by performing Wick contractions. This is not true for the scalars $\mathcal{O}_{I J}$ of $\mathcal{N}=8$ SCFTs, where there are strong coupling effects. It is worth displaying the result for the supergravity limits of the 2- and 3-point function of the operators $\mathcal{O}_{\alpha}$ in the truncation of [14]:

$$
\begin{aligned}
\left\langle\mathcal{O}_{\alpha}\left(\vec{x}_{1}\right) \mathcal{O}_{\beta}\left(\vec{x}_{2}\right)\right\rangle & =\frac{L^{2}}{2 \pi^{3} G_{4}} \frac{\delta_{\alpha \beta}}{\left|\vec{x}_{12}\right|^{2}}=\frac{\sqrt{2} N^{3 / 2} k^{1 / 2}}{3 \pi^{3}} \frac{\delta_{\alpha \beta}}{\left|\vec{x}_{12}\right|^{2}}, \\
\left\langle\mathcal{O}_{1}\left(\vec{x}_{1}\right) \mathcal{O}_{2}\left(\vec{x}_{2}\right) \mathcal{O}_{3}\left(\vec{x}_{3}\right)\right\rangle & =\frac{L^{2}}{4 \pi^{4} G_{4}} \frac{1}{\left|\vec{x}_{12}\right|\left|\vec{x}_{23}\right|\left|\vec{x}_{31}\right|}=\frac{\sqrt{2} N^{3 / 2} k^{1 / 2}}{6 \pi^{4}} \frac{1}{\left|\vec{x}_{12}\right|\left|\vec{x}_{23}\right|\left|\vec{x}_{31}\right|} .
\end{aligned}
$$

In these expressions, $L$ is the radius of the dual $\mathrm{AdS}_{4}$ solution, $G_{4}$ is the effective fourdimensional Newton constant, $\vec{x}_{i j} \equiv \vec{x}_{i}-\vec{x}_{j}, N$ was defined above, and $k=1$ or 2 is the Chern-Simons level of the ABJ(M) theory. We will first explain how to derive (1.1) in the $\mathcal{N}=8$ ABJM theory based on previous results that use supersymmetric localization

\footnotetext{
${ }^{6}$ Suppose that the on-shell action did contain an $A^{3}$ or $A \partial_{\mu} A \partial^{\mu} A$ vertex. It is curious to note that the results for the Witten diagrams given in [40] are both infinite when $d=3$, and $\Delta_{1}=\Delta_{2}=\Delta_{3}=1$.

${ }^{7} \mathrm{An}$ interesting example of dimension-1 operators in a non-supersymmetric instance of $\mathrm{AdS}_{4} / \mathrm{CFT}_{3}$ is present in the higher spin $/ O(N)$ vector model duality conjectured in [41]. For this model, the dimension-1 scalar operators have $s=0$ for the higher spin currents of spin $s$. The match of 3-point functions of higherspin currents between field theory and holography was performed in [42] for all $s$. For $s=0$, the authors of [42] argued for a match of the 3-point function of dimension-1 scalar operators somewhat indirectly by considering the analytic continuation of the result for arbitrary $s$, and not by explicitly computing a boundary term as we do here. Perhaps one can provide a more direct argument by explicitly computing the required boundary term by imposing the condition that the higher spin symmetry should extend to the boundary.
} 
and then derive it from $\mathcal{N}=8$ supergravity. Equality of the coefficients follows from the AdS/CFT dictionary.

Therefore, in addition to uncovering the essential role of supergravity boundary terms in the computation of CFT three-point functions, the results presented in this paper also provide another precision test of holography: the equality in (1.1).

The rest of this paper is organized as follows. In section 2 we review the field theory computations of correlation functions of dimension- 1 operators. In section 3 we start with a toy example in $\mathcal{N}=1$ supergravity in 4 dimensions, in which we derive the boundary terms needed to ensure supersymmetry. In section 4 we use these boundary terms to calculate holographically the 3-point function of dimension-1 operators, thus resolving the puzzle mentioned above in an $\mathcal{N}=1$ toy example. In sections $5-8$ we generalize this computation to $\mathcal{N}=8$ gauged supergravity: we start with a brief review in section 5 , we develop a Bogomolny argument that motivates the presence of a boundary term in section 6 , we use this boundary term to verify supersymmetry in section 7 , and we perform the holographic computation of the 3-point functions of dimension-1 scalar operators in section 8 . We end with concluding remarks in section 9 .

\section{Field theory computations}

In this section we discuss 3-point functions of dimension-1 scalar operators from a field theory perspective. We start in section 2.1 with a general discussion of dimension-1 scalar operators in $3 \mathrm{~d}$ SCFTs. In section 2.2 we then specialize to $\mathcal{N}=8$ SCFTs, which are the main focus of this paper.

\subsection{Dimension-1 scalar operators in 3d SCFTs}

In 3d SCFTs with at least $\mathcal{N}=2$ supersymmetry, scalar operators of dimension 1 are very common. Indeed, these operators appear in one of two ways: either as part of a chiral or anti-chiral multiplet, where they carry R-charge 1 or -1 , respectively, or as part of the same multiplet as a conserved flavor symmetry current, where they have vanishing R-charge. There are no other multiplets of the $\mathcal{N}=2$ superconformal algebra that contain dimension-1 scalar operators. Of course, not every $\mathcal{N}=2$ SCFT must have a chiral or anti-chiral operator of dimension 1 , but if there are any flavor symmetries present, then dimension-1 operators must be present as part of the conserved flavor current multiplets.

When we consider extended supersymmetry, dimension-1 scalar operators can, of course, only arise in multiplets that upon reduction to $\mathcal{N}=2$ contain either a flavor current multiplet, a chiral multiplet of R-charge 1, or an anti-chiral multiplet of R-charge -1 . This always happens, for instance, in SCFTs with $\mathcal{N} \geq 4$ supersymmetry. Indeed, in such SCFTs some of the R-symmetry currents (which are in the same $\mathcal{N} \geq 4$ supermultiplet as the stress tensor) can be interpreted as flavor currents upon reduction to $\mathcal{N}=2$, and these flavor currents belong to $\mathcal{N}=2$ supermultiplets also containing dimension- 1 scalar operators. Therefore, local $\mathcal{N} \geq 4$ SCFTs must always contain scalar operators of dimension 1 that belong to the same $\mathcal{N} \geq 4$ supermultiplet as the stress energy tensor. 
In $\mathcal{N}=2$ SCFTs, supersymmetry techniques allow for the computation of certain 3 -point functions of dimension-1 scalar operators exactly. Without extended supersymmetry, the 3-point functions that are calculable with existing supersymmetric localization techniques are those of precisely one chiral operator, one anti-chiral operator, and one operator in a conserved flavor current multiplet. Such a 3-point function is non-vanishing only if the chiral and anti-chiral operators carry non-vanishing charges under the flavor symmetry corresponding to the third operator, and in this discussion we will assume this. The other type of non-zero three point function, namely between three operators in conserved current multiplets, does not seem to be accessible through supersymmetric localization in theories with just $\mathcal{N}=2$ supersymmetry, but it can of course also be computed in theories with extended supersymmetry in which supersymmetry relates it to a chiral-anti-chiral-conserved current 3-point function.

To be precise, consider a dimension- 1 chiral operator $\mathcal{O}$, an anti-chiral operator $\overline{\mathcal{O}}$, and a dimension-1 real operator $J$ in the same multiplet as a conserved flavor current $j^{\mu}$. Let the operators $\mathcal{O}$ and $\overline{\mathcal{O}}$ have charges $q$ and $-q$, respectively, under the symmetry generated by $j^{\mu}$. It is important to be precise about the normalization of these operators. For the chiral and anti-chiral operators, let us normalize them such that

$$
\langle\mathcal{O}(\vec{x}) \overline{\mathcal{O}}(0)\rangle=\frac{1}{8 \pi^{2}|\vec{x}|^{2}} .
$$

It is convenient to normalize $J$ such that it is related to the canonically normalized $j^{\mu}$ in a canonical way. Canonical normalization of $j^{\mu}$ means that the following OPE holds

$$
j^{\mu}(\vec{x}) \mathcal{O}(0)=q \frac{x^{\mu}}{4 \pi|\vec{x}|^{3}} \mathcal{O}(0)+\ldots
$$

We take the canonical normalization of $J$ to mean that if the conserved current $j^{\mu}$ is normalized as in (2.2), then $J$ should be normalized such that it gives the OPE

$$
J(\vec{x}) \mathcal{O}(0)=q \frac{1}{4 \pi|\vec{x}|} \mathcal{O}(0)+\ldots
$$

With this normalization, we have the following 2-point functions at separated points

$$
\langle J(\vec{x}) J(0)\rangle=\frac{\tau}{16 \pi^{2}|\vec{x}|^{2}}, \quad\left\langle j^{\mu}(\vec{x}) j^{\nu}(0)\right\rangle=\frac{\tau}{16 \pi^{2}}\left(\partial_{\lambda} \partial^{\lambda} \eta^{\mu \nu}-\partial^{\mu} \partial^{\nu}\right) \frac{1}{|\vec{x}|^{2}} .
$$

The coefficient $\tau$ can be computed using supersymmetric localization of a certain deformation of the SCFT on $S^{3}$. The deformation can be interpreted as a modification of the supersymmetry algebra where we change the R-charges of all chiral operators by adding to them the flavor charges under $j^{\mu}$ multiplied by a parameter $t$. It is possible to compute the $S^{3}$ free energy $F(t)$ for this deformation of the theory exactly. Then one extracts [45] (for recent reviews, see $[21,46]$ )

$$
\tau=-\left.\frac{2}{\pi^{2}} \frac{d^{2} F}{d t^{2}}\right|_{t=0}
$$


The 3-point function $\left\langle\mathcal{O}\left(\vec{x}_{1}\right) \overline{\mathcal{O}}\left(\vec{x}_{2}\right) J\left(\vec{x}_{3}\right)\right\rangle$ can be computed using these results very easily. Indeed, by conformal invariance, it takes the form

$$
\left\langle\mathcal{O}\left(\vec{x}_{1}\right) \overline{\mathcal{O}}\left(\vec{x}_{2}\right) J\left(\vec{x}_{3}\right)\right\rangle=\frac{\lambda_{\mathcal{O} \overline{\mathcal{O}} J}}{\left|\vec{x}_{1}-\vec{x}_{2}\right|\left|\vec{x}_{1}-\vec{x}_{3}\right|\left|\vec{x}_{2}-\vec{x}_{3}\right|} .
$$

Using the OPE (2.2) and the 2-point function (2.1), we obtain

$$
\lambda_{\mathcal{O} \overline{\mathcal{O}} J}=\frac{q}{32 \pi^{3}}
$$

The simplicity of (2.7) is misleading, because it relies on the canonical normalization of $J$ as well as on the normalization of the chiral and anti-chiral operators in (2.1). The following ratio of three and 2-point functions is a constant that is independent of the normalization of these operators:

$$
\frac{\left\langle\mathcal{O}\left(\vec{x}_{1}\right) \overline{\mathcal{O}}\left(\vec{x}_{2}\right) J\left(\vec{x}_{3}\right)\right\rangle^{2}}{\left\langle\mathcal{O}\left(\vec{x}_{1}\right) \overline{\mathcal{O}}\left(\vec{x}_{3}\right)\right\rangle\left\langle\mathcal{O}\left(\vec{x}_{3}\right) \overline{\mathcal{O}}\left(\vec{x}_{2}\right)\right\rangle\left\langle J\left(\vec{x}_{1}\right) J\left(\vec{x}_{2}\right)\right\rangle}=\frac{q^{2}}{\tau} .
$$

It depends on both the charge $q$ as well as the coefficient $\tau$ obtained through (2.5).

\subsection{Application to $\mathcal{N}=8$ SCFTs}

This framework can be applied to the computation of the 3-point function of dimension-1 operators in maximally supersymmetric $\mathcal{N}=8 \mathrm{SCFTs}$, as we now explain. As described above, any SCFT with at least $\mathcal{N}=4$ supersymmetry must have dimension- 1 scalars in the same multiplet as the stress energy tensor. In an interacting $\mathcal{N}=8$ theory, these are the only dimension- 1 operators that can exist. They transform in a 35-dimensional representation of the $\mathrm{SO}(8) \mathrm{R}$-symmetry that, by a choice of convention, we take to be the $\mathbf{3 5}_{v}$. In addition to the stress tensor and the dimension-1 scalar operators transforming in the $\mathbf{3 5}_{v}$, the $\mathcal{N}=8$ stress tensor multiplet also contains an R-symmetry current transforming in the adjoint of $\mathrm{SO}(8)$, the supercurrent of spin $3 / 2$ transforming (by a choice of conventions) in the $\boldsymbol{8}_{s}$ of $\mathrm{SO}(8)$, dimension- 2 pseudoscalars transforming in the $\mathbf{3 5}_{c}$, as well as dimension- $3 / 2$ operators of spin $1 / 2$ transforming in the $\mathbf{5 6}_{s}$.

The 2-point function of the canonically normalized stress tensor is determined by conservation and conformal invariance to be

$$
\left\langle T_{\mu \nu}(\vec{x}) T_{\rho \sigma}(0)\right\rangle=\frac{c_{T}}{64}\left(P_{\mu \rho} P_{\nu \sigma}+P_{\nu \rho} P_{\mu \sigma}-P_{\mu \nu} P_{\rho \sigma}\right) \frac{1}{16 \pi^{2}|\vec{x}|^{2}},
$$

where $P_{\mu \nu} \equiv \eta_{\mu \nu} \partial^{\lambda} \partial_{\lambda}-\partial_{\mu} \partial_{\nu}$, and $c_{T}$ is a constant that depends on the theory. This definition means that one has $c_{T}=1$ in a non-supersymmetric theory of a free massless real scalar. A straightforward computation then shows that one has $c_{T}=1$ in a nonsupersymmetric theory of a free massless Majorana fermion. The free $\mathcal{N}=8$ theory contains 8 real scalars and 8 Majorana fermions and it thus has $c_{T}=16$.

The 2-point function of the canonically normalized $\mathrm{SO}(8)$ R-symmetry current is also determined up to an overall constant by conformal invariance and conservation. Moreover 
the superconformal algebra relates this constant to $c_{T}$, and the 2-point function takes the form

$$
\left\langle j_{I J}^{\mu}(\vec{x}) j_{K L}^{\nu}(0)\right\rangle=\frac{c_{T}}{64}\left(\delta_{I K} \delta_{J L}-\delta_{I L} \delta_{J K}\right) P^{\mu \nu} \frac{1}{16 \pi^{2}|\vec{x}|^{2}}
$$

where $j_{I J}^{\mu}$ is antisymmetric in the $I J$ indices. The constant, $c_{T}$, has been computed in many examples by considering Abelian flavor currents and using the method described around (2.5). We will provide a few explicit examples shortly.

We now focus on the dimension- 1 scalar operators in the $\mathbf{3 5}_{v}$ of $\mathrm{SO}(8)$, which we will represent by a symmetric traceless tensor $\mathcal{O}_{I J}(\vec{x})$. To simplify the following formulas, it is convenient to pass to an index free notation by contracting $\mathcal{O}_{I J}$ with a traceless symmetric matrix $M^{I J}$, thus defining

$$
\mathcal{O}(\vec{x}, M)=M^{I J} \mathcal{O}_{I J}(\vec{x})
$$

The two and 3-point functions of $\mathcal{O}(\vec{x}, M)$ are restricted by conformal and $\mathrm{SO}(8)$ invariance to take the form

$$
\begin{aligned}
\left\langle\mathcal{O}\left(\vec{x}_{1}, M_{1}\right) \mathcal{O}\left(\vec{x}_{2}, M_{2}\right)\right\rangle & =c_{2} \frac{\operatorname{tr}\left(M_{1} M_{2}\right)}{\left|\vec{x}_{1}-\vec{x}_{2}\right|^{2}}, \\
\left\langle\mathcal{O}\left(\vec{x}_{1}, M_{1}\right) \mathcal{O}\left(\vec{x}_{2}, M_{2}\right) \mathcal{O}\left(\vec{x}_{3}, M_{3}\right)\right\rangle & =c_{3} \frac{\operatorname{tr}\left(M_{1} M_{2} M_{3}+M_{1} M_{3} M_{2}\right)}{\left|\vec{x}_{1}-\vec{x}_{2}\right|\left|\vec{x}_{1}-\vec{x}_{3}\right|\left|\vec{x}_{2}-\vec{x}_{3}\right|},
\end{aligned}
$$

for some constants $c_{2}$ and $c_{3}$. Of course, $c_{2}$ can be changed by changing the normalization of the operators, so it may not be meaningful, and one might want to consider instead a combination of two and three point functions that is invariant under rescalings of the operators:

$$
\begin{array}{r}
\frac{\left\langle\mathcal{O}\left(\vec{x}_{1}, M_{1}\right) \mathcal{O}\left(\vec{x}_{2}, M_{2}\right) \mathcal{O}\left(\vec{x}_{3}, M_{3}\right)\right\rangle^{2}}{\left\langle\mathcal{O}\left(\vec{x}_{1}, M_{1}\right) \mathcal{O}\left(\vec{x}_{3}, M_{2}\right)\right\rangle\left\langle\mathcal{O}\left(\vec{x}_{2}, M_{1}\right) \mathcal{O}\left(\vec{x}_{3}, M_{2}\right)\right\rangle\left\langle\mathcal{O}\left(\vec{x}_{1}, M_{3}\right) \mathcal{O}\left(\vec{x}_{2}, M_{3}\right)\right\rangle} \\
=\frac{c_{3}^{2}}{c_{2}^{3}} \frac{\left[\operatorname{tr}\left(M_{1} M_{2} M_{3}+M_{1} M_{3} M_{2}\right)\right]^{2}}{\operatorname{tr}\left(M_{1} M_{2}\right) \operatorname{tr}\left(M_{1} M_{2}\right) \operatorname{tr}\left(M_{3} M_{3}\right)} .
\end{array}
$$

In order to connect (2.12)-(2.13) with the discussion of the previous section, which considered $\mathcal{N}=2$ SCFTs, we should understand how the $\mathbf{3 5}_{v}$ operators $\mathcal{O}_{I J}$ transform under an $\mathcal{N}=2$ superconformal subalgebra of the $\mathcal{N}=8$ algebra. One can choose an embedding of the $\mathcal{N}=2$ superconformal algebra $\mathfrak{o s p}(2 \mid 4)$ into $\mathfrak{o s p}(8 \mid 4)$ such that the $\mathcal{N}=2 \mathrm{SO}(2)_{R} \mathrm{R}$-symmetry is generated by the anti-Hermitian $8 \times 8$ matrix

$$
R=\frac{i}{2}\left(\begin{array}{cccc}
\sigma_{2} & 0 & 0 & 0 \\
0 & \sigma_{2} & 0 & 0 \\
0 & 0 & \sigma_{2} & 0 \\
0 & 0 & 0 & \sigma_{2}
\end{array}\right)
$$

acting in the $\boldsymbol{8}_{v}$ representation of $\mathrm{SO}(8)$. In other words, the $\mathcal{N}=2 \mathrm{R}$-symmetry current is $j^{\mu} \equiv R^{I J} j_{I J}^{\mu}$. It is not hard to see that the $\mathbf{3 5}_{v}$ operators have the following $\mathcal{N}=2$ R-charges: ten of them have R-charge 1 and are thus chiral operators from the $\mathcal{N}=2$ point 
of view; ten of them have R-charge -1 and are thus anti-chiral operators from the $\mathcal{N}=2$ point of view; and fifteen of them have vanishing $\mathrm{R}$-charge and therefore belong to flavor current multiplets from the $\mathcal{N}=2$ point of view. Indeed, from an $\mathcal{N}=2$ perspective, the flavor symmetry is $\mathrm{SU}(4)$, because this is the subgroup of $\mathrm{SO}(8)$ that commutes with (2.14). Since SU(4) has rank three, there are three commuting Abelian flavor currents that can be taken to correspond to the $\mathrm{SO}(8)$ generators:

$$
\begin{aligned}
F_{(1)} & =\frac{i}{2}\left(\begin{array}{cccc}
\sigma_{2} & 0 & 0 & 0 \\
0 & \sigma_{2} & 0 & 0 \\
0 & 0 & -\sigma_{2} & 0 \\
0 & 0 & 0 & -\sigma_{2}
\end{array}\right), \quad F_{(2)}=\frac{i}{2}\left(\begin{array}{cccc}
\sigma_{2} & 0 & 0 & 0 \\
0 & -\sigma_{2} & 0 & 0 \\
0 & 0 & \sigma_{2} & 0 \\
0 & 0 & 0 & -\sigma_{2}
\end{array}\right), \\
F_{(3)} & =\frac{i}{2}\left(\begin{array}{cccc}
\sigma_{2} & 0 & 0 & 0 \\
0 & -\sigma_{2} & 0 & 0 \\
0 & 0 & -\sigma_{2} & 0 \\
0 & 0 & 0 & \sigma_{2}
\end{array}\right) .
\end{aligned}
$$

These flavor currents are thus $j_{(\alpha)}^{\mu} \equiv F_{(\alpha)}^{I J} j_{I J}^{\mu}$. They are normalized so that

$$
\left\langle j_{(\alpha)}^{\mu}(\vec{x}) j_{(\beta)}^{\nu}(0)\right\rangle=\frac{c_{T}}{16} P^{\mu \nu} \frac{\delta_{\alpha \beta}}{16 \pi^{2}|\vec{x}|^{2}} .
$$

The dimension- 1 scalars that are part of $\mathbf{3 5}_{v}$ and that belong to the same $\mathcal{N}=2$ multiplet as these flavor currents in $(2.15)$ are $J_{(\alpha)}=M_{(\alpha)}^{I J} \mathcal{O}_{I J}$, where

$$
\begin{aligned}
& M_{(1)}=\frac{1}{4} \operatorname{diag}\{1,1,1,1,-1,-1,-1,-1\}, \\
& M_{(2)}=\frac{1}{4} \operatorname{diag}\{1,1,-1,-1,1,1,-1,-1\}, \\
& M_{(3)}=\frac{1}{4} \operatorname{diag}\{1,1,-1,-1,-1,-1,1,1\},
\end{aligned}
$$

respectively. From (2.12), we have

$$
\left\langle J_{(\alpha)}(\vec{x}) J_{(\beta)}(0)\right\rangle=\frac{c_{2}}{2|\vec{x}|^{2}} \delta_{\alpha \beta} .
$$

Comparing (2.16) and (2.18) to (2.4), we see that the real scalars $J_{(\alpha)}$ are canonically normalized in the sense of (2.4) provided that

$$
c_{2}=\frac{c_{T}}{8(4 \pi)^{2}}, \quad \tau=\frac{c_{T}}{16} .
$$

In order to find $c_{3}$ by using (2.8), we should identify linear combinations of the $\mathbf{3 5}_{v}$ operators that reduce to chiral and anti-chiral operators from an $\mathcal{N}=2$ point of view. It can be checked that

$$
\mathcal{O}=\mathcal{O}_{11}-\mathcal{O}_{22}+2 i \mathcal{O}_{12}, \quad \overline{\mathcal{O}}=\mathcal{O}_{11}-\mathcal{O}_{22}-2 i \mathcal{O}_{12}
$$


are such operators because they have R-charges 1 and -1 under (2.14). From (2.15), we see that they have flavor charges 1 and -1 , respectively, under each of the currents $j_{(\alpha)}^{\mu}$. From (2.13), we have

$$
\frac{\left.\left\langle\mathcal{O}\left(\vec{x}_{1}\right) \overline{\mathcal{O}}\left(\vec{x}_{2}\right) J_{(\alpha)}\left(\vec{x}_{3}\right)\right\rangle\right\rangle^{2}}{\left\langle\mathcal{O}\left(\vec{x}_{1}\right) \overline{\mathcal{O}}\left(\vec{x}_{3}\right)\right\rangle\left\langle\mathcal{O}\left(\vec{x}_{3}\right) \overline{\mathcal{O}}\left(\vec{x}_{2}\right)\right\rangle\left\langle J_{(\alpha)}\left(\vec{x}_{1}\right) J_{(\alpha)}\left(\vec{x}_{2}\right)\right\rangle}=\frac{c_{3}^{2}}{2 c_{2}^{3}} .
$$

Identifying $q=1$ and using $\tau=c_{T} / 16$ as in (2.19), we have from (2.8) that

$$
\frac{\left.\left\langle\mathcal{O}\left(\vec{x}_{1}\right) \overline{\mathcal{O}}\left(\vec{x}_{2}\right) J_{(\alpha)}\left(\vec{x}_{3}\right)\right\rangle\right\rangle^{2}}{\left\langle\mathcal{O}(\vec{x}) \overline{\mathcal{O}}\left(\vec{x}_{2}\right)\right\rangle\left\langle\mathcal{O}\left(\vec{x}_{3}\right) \overline{\mathcal{O}}\left(\vec{x}_{2}\right)\right\rangle\left\langle J_{(\alpha)}\left(\vec{x}_{1}\right) J_{(\alpha)}\left(\vec{x}_{2}\right)\right\rangle}=\frac{16}{c_{T}} .
$$

A comparison of (2.21) and (2.22) gives

$$
\frac{c_{3}^{2}}{c_{2}^{3}}=\frac{32}{c_{T}}
$$

For canonically normalized $\mathcal{O}_{I J}$ for which $c_{2}$ is given by $(2.19)$, we have

$$
c_{3}=\frac{c_{T}}{4} \frac{1}{(4 \pi)^{3}} \text {. }
$$

\subsubsection{Summary}

To summarize, the 2- and 3-point functions of the canonically normalized $\mathbf{3 5}_{v}$ operators in an $\mathcal{N}=8$ SCFT are

$$
\begin{aligned}
\left\langle\mathcal{O}\left(\vec{x}_{1}, M_{1}\right) \mathcal{O}\left(\vec{x}_{2}, M_{2}\right)\right\rangle & =\frac{c_{T}}{8} \frac{1}{(4 \pi)^{2}} \frac{\operatorname{tr}\left(M_{1} M_{2}\right)}{\left|\vec{x}-\vec{x}_{2}\right|^{2}}, \\
\left\langle\mathcal{O}\left(\vec{x}_{1}, M_{1}\right) \mathcal{O}\left(\vec{x}_{2}, M_{2}\right) \mathcal{O}\left(\vec{x}_{3}, M_{3}\right)\right\rangle & =\frac{c_{T}}{4} \frac{1}{(4 \pi)^{3}} \frac{\operatorname{tr}\left(M_{1} M_{2} M_{3}+M_{1} M_{3} M_{2}\right)}{\left|\vec{x}_{1}-\vec{x}_{2}\right|\left|\vec{x}_{1}-\vec{x}_{3}\right|\left|\vec{x}_{2}-\vec{x}_{3}\right|},
\end{aligned}
$$

where $c_{T}$ is defined in (2.9).

In general, the quantity, $c_{T}$, depends on the parameters and dynamics of the (S)CFT in question. For an (S)CFT with a holographic dual, $c_{T}$ is a simple universal function of $L$ and $G_{4}$-it must be universal because the correlator $\left\langle T_{\mu \nu}(\vec{x}) T_{\rho \sigma}(0)\right\rangle$ is unique and depends only on $L$ and $G_{4}$. In the rest of this paper, we will be interested in theories with $\mathrm{AdS}_{4}$ duals. If $L$ is the radius of $\mathrm{AdS}_{4}$ and $G_{4}$ is the effective Newton constant in four-dimensions, we have [47]:

$$
c_{T}=\frac{32 L^{2}}{\pi G_{4}} .
$$

The correlation functions (2.25) then become:

$$
\begin{aligned}
\left\langle\mathcal{O}\left(\vec{x}_{1}, M_{1}\right) \mathcal{O}\left(\vec{x}_{2}, M_{2}\right)\right\rangle & =\frac{L^{2}}{4 \pi^{3} G_{4}} \frac{\operatorname{tr}\left(M_{1} M_{2}\right)}{\left|\vec{x}_{1}-\vec{x}_{2}\right|^{2}}, \\
\left\langle\mathcal{O}\left(\vec{x}_{1}, M_{1}\right) \mathcal{O}\left(\vec{x}_{2}, M_{2}\right) \mathcal{O}\left(\vec{x}_{3}, M_{3}\right)\right\rangle & =\frac{L^{2}}{8 \pi^{4} G_{4}} \frac{\operatorname{tr}\left(M_{1} M_{2} M_{3}+M_{1} M_{3} M_{2}\right)}{\left|\vec{x}_{1}-\vec{x}_{2}\right|\left|\vec{x}_{1}-\vec{x}_{3}\right|\left|\vec{x}_{2}-\vec{x}_{3}\right|} .
\end{aligned}
$$

One of our main goals in the remainder of this paper is to reproduce these formulas from a holographic computation. 


\subsubsection{An example}

Ref. [14] considered only three of the 35 operators, denoted $\mathcal{O}_{\alpha}$, with $\alpha=1,2,3$, corresponding to

$$
\mathcal{O}_{\alpha}=2 J_{(\alpha)}
$$

with $J_{(\alpha)}$ defined right above (2.17). The 2-point function of $\mathcal{O}_{\alpha}$ is

$$
\left\langle\mathcal{O}_{\alpha}\left(\vec{x}_{1}\right) \mathcal{O}_{\beta}\left(\vec{x}_{2}\right)\right\rangle=\frac{L^{2}}{2 \pi^{3} G_{4}} \frac{\delta_{\alpha \beta}}{\left|\vec{x}_{1}-\vec{x}_{2}\right|^{2}} .
$$

Using (2.27), one can check that all 3-point functions between $\mathcal{O}_{\alpha}$ vanish except for

$$
\left\langle\mathcal{O}_{1}\left(\vec{x}_{1}\right) \mathcal{O}_{2}\left(\vec{x}_{2}\right) \mathcal{O}_{3}\left(\vec{x}_{3}\right)\right\rangle=\frac{L^{2}}{4 \pi^{4} G_{4}} \frac{1}{\left|\vec{x}_{1}-\vec{x}_{2}\right|\left|\vec{x}_{1}-\vec{x}_{3}\right|\left|\vec{x}_{2}-\vec{x}_{3}\right|},
$$

as well as symmetric permutations of $\mathcal{O}_{\alpha}$. For a different computation of these correlation functions, see appendix A. ${ }^{8}$

\section{Boundary terms in $\mathcal{N}=1$ truncations}

\subsection{Review of the Bogomolny argument in [14]}

The first (not so gentle) hint that a boundary counterterm may provide the answer to the puzzle of the vanishing $\left\langle\mathcal{O}_{1}\left(\vec{x}_{1}\right) \mathcal{O}_{2}\left(\vec{x}_{2}\right) \mathcal{O}_{3}\left(\vec{x}_{3}\right)\right\rangle$ correlator from bulk supergravity came from appendix $\mathrm{C}$ of [14]. In this reference, a Bogomolny argument was used to generate the BPS equations for a general $\mathcal{N}=1$ supergravity model with asymptotically $\mathrm{AdS}_{4}$ solution. The model contains chiral multiplets with a Kähler target space with Kähler potential $K(z, \bar{z})$ and a holomorphic superpotential $W_{\mathrm{SG}}(z)$. We now summarize the results.

When the domain wall Ansatz

$$
d s^{2}=e^{2 \mathcal{A}(r)} \eta_{a b} d x^{a} d x^{b}+d r^{2}, \quad z^{\alpha}=z^{\alpha}(r), \quad \bar{z}^{\bar{\beta}}=\bar{z}^{\bar{\beta}}(r)
$$

is inserted in the (Lorentzian signature) bosonic action

$$
\begin{aligned}
S & =\frac{1}{8 \pi G_{4}} \int d^{4} x \sqrt{-g}\left[\frac{1}{2} R-K_{\alpha \bar{\beta}} \partial_{\mu} z^{\alpha} \partial^{\mu} \bar{z}^{\bar{\beta}}-V_{\mathrm{SG}}\right], \\
V_{\mathrm{SG}} & =e^{K}\left[g^{\alpha \bar{\beta}} \nabla_{\alpha} W_{\mathrm{SG}} \nabla_{\bar{\beta}} \bar{W}_{\mathrm{SG}}-3 W_{\mathrm{SG}} \bar{W}_{\mathrm{SG}}\right], \\
\nabla_{\alpha} W_{\mathrm{SG}} & =\left(\partial_{\alpha}+K,_{\alpha}\right) W_{\mathrm{SG}}, \quad \nabla_{\bar{\beta}} \bar{W}_{\mathrm{SG}}=\left(\partial_{\bar{\beta}}+K, \bar{\beta}\right) \bar{W}_{\mathrm{SG}},
\end{aligned}
$$

the action can be manipulated by partial integration and turned into a sum of quadratic factors which are the BPS equations

$$
\begin{aligned}
& \partial_{r} z^{\alpha}=-e^{K / 2} \sqrt{W_{\mathrm{SG}} / \bar{W}_{\mathrm{SG}}} K^{\alpha \bar{\gamma}} \nabla_{\bar{\gamma}} \bar{W}_{\mathrm{SG}}, \\
& \partial_{r} \bar{z}^{\bar{\beta}}=-e^{K / 2} \sqrt{\bar{W}_{\mathrm{SG}} / W_{\mathrm{SG}}} K^{\delta \bar{\beta}} \nabla_{\delta} W_{\mathrm{SG}}, \\
& \partial_{r} \mathcal{A}=e^{K / 2}\left|W_{\mathrm{SG}}\right|,
\end{aligned}
$$

\footnotetext{
${ }^{8}$ It should also be possible to calculate $c_{2}$ and $c_{3}$ directly in the SCFT using the gauged quantum mechanics obtained in [48].
} 
plus the boundary term (at the cutoff $r_{0}$ )

$$
S_{\text {cutoff }}=\frac{1}{4 \pi G_{4}} \int d^{3} x d r \frac{\partial}{\partial r}\left(\sqrt{-g} e^{K / 2}\left|W_{\mathrm{SG}}\right|\right)=\frac{1}{4 \pi G_{4}} \int d^{3} x e^{3 \mathcal{A}} e^{K / 2}\left|W_{\mathrm{SG}}\right| .
$$

This surface term must be cancelled by adding an equal and opposite counterterm to the action, which we will do momentarily.

In the specific 3-scalar truncation studied in [14], the superpotential and Kähler potential are

$$
W_{\mathrm{SG}}=\frac{1+z^{1} z^{2} z^{3}}{L}, \quad K=-\sum_{\alpha=1}^{3} \log \left[1-\left|z^{\alpha}\right|^{2}\right] .
$$

The constant term in $W_{\mathrm{SG}}$ determines AdS scale. The warp factor of the domain wall solution tends to $e^{2 \mathcal{A}(r)} \rightarrow e^{2 r / L}$ at large $r$, and the scalars vanish at the rate $z^{\alpha}(r) \sim e^{-r / L}$. The counterterm, which is Kähler invariant, is (at fixed large $r$ )

$$
\begin{aligned}
& S_{\mathrm{BPS}}=-\frac{1}{4 \pi G_{4}} \int d^{3} x e^{3 A} e^{K / 2} \mid W_{\mathrm{SG}} \mid \\
&=-\frac{1}{4 \pi G_{4} L} \int d^{3} x e^{3 r / L}\left[\begin{array}{rl}
1 & +\frac{1}{2} \delta_{\alpha \bar{\beta}} z^{\alpha}(r) \bar{z}^{\bar{\beta}}(r) \\
& \left.+\frac{1}{2}\left(z^{1}(r) z^{2}(r) z^{3}(r)+\text { c.c. }\right)+\ldots\right] .
\end{array}\right.
\end{aligned}
$$

The constant part of $\left|W_{\mathrm{SG}}\right|$ gives a cubic divergence as $r \rightarrow \infty$, and the quadratic term from the Kähler potential gives a linear divergence. Both terms agree with standard counterterms from holographic renormalization. The third term is finite, and it is this that provides the boundary cubic vertex which will be used to calculate $\left\langle\mathcal{O}_{1}\left(\vec{x}_{1}\right) \mathcal{O}_{2}\left(\vec{x}_{2}\right) \mathcal{O}_{3}\left(\vec{x}_{3}\right)\right\rangle$ in section 8.2.

It is important to point out that the precise agreement of the free energy found in [14] between the AdS/CFT result and that from supersymmetric localization in the dual ABJM field depended crucially on the added cubic counterterm. Since BPS domain walls are supersymmetric, the new counterterm is a consequence of SUSY.

\subsection{Boundary terms required by supersymmetry}

In most studies of supergravity theories, boundary terms generated in the process of checking local supersymmetry are discarded, since the supersymmetry parameters, $\epsilon(r, \vec{x})$, are arbitrary functions and may be assumed to vanish rapidly at the boundary. However, in AdS, the spinors $\epsilon(r, \vec{x})$, are required to approach an AdS Killing spinor at the boundary and this leads to finite and even divergent boundary contributions. Without the addition of appropriate boundary terms, as we will explain, the action is simply not supersymmetric.

Let us be more precise. The most basic AdS/CFT setup involves the study of the states in a CFT. This is to be contrasted with the study of relevant deformations and correlation functions of the CFT via holographic sources, which we will discuss in the next paragraph. In general, the states of a CFT are described by bulk field configurations obeying boundary conditions that 1) provide a well-defined Euler-Lagrange principle, namely 
that the Euler-Lagrange equations follow from the vanishing of the variation of the action, without discarding any boundary terms. If the CFT is supersymmetric, which is the case of interest here, the boundary conditions used to describe states of the CFT must also 2) be preserved under arbitrary supersymmetry variations; and 3) ensure that the action is supersymmetric, also without discarding any boundary terms. The point we will make is that these conditions cannot be obeyed without the addition of certain boundary counterterms. See also [23, 24, 27-31, 31, 49-59].

As a more involved application of AdS/CFT to supersymmetric field theories, one generalizes the boundary conditions discussed above to allow for deformations of the CFT by introducing sources for relevant operators. For a general given source configuration, the action will not be supersymmetric. ${ }^{9}$ Instead, supersymmetry relates various source configurations to one another. So, the on-shell supergravity action, when viewed as a functional of the various field theory sources, should still be supersymmetric, provided that the sources are transformed appropriately instead of being held fixed. Indeed, it is usually the on-shell action $S_{\text {on-shell }}$, viewed as a functional of various field theory sources, that is interpreted by the AdS/CFT dictionary as the generating functional of connected correlation functions, and this generating functional should be supersymmetric. We will actually deal with a somewhat exceptional application of $A d S / C F T$, because the three bulk scalars $A^{\alpha}=\operatorname{Re} z^{\alpha}$ in the $\mathcal{N}=1$ truncation (and the $35 \alpha^{i j k l}$ in the $\mathcal{N}=8$ theory) are dual to $\Delta=1$ operators in the dual CFT. In this case it is not $S_{\text {on-shell }}$ but rather its Legendre transform [25], defined and called $\tilde{S}_{\text {on-shell }}$ in section 3.6 below, that is the generating functional. Supersymmetry requires that this generating functional is supersymmetric, provided that the field theory sources are assigned appropriate transformation rules. ${ }^{10}$

In the remainder of this section, we determine the boundary counterterms that ensure that $\tilde{S}_{\text {on-shell }}$ is supersymmetric. In the limit in which the cutoff is removed, $r_{0} \rightarrow \infty$, we find a set of infinite and finite boundary counterterms. The infinite counterterms agree with those obtained by holographic renormalization and the finite ones include the finite term of $S_{\mathrm{BPS}}$ in (3.6).

Since gauged $\mathcal{N}=8$ supergravity is a rather complicated theory, we first present a detailed illustration of the technique in a far simpler model, an $\mathcal{N}=1$ model with global SUSY in $\mathrm{AdS}_{4}$. This model is obtained in a limit of $\mathcal{N}=1$ supergravity, similar to that of [36], in which the back-reaction of matter fields on the spacetime geometry is consistently suppressed. We then outline the extension of the method to $\mathcal{N}=1$ supergravity and finally proceed to derive the analogous results in the $\mathcal{N}=8$ theory.

\subsection{The global limit of $\mathcal{N}=1, \mathrm{AdS}_{4}$ supergravity}

In this section we derive the action and transformation rules of chiral multiplets of a global SUSY model on a fixed $\mathrm{AdS}_{4}$ background geometry. We derive this model from $\mathcal{N}=1$

\footnotetext{
${ }^{9}$ For certain special source configurations, the action may preserve a fraction of the supersymmetries preserved by the vacuum.

${ }^{10}$ Alternate quantization and the Legendre transform are needed to describe CFT operators whose scale dimension is given by the lower sign in the AdS/CFT mass fomula $\Delta=\left(d \pm \sqrt{d^{2}+4 m^{2} L^{2}}\right) / 2$.
} 
supergravity written in conventions very similar ${ }^{11}$ to those of chapter 18 of [60]. The action is normalized as ${ }^{12,13}$

$$
\begin{aligned}
S_{\mathrm{SG}}=\int d^{4} x & \sqrt{-g}\left[\frac{1}{2 \kappa^{2}}\left(R-\bar{\psi}_{\mu} \gamma^{\mu \nu \rho} \nabla_{\nu} \psi_{\rho}\right)\right. \\
& \left.-g_{\alpha \bar{\beta}}\left(\partial_{\mu} z^{\alpha} \partial^{\mu} \bar{z}^{\bar{\beta}}+\frac{1}{2} \bar{\chi}^{\alpha} \not P_{R} \chi^{\bar{\beta}}+\frac{1}{2} \bar{\chi}^{\bar{\beta}} \not P_{L} \chi^{\alpha}\right)+\cdots\right] .
\end{aligned}
$$

Factors of $\kappa$ with $\kappa^{2}=8 \pi G_{4}$ are included in the non-linear terms indicated by $\cdots$.

The dynamics of the supergravity model is specified by a Kähler potential $K(z, \bar{z})$ and a holomorphic superpotential of the form

$$
W_{\mathrm{SG}}(z)=\frac{1}{\kappa^{2} L}+W(z) \longrightarrow \frac{1}{\kappa^{2} L}+\frac{\kappa}{L} z^{1} z^{2} z^{3} .
$$

The superpotential in the $\mathcal{N}=1$ truncation of $\mathcal{N}=8$ supergravity studied in [14] appears on the right. The condition that the theory admit a supersymmetric $\mathrm{AdS}_{4}$ solution of scale $L$ is that

$$
\nabla_{\alpha} W_{\mathrm{SG}} \equiv\left(\partial_{\alpha}+\kappa^{2} \partial_{\alpha} K\right) W_{\mathrm{SG}}=0,
$$

is satisfied at $z_{\alpha}=0$. This condition is fulfilled in the model of [14].

The global limit of the supergravity action is obtained via the following procedure:

1. Fix the $\mathrm{AdS}_{4}$ background and use coordinates $r, x^{a}, a=0,1,2$ in which

$$
d s^{2}=e^{2 r / L} \eta_{a b} d x^{a} d x^{b}+d r^{2} .
$$

2. Set the gravitino field to $\psi_{\mu}=0$. This is consistent if we require that the SUSY parameters are Killing spinors of $\mathrm{AdS}_{4}$ and thus satisfy

$$
\nabla_{\mu} \epsilon=-\frac{1}{2 L} \gamma_{\mu} \epsilon \quad \Longrightarrow \quad \not \epsilon=-\frac{2}{L} \epsilon
$$

3. Use (3.8) to obtain the superpotential $W(z)$ of the global model.

4. Keep the $\kappa$ factors in $K(z, \bar{z})$ and $W(z)$, but otherwise drop all terms in the supergravity action with positive powers of $\kappa$.

When this procedure is applied to the scalar potential of $\mathcal{N}=1$ supergravity, one obtains

$$
\begin{aligned}
V_{\mathrm{SG}} & \equiv e^{\kappa^{2} K}\left[g^{\alpha \bar{\beta}} \nabla_{\alpha} W_{\mathrm{SG}} \nabla_{\bar{\beta}} \bar{W}_{\mathrm{SG}}-3 \kappa^{2} W_{\mathrm{SG}} \bar{W}_{\mathrm{SG}}\right] \\
& =g^{\alpha \bar{\beta}}\left(\partial_{\alpha} W(z)+\frac{1}{L} \partial_{\alpha} K\right)\left(\partial_{\bar{\beta}} \bar{W}(\bar{z})+\frac{1}{L} \partial_{\bar{\beta}} K\right)-\frac{3}{L}(W+\bar{W})-3 K+O\left(\kappa^{2}\right),
\end{aligned}
$$

\footnotetext{
${ }^{11}$ Here we scale the SUSY parameter $\epsilon$ of $[60]$ to $\sqrt{2} \epsilon$.

${ }^{12}$ To avoid potential confusion, we note that complex scalars in this section are canonical and have engineering dimension 1 . They are related to the dimensionless scalars of [14] and previous sections of this paper by $z_{\text {here }}=z_{\text {there }} / \kappa$. When this and the analogous scaling is made for spinors, the supergravity action acquires the overall factor $1 / 8 \pi G_{4}$.

${ }^{13}$ For clarity, we write $\not \nabla=\gamma^{\mu} \partial_{\mu}$ as an operator acting on the $4 \mathrm{~d}$ fields, and $\not \partial=\gamma^{a} \partial_{a}$ as an operator acting only in the boundary directions.
} 
which agrees with (3.8) of [36]. An additional cosmological constant term $-3 / \kappa^{2} L^{2}$ has been dropped since it is part of the gravitational sector whose solution is fixed.

The entire action obtained from our procedure agrees with (3.5) of [36]. However, we now make a further assumption which simplifies the analysis needed for our main purpose which is to determine the boundary terms in the variation of the action. Namely, we assume that the Kähler metric is flat. This is justified because the Kähler potential of the $\mathcal{N}=1$ truncation and the parent $\mathcal{N}=8$ theory has the structure

$$
K(z, \bar{z})=z \bar{z}+a_{2}(z \bar{z})^{2}+a_{3}(z \bar{z})^{3}+\cdots .
$$

In models with cubic $W(z)$, scalar masses $m^{2}=-2 / L^{2}$ are entirely determined by the conformal coupling, so the leading asymptotic behavior of scalar fields is $z(r, x) \sim e^{-r / L}$. Thus the effects of target space curvature are suppressed by $e^{-2 r / L}$ relative to the leading term, and they play no role in the determination of boundary terms.

After the procedure above is implemented we make the further step of introducing auxiliary $F, \bar{F}$ fields. It is also sufficient to consider a single chiral mutiplet $\left(z, P_{L} \chi, F\right)$. This enables us to write the action as ${ }^{14}$

$$
S_{\mathrm{bulk}}=S_{\mathrm{kin}}+S_{F}+S_{\bar{F}},
$$

where

$$
\begin{aligned}
& S_{\text {kin }}=\int d^{4} x \sqrt{-g}\left[-\partial_{\mu} z \partial^{\mu} \bar{z}-\frac{1}{2}\left(\bar{\chi} \not P_{L} \chi+\bar{\chi} \not \vec{P} P_{R} \chi\right)\right. \\
& \left.+\left(F+\frac{z}{L}\right)\left(\bar{F}+\frac{\bar{z}}{L}\right)+\frac{2}{L^{2}} z \bar{z}\right] \\
& S_{F}=\int d^{4} x \sqrt{-g}\left[F W^{\prime}-\frac{1}{2} W^{\prime \prime} \bar{\chi} P_{L} \chi+\frac{3}{L} W\right], \quad S_{\bar{F}}=\left(S_{F}\right)^{\dagger} .
\end{aligned}
$$

It is very useful to have three terms which are separately invariant under the transformation rules:

$$
\begin{array}{lll}
\delta z=\bar{\epsilon} P_{L} \chi, & \delta P_{L} \chi=P_{L}(\not \nabla z+F) \epsilon, & \delta F=\bar{\epsilon}(\not \nabla-1 / L) P_{L} \chi, \\
\delta \bar{z}=\bar{\epsilon} P_{R} \chi, & \delta P_{R} \chi=P_{R}(\not \nabla \bar{z}+\bar{F}) \epsilon, & \delta \bar{F}=\bar{\epsilon}(\not \nabla-1 / L) P_{R} \chi .
\end{array}
$$

The proof of invariance is quite simple for $S_{F}$ :

$$
\begin{aligned}
\delta S_{F}=\int d^{4} x \sqrt{-g}[ & F W^{\prime \prime} \bar{\epsilon} P_{L} \chi-W^{\prime \prime} \bar{\epsilon}(-\not z z+F) P_{L} \chi \\
& \left.+W^{\prime} \bar{\epsilon}\left(\not \nabla-\frac{1}{L}\right) P_{L} \chi+\frac{3}{L} W^{\prime}\left(\bar{\epsilon} P_{L} \chi\right)-W^{\prime \prime \prime}\left(\bar{\epsilon} P_{L} \chi\right)\left(\bar{\chi} P_{L} \chi\right)\right] .
\end{aligned}
$$

Terms involving $F$ cancel and the $W^{\prime \prime \prime}$ term vanishes by Fierz rearrangement. The remaining terms can be written as

$$
\begin{aligned}
\delta S_{F} & =\int d^{4} x \sqrt{-g}\left[\bar{\epsilon} \not \nabla\left(W^{\prime} P_{L} \chi\right)+\frac{2}{L} W^{\prime} \bar{\epsilon} P_{L} \chi\right] \\
& =\int d^{4} x \sqrt{-g}\left[\nabla_{\mu}\left(W^{\prime} \bar{\epsilon} \gamma^{\mu} P_{L} \chi\right)-\bar{\epsilon}\left(\overleftarrow{\not}-\frac{2}{L}\right) W^{\prime} P_{L} \chi\right]
\end{aligned}
$$

\footnotetext{
${ }^{14}$ This action was studied in section 3 of [61].
} 
The last term vanishes by the (adjoint of the) Killing spinor equation (3.11), and the first term is the total derivative which is the goal of the calculation.

It is more difficult to show that $\delta S_{\text {kin }}$ is invariant up to boundary terms. Details are given in appendix B. Here we simply write the final expression that contains the residual boundary terms

$$
\delta S_{\text {kin }}=\frac{1}{2} \int d^{4} x \sqrt{-g} \nabla_{\mu}\left[\bar{\epsilon} \gamma^{\mu}\left(-\not \nabla\left(z P_{R}+\bar{z} P_{L}\right)+\frac{2}{L}\left(z P_{R}+\bar{z} P_{L}\right)+\left(F P_{R}+\bar{F} P_{L}\right)\right) \chi\right] .
$$

The analysis above is valid for a general superpotential, $W(z)$. However, we are specifically concerned with a cubic $W(z)$, which, for the purpose of providing a toy model, we take to be

$$
W(z)=\frac{\kappa z^{3}}{3 L} .
$$

The consistent truncation of $\mathcal{N}=8$ studied in [62-64] contains three identical chiral multiplets and is trivially related to ours, as is the truncation to three chiral multiplets with $W=\kappa z^{1} z^{2} z^{3} / L$ of $[14]$.

Finally we note that auxiliary fields are eliminated and real fields are introduced using

$$
F=-\frac{z}{L}-\bar{W}^{\prime}=-\frac{z}{L}-\kappa \frac{\bar{z}^{2}}{L^{2}}, \quad z=A+i B
$$

\subsection{Further conventions and asymptotic behavior}

Before determining the boundary counterterms that ensure supersymmetry it is useful to state our conventions more completely and to discuss the asymptotic behavior of the various actors in our drama.

In the natural Lorentz frame, $e^{a}=e^{r / L} d x^{a}$ and $e^{3}=d r, a=0,1,2$, for the metric (3.10), $\left(\gamma^{a}, \gamma^{3}\right)$ are constant $\gamma$-matrices for signature $(-+++)$. As usual, the $\gamma$-matrices with a Greek index are defined by $\gamma^{\mu}=e_{a}{ }^{\mu} \gamma^{a}+e_{3}{ }^{\mu} \gamma^{3}$. In the language of the Cartan structure equations, the connection 1-forms are $\omega^{a 3}=e^{a} / L, \omega^{a b}=0$.

The Killing spinors of the Poincaré patch are Majorana spinors. In $\mathrm{AdS}_{4}$, the Killing spinor equation (3.11) has solutions of the form

$$
\epsilon=e^{r / 2 L} \eta_{-}+e^{-r / 2 L} \eta_{+},
$$

with coefficients $\eta_{-}(\vec{x})$ and $\eta_{+}(\vec{x})$ that obey $\not \partial \eta_{+}=0$ and $\not \partial \eta_{-}=-(3 / L) \eta_{+}$and have definite "radiality":

$$
\gamma^{3} \eta_{ \pm}= \pm \eta_{ \pm}, \quad \bar{\eta}_{ \pm} \gamma^{3}=\mp \bar{\eta}_{ \pm}
$$

In particular, there are two linearly independent Poincaré supersymmetries that have $\eta_{+}=0$ and $\eta_{-}=$constant, as well as two superconformal supersymmetries that have $\eta_{+}=$constant and $\eta_{-}=-\gamma_{a} x^{a} \eta_{+} / L .^{15}$

\footnotetext{
${ }^{15}$ The designations Poincaré and superconformal arise because the associated supercharges anti-commute to translations and, respectively, special conformal transformations of the isometry group $\mathrm{SO}(3,2)$.
} 
The behavior of solutions of the field equations as $r \rightarrow \infty$ is (with $z=A+i B$ )

$$
\begin{aligned}
& A(r, \vec{x})=e^{-r / L} A_{1}(\vec{x})+e^{-2 r / L} A_{2}(\vec{x})+\ldots, \\
& B(r, \vec{x})=e^{-r / L} B_{1}(\vec{x})+e^{-2 r / L} B_{2}(\vec{x})+\ldots, \\
& \chi(r, \vec{x})=e^{-3 r / 2 L} \chi_{3 / 2}(\vec{x})+e^{-5 r / 2 L} \chi_{5 / 2}(\vec{x})+\ldots
\end{aligned}
$$

The leading rates are standard in $\mathrm{AdS}_{4} / \mathrm{CFT}_{3}$ for scalars of mass $m^{2}=-2 / L^{2}$ and massless spinors. In a free theory, i.e. $W(z)=0$, the asymptotic series for $A$ and $B$ would contain exponential rates $e^{-k r / L}$ with $k$ either even or odd [22]. The presence of mixed even and odd integer rates occurs with interactions and is important in our analysis.

From the bulk supersymmetry variations (3.17) and the decomposition (3.23) for the Killing spinors, we find the supersymmetry transformations of the various coefficients appearing in the boundary expansion (3.25):

$$
\begin{aligned}
\delta A_{1} & =\frac{1}{2} \bar{\eta}_{-} \chi_{3 / 2+}, \quad \delta A_{2}=\frac{1}{2}\left(\bar{\eta}_{-} \chi_{5 / 2+}+\bar{\eta}_{+} \chi_{3 / 2-}\right) \\
\delta B_{1} & =-\frac{i}{2} \bar{\eta}_{-} \gamma^{5} \chi_{3 / 2-}, \quad \delta B_{2}=-\frac{i}{2}\left(\bar{\eta}_{-} \gamma^{5} \chi_{5 / 2-}+\bar{\eta}_{+} \gamma^{5} \chi_{3 / 2+}\right), \\
\delta \chi_{3 / 2-} & =\left(\frac{1}{L} A_{2}-\frac{\kappa}{L}\left(A_{1}^{2}-B_{1}^{2}\right)+i \gamma^{5} \not \partial B_{1}\right) \eta_{-}-\frac{2 i}{L} B_{1} \gamma^{5} \eta_{+}, \\
\delta \chi_{3 / 2+} & =i \gamma^{5}\left(\frac{1}{L} B_{2}+\frac{2 \kappa}{L} A_{1} B_{1}\right) \eta_{-}+\not \partial A_{1} \eta_{-}-\frac{2}{L} A_{1} \eta_{+} .
\end{aligned}
$$

Here and in the rest of this section we find it convenient to split the coefficient functions $\chi_{k}(\vec{x})$ appearing in the expansion of $\chi(r, \vec{x})$ into components of even and odd radiality, denoted by an additional \pm subscript:

$$
\chi_{k}(\vec{x})=\chi_{k+}(\vec{x})+\chi_{k-}(\vec{x}), \quad \gamma^{3} \chi_{k \pm}= \pm \chi_{k \pm} .
$$

\subsection{Counterterms and CFT states}

We now turn to our goal of finding the appropriate boundary counterterms that ensure supersymmetry. As already mentioned, the appropriate requirement in its most general form is that the Legendre transform of $S_{\mathrm{bulk}}+S_{\mathrm{bdy}}$, seen as a functional of the boundary theory sources, is supersymmetric. As a particular simpler case that does not require a Legendre transform, we first study the case where the boundary sources vanish and find the boundary counterterms $S_{\text {bdy }}$ that ensure supersymmetry, as explained in section 3.2. The counterterms $S_{\mathrm{bdy}}$ are initially evaluated at the cutoff $r=r_{0}$; in the limit $r_{0} \rightarrow \infty$ they are expressed in terms of the asymptotic coefficients of (3.25).

In determining the boundary conditions and boundary counterterms that ensure supersymmetry, we can take guidance from the fact that the pseudoscalar $B(r, \vec{x})$ is dual to a dimension 2 operator in the dual CFT. Consequently, the standard AdS/CFT dictionary identifies $B_{1}(\vec{x})$ as the field theory source for this operator. The condition of vanishing sources should therefore include $B_{1}(\vec{x})=0$. The supersymmetry variations (3.26) then identify a consistent set of boundary conditions on the other fields. Indeed, by considering 
$\delta B_{1}(\vec{x})$, one also obtains $\chi_{3 / 2-}(\vec{x})=0$, and then from $\delta \chi_{3 / 2-}(\vec{x})=0$ one further obtains $A_{2}(\vec{x})-\kappa A_{1}^{2}(\vec{x})=0$. In summary, the conditions of vanishing sources are

$$
B_{1}(\vec{x})=0, \quad \chi_{3 / 2-}(\vec{x})=0, \quad A_{2}(\vec{x})-\kappa A_{1}^{2}(\vec{x})=0,
$$

and they represent our desired boundary conditions. ${ }^{16}$

The boundary counterterms are then determined by ensuring that the boundary conditions (3.28) are consistent with the Euler-Lagrange variational principle. Let us examine the scalar part of the action first. Integrating out the auxiliary fields and using the cubic superpotential (3.21), the scalar part of the bulk action becomes

$$
S_{\mathrm{bulk}}=\int d^{4} x \sqrt{-g}\left[-\partial_{\mu} z \partial^{\mu} \bar{z}+\frac{2}{L^{2}} z \bar{z}-\frac{\kappa^{2}}{L^{2}}(z \bar{z})^{2}\right] .
$$

The Euler-Lagrange variation of the action reads

$$
\delta S_{\mathrm{bulk}}=\int d^{4} x \sqrt{-g}\left[\delta z(\text { eom for } \bar{z})+\delta \bar{z}(\text { eom for } z)-\nabla_{\mu}\left(\delta z \partial^{\mu} \bar{z}+\delta \bar{z} \partial^{\mu} z\right)\right] .
$$

The variational principle implies the equations of motion provided that we add a boundary term whose variation cancels the second term in (3.30). Using the asymptotic expansion (3.25) and the boundary conditions (3.28), we have

$$
\delta S_{\text {bdy }}+\frac{1}{L} \int d^{3} x\left[2 e^{r_{0} / L}\left(A_{1} \delta A_{1}\right)+8 \kappa A_{1}^{2} \delta A_{1}\right]=0
$$

where the second term in (3.31) comes from the last term in (3.30). From this expression we deduce that the required boundary term is

$$
S_{\mathrm{bdy}}=-\frac{1}{L} \int d^{3} x e^{3 r_{0} / L}\left[A^{2}+\frac{2 \kappa}{3} A^{3}\right]
$$

because its variation gives (3.31), again after using the boundary conditions (3.28). A similar analysis for the fermionic part of the action shows that there are no fermionic boundary terms that do not vanish under (3.28), the boundary term $S_{\text {bdy }}$ being the only boundary term that is needed. One can then check that the combined action $S_{\text {bulk }}+S_{\text {bdy }}$ is supersymmetric. This calculation is a particular case of the calculation performed in the next section, and we will defer it until then.

What we have done so far amounts to a "minimal supersymmetric completion" of the bulk action via the boundary term (3.32). Without this boundary term and the boundary conditions (3.28), the theory would not be supersymmetric.

\subsection{More general counterterms and the Legendre transform}

We now proceed to an analysis that is not restricted to the CFT states but allows nonvanishing sources for relevant operators. In particular, we relax the conditions (3.28) by

\footnotetext{
${ }^{16}$ It is well known [27-30] that one should choose one of the two asymptotic projections $\chi_{3 / 2 \pm}$ in (3.26) as the fermion source.
} 
allowing arbitrary field theory sources, as we will explain. We will determine a more general boundary action $S_{\text {bdy }}$ that reduces to (3.32) when the sources are taken to vanish as in (3.28).

Recall that the supersymmetry variation of the bulk action, $\delta S_{\text {bulk }}=\delta S_{\text {kin }}+\delta S_{F}+$ $\delta S_{\bar{F}}$, reduces to a boundary term given in (3.19)-(3.20). It is straightforward to cancel various contributions to $\delta S_{\text {bulk }}$ against the variation of an appropriately chosen boundary counterterm $S_{\mathrm{bdy}}$. For instance, it is clear that $\delta S_{F}$ (and its conjugate $\delta S_{\bar{F}}$ ) are finite at the boundary and can be nicely cancelled by the variation of the finite counterterm

$$
S_{3}=-\int d^{3} x e^{3 r_{0} / L}[W(z)+\bar{W}(\bar{z})] .
$$

The remaining boundary term $\delta S_{\text {kin }}$ is "linearly divergent." Its leading term grows as $e^{r_{0} / L}$ at the boundary when we include the factor $\sqrt{-h}=e^{3 r_{0} / L}, h$ being the determinant of the boundary metric. We expect that such divergences are cancelled by counterterms determined by holographic renormalization. The relevant counterterm can be obtained from (6.5) of [14]. With a sign change for Lorentzian signature and in the global limit and with current normalization, it is given by

$$
S_{2}=-\frac{1}{L} \int d^{3} x e^{3 r_{0} / L} \bar{z} z
$$

Upon adding $S_{2}$, the supersymmetry variation of the kinetic term (3.20) is finite at the boundary. After adjusting the normalization to that of section 3 and for cubic $W(z), S_{3}$ and $S_{2}$ agree perfectly with the cubic and quadratic terms of (3.6). In the rest of this section we will work with the cubic $W(z)=\kappa z^{3} / 3 L$ introduced in (3.21).

The remaining finite terms of $\delta\left(S_{\text {kin }}+S_{2}\right)$ (to be displayed in the next section) must still be cancelled, and two further modifications are needed. The first is to add another finite counterterm

$$
S_{\chi}=\frac{c}{4} \int d^{3} x e^{3 r_{0} / L} \bar{\chi} \chi
$$

This was proposed in the earliest papers on fermions in AdS/CFT [27-30] in order to obtain non-trivial 2-point correlators of fermionic operators in the boundary theory. The coefficient $c$ will be fixed at the value $c=1$ below. ${ }^{17}$

The second modification involves the Legendre transform that was mentioned in section 3.2. It is a more subtle issue that we now discuss in detail. We know that the scalar field $A$ is dual to a field theory operator of dimension 1, and hence obeys "alternate boundary conditions" as explained in [25]. Let us explain what this means by comparison to the pseudoscalar $B$, which is dual to a dimension-2 operator and obeys standard boundary conditions. For $B$, the leading coefficient in the boundary expansion $(3.25), B_{1}(\vec{x})$, is interpreted as a source for the dual operator. The Euler-Lagrange equations of motion are solved with the boundary condition of a prescribed value for $B_{1}(\vec{x})$, and the on-shell action is naturally thought of as a functional of $B_{1}(\vec{x})$. For $A$, it is not the leading coefficient,

\footnotetext{
${ }^{17}$ For a Dirac fermion, the coefficient was fixed in [29] and [30].
} 
$A_{1}(\vec{x})$, that should be interpreted as the source for the field theory operator, but instead its canonically conjugate quantity [25]

$$
\mathfrak{A}(\vec{x})=-\frac{\delta S_{\text {on-shell }}\left[A_{1}, \ldots\right]}{\delta A_{1}(\vec{x})} .
$$

Here, the ellipsis stands for other boundary data, such as $B_{1}(\vec{x})$, that can be interpreted as sources for field theory operators. The source $\mathfrak{A}(\vec{x})$ is sometimes loosely referred to as $A_{2}(\vec{x})$, because a simple calculation,

$$
\begin{aligned}
\mathfrak{A}(\vec{x})=-\lim _{r_{0} \rightarrow \infty} e^{-r_{0} / L} \frac{\delta S_{\text {on-shell }}}{\delta A\left(r_{0}, \vec{x}\right)} & =-\lim _{r_{0} \rightarrow \infty} e^{-r_{0} / L} \Pi_{A}\left(r_{0}, \vec{x}\right) \\
& =-\left(\frac{2}{L} A_{2}(\vec{x})-\frac{2 \kappa}{L}\left(A_{1}(\vec{x})^{2}-B_{1}(\vec{x})^{2}\right)\right),
\end{aligned}
$$

shows that, up to a normalization factor, it is equal to $A_{2}(\vec{x})$ plus non-linear corrections coming from the boundary terms (3.33)-(3.34). Note that $\mathfrak{A}(\vec{x})$ is the boundary limit of canonical momentum for the field $A(r, \vec{x})$, namely ${ }^{18}$

$$
\Pi_{A}(r, \vec{x})=e^{3 r / L} \frac{\partial \mathcal{L}}{\partial\left(\partial_{r} A(r, \vec{x})\right)}=-2 e^{3 r / L}\left(\partial_{r} A+\frac{1}{L} A+\frac{\kappa}{L}\left(A^{2}-B^{2}\right)\right),
$$

and that the second equality in (3.37) follows from the Hamilton-Jacobi equation.

The generating functional for connected correlators is the Legendre transform

$$
\tilde{S}_{\text {on-shell }}[\mathfrak{A}, \ldots]=S_{\text {on-shell }}\left[A_{1}, \ldots\right]+\int d^{3} x \mathfrak{A}(\vec{x}) A_{1}(\vec{x})
$$

evaluated after extremizing the r.h.s. with respect to $A_{1}(\vec{x})$. This extremization yields precisely (3.36). It is $\tilde{S}_{\text {on-shell }}$, and not $S_{\text {on-shell }}$, that is required to be supersymmetric when sources are present.

To ensure that $\tilde{S}_{\text {on-shell }}$ is supersymmetric, we need the supersymmetry variation $\delta \mathfrak{A}(\vec{x})$, and this must be chosen as the variation of (3.37) when the equations of motion are used. In particular, the fermion equation of motion implies

$$
\chi_{5 / 2+}=L \not \partial \chi_{3 / 2-}+2 \kappa\left(A_{1} \chi_{3 / 2+}+i B_{1} \gamma^{5} \chi_{3 / 2-}\right) .
$$

When combined with (3.37) and (3.26), this yields

$$
\delta \mathfrak{A}=-2 \delta\left(\frac{A_{2}}{L}-\frac{\kappa}{L}\left(A_{1}^{2}-B_{1}^{2}\right)\right)=-\left(\bar{\eta}_{-} \not \partial \chi_{3 / 2-}+\bar{\eta}_{+} \frac{1}{L} \chi_{3 / 2-}\right) .
$$

To summarize, we have added boundary terms to the bulk action of (3.14) to obtain the renormalized action

$$
S_{\mathrm{ren}}=S_{\mathrm{bulk}}+S_{\mathrm{bdy}}
$$

\footnotetext{
${ }^{18}$ Here $\mathcal{L}$ is the Lagrangian obtained from the action (3.14), augmented by conversion of the boundary actions (3.33)-(3.34) into total $\partial_{r}$ derivatives.
} 
where the bulk and boundary terms are

$$
S_{\text {bulk }} \equiv S_{\text {kin }}+S_{F}+S_{\bar{F}}, \quad S_{\text {bdy }} \equiv S_{2}+S_{\chi}+S_{3}
$$

The renormalized action $S_{\text {ren }}$ is denoted by $S_{\text {on-shell }}\left[A_{1}, \ldots\right]$ when equations of motion are satisfied. We identified the boundary limit $\mathfrak{A}(\vec{x})$ of the canonical momentum. We then defined the Legendre transform in (3.39) which a functional of $\mathfrak{A}$. This is the generating functional for correlation functions and will be used for this purpose in section 4 . In the next subsection we show that

$$
\delta\left(S_{\text {ren }}+S_{L}\right)=0, \quad S_{L} \equiv \int d^{3} x \mathfrak{A}(\vec{x}) A_{1}(\vec{x}),
$$

on-shell.

Before checking supersymmetry, let us make a comment about the field theory sources, which we have identified as $B_{1}, \chi_{3 / 2}$ and $\mathfrak{A}$. As argued in section 3.5, the three sources should then transform among themselves under SUSY. It is worth writing the SUSY variations of the sources that result from these assignments:

$$
\begin{aligned}
\delta B_{1} & =-\frac{i}{2} \bar{\eta}_{-} \gamma^{5} \chi_{3 / 2-}, \\
\delta \chi_{3 / 2-} & =\left(-i \not \partial B_{1} \gamma^{5}+\frac{1}{2} \mathfrak{A}\right) \eta_{-}-\frac{2 i}{L} B_{1} \gamma^{5} \eta_{+}, \\
\delta \mathfrak{A} & =-\left(\bar{\eta}_{-} \not \partial \chi_{3 / 2-}+\bar{\eta}_{+} \frac{1}{L} \chi_{3 / 2-}\right) .
\end{aligned}
$$

These transformations resemble the standard superconformal transformations of an $\mathcal{N}=1$, $d=3$ scalar multiplet, albeit with artefacts of their origin as the boundary limits of the bulk theory. It is straightforward to compute the commutator of two Poincaré supersymmetry transformations, those with $\eta_{+}=0$ and $\partial_{a} \eta_{-}=0$, as described below (3.24). In terms of the effectively two-component spinor parameters $\epsilon=i \gamma^{5} \eta_{-}$, the result is

$$
\left[\delta_{1}, \delta_{2}\right] \Phi(\vec{x})=-\left(\bar{\epsilon}_{1} \gamma^{a} \epsilon_{2}\right) \partial_{a} \Phi(\vec{x}),
$$

for all components $\Phi=B_{1}, \chi_{3 / 2-}, \mathfrak{A}$ of the multiplet. ${ }^{19}$

\subsection{Cancellation of the supersymmetry variation of the on-shell action}

Let us now show that (3.44) holds. We have already argued that

$$
\delta\left(S_{F}+S_{\bar{F}}+S_{3}\right)=0 .
$$

Our remaining task is to show that

$$
\delta\left(S_{\mathrm{kin}}+S_{2}+S_{\chi}+S_{L}\right)=0
$$

which we now proceed to do.

\footnotetext{
${ }^{19}$ We suggest that faithful readers try the Fierz rearrangement needed for the fermion.
} 
The variation of $S_{2}$ in (3.34) is

$$
\delta S_{2}=-\frac{1}{L} \int d^{3} x \sqrt{-g} \bar{\epsilon}\left(z P_{R}+\bar{z} P_{L}\right) \chi
$$

By adding it to $\delta S_{\text {kin }}$ in (3.20) we obtain

$$
\begin{array}{r}
\delta\left(S_{\text {kin }}+S_{2}\right)=\frac{1}{2} \int d^{3} x \sqrt{-g}\left[\bar{\epsilon}\left(-\gamma^{3} \not \partial+\frac{2}{L}\left(\gamma^{3}-I\right)\right)\left(z P_{R}+\bar{z} P_{L}\right) \chi\right. \\
\left.+\bar{\epsilon} \gamma^{3}\left(F P_{R}+\bar{F} P_{L}\right) \chi\right] .
\end{array}
$$

Using (3.22) as well as the boundary asymptotics (3.25), we obtain

$$
\begin{aligned}
\delta\left(S_{\text {kin }}+S_{2}\right)= & \frac{1}{2} \int d^{3} x\left[\bar{\eta}_{-}\left[\frac{A_{2}}{L}-\frac{\kappa}{L}\left(A_{1}^{2}-B_{1}^{2}\right)-\not \partial A_{1}\right] \chi_{3 / 2}\right. \\
& \left.+\bar{\eta}_{-}\left[-\frac{B_{2}}{L}-\frac{2 \kappa}{L} A_{1} B_{1}+\not \partial B_{1}\right] i \gamma^{5} \chi_{3 / 2}+\frac{1}{L} \bar{\eta}_{+}\left[-2 A_{1}+2 B_{1} i \gamma^{5}\right] \chi_{3 / 2}\right],
\end{aligned}
$$

where we took the limit $r_{0} \rightarrow \infty$.

Next, we have the variation $\delta S_{\chi}$ :

$$
\begin{aligned}
\delta S_{\chi}= & \frac{c}{2} \int d^{3} x\left[-\bar{\eta}_{-}\left[-\frac{1}{L}\left(A_{2}+i \gamma^{5} B_{2}\right)+\frac{\kappa}{L}\left(A_{1}^{2}-B_{1}^{2}-2 i A_{1} B_{1} \gamma^{5}\right)\right] \chi_{3 / 2}\right. \\
& \left.-\bar{\eta}_{-}\left[\not \partial A_{1}-i \gamma^{5} \not \partial B_{1}\right] \chi_{3 / 2}-\frac{1}{L} \bar{\eta}_{+}\left[2 A_{1}+2 i \gamma^{5} B_{1}\right] \chi_{3 / 2}\right]
\end{aligned}
$$

as well as the variation of $S_{L}$ computed after using (3.37) and (3.41)

$$
\delta S_{L}=-\int d^{3} x\left[\bar{\eta}_{-} A_{1} \not \partial \chi_{3 / 2-}+\frac{1}{L} \bar{\eta}_{+} A_{1} \chi_{3 / 2-}+\bar{\eta}_{-}\left(\frac{A_{2}}{L}-\frac{\kappa}{L}\left(A_{1}^{2}-B_{1}^{2}\right)\right) \chi_{3 / 2+}\right]
$$

We see that $\delta\left(S_{\chi}+S_{L}\right)$ can cancel $\delta\left(S_{\text {kin }}+S_{2}\right)$ in (3.53) only if $c=1$. With this choice, the sum of $(3.53)-(3.55)$ is

$$
\delta\left(S_{\mathrm{kin}}+S_{2}+S_{\chi}+S_{L}\right)=\int d^{3} x\left[-\bar{\eta}_{-}\left(\not \partial A_{1}\right) \chi_{3 / 2-}-\frac{3}{L} \bar{\eta}_{+} A_{1} \chi_{3 / 2-}-\bar{\eta}_{-} A_{1} \not \partial \chi_{3 / 2-}\right] .
$$

Finally, using $\not \partial \eta_{-}=-(3 / L) \eta_{+}$as explained below (3.23), we see that the integrand in this expression is a total derivative. Thus (3.50) follows.

\subsection{The $A B^{2}$ boundary term: a minor puzzle resolved}

While we have found a boundary term $S_{\text {bdy }}$ defined in (3.43) that ensured supersymmetry, we have not mentioned whether it is unique. In fact, if it were unique, then the following puzzle could be raised. The cubic boundary term (3.33) decomposes as

$$
S_{3}=-\frac{2 \kappa}{L} \int d^{3} x\left(A^{3}-3 A B^{2}\right)
$$


where we have indicated its form for the case $W=\kappa z^{3} /(3 L)$ as in (3.21). ${ }^{20}$ The $A^{3}$ term will be used to calculate the 3 -point correlator of three $\Delta=1$ scalar operators in the next section while the $A B^{2}$ term would generate a correlator of one scalar and two $\Delta=2$ pseudoscalars. The puzzle arises because both correlators are non-vanishing in the $\mathcal{N}=1$ models, but $\mathrm{SO}(8)$ symmetry forces ${ }^{21}\left\langle\mathcal{O}_{1}\left(\vec{x}_{1}\right) \mathcal{O}_{2}\left(\vec{x}_{2}\right) \mathcal{O}_{2}\left(\vec{x}_{3}\right)\right\rangle$ to vanish in $\mathcal{N}=8$ supergravity. This is suspicious because both the $z^{3}$ and the $z^{1} z^{2} z^{3}$ models are supposed to be consistent $\mathcal{N}=1$ truncations of $\mathcal{N}=8$.

The resolution of this issue is that the finite boundary counterterm

$$
S^{\prime}=c^{\prime} \int d^{3} x A_{1} B_{1}^{2}
$$

can be added to the $z^{3}$ model with arbitrary constant $c^{\prime}$ and maintains supersymmetry of the Legendre transform $\tilde{S}$. Further, the more general cubic polynomial

$$
\hat{S}=\int d^{3} x\left[c_{3} A_{1}^{3}+c_{2} A_{1}^{2} B_{1}+c_{1} A_{1} B_{1}^{2}+c_{0} B_{1}^{3}\right]
$$

violates supersymmetry unless $c_{0}=c_{2}=c_{3}=0$. This is quite fortunate. One can choose $c^{\prime}=c_{1}=-2 \kappa / L$ and cancel the $\left\langle\mathcal{O}_{1}\left(\vec{x}_{1}\right) \mathcal{O}_{2}\left(\vec{x}_{2}\right) \mathcal{O}_{2}\left(\vec{x}_{3}\right)\right\rangle$ correlator which must vanish in a consistent truncation of $\mathcal{N}=8$, while the coefficient of $\left\langle\mathcal{O}_{1}\left(\vec{x}_{1}\right) \mathcal{O}_{1}\left(\vec{x}_{2}\right) \mathcal{O}_{1}\left(\vec{x}_{3}\right)\right\rangle$ retains the value which matches the non-perturbative physics of the boundary $\mathcal{N}=8 \mathrm{SCFT}$.

It is easy to establish the facts mentioned above. In particular:

1. The addition of the boundary term $\hat{S}$ requires that we recompute the extremal point of $\tilde{S}$. We find that $\mathfrak{A}$ shifts as $\mathfrak{A} \rightarrow \mathfrak{A}+\hat{\mathfrak{A}}$ with

$$
\hat{\mathfrak{A}}=-\left(3 c_{3} A_{1}^{2}+2 c_{2} A_{1} B_{1}+c_{1} B_{1}^{2}\right) .
$$

The boundary term $S_{L}$ shifts as $S_{L} \rightarrow S_{L}+\hat{S}_{L}$ with $\hat{S}_{L}=\int d^{3} x \hat{\mathfrak{A}} A_{1}$.

2. These changes are compatible with supersymmetry if

$$
\begin{aligned}
\delta\left(\hat{S}+\hat{S}_{L}\right) & =\int d^{3} x\left[\delta\left(c_{3} A_{1}^{3}+c_{2} A_{1}^{2} B_{1}+c_{1} A_{1} B_{1}^{2}+c_{0} B_{1}^{3}\right)+\delta \hat{\mathfrak{A}} A_{1}+\hat{\mathfrak{A}} \delta A_{1}\right] \\
& =\int d^{3} x\left[-2 A_{1}\left(c_{2} B_{1}+3 c_{3} A_{1}\right) \delta A_{1}+\left(3 B_{1}^{2} c_{0}-A_{1}^{2} c_{2}\right) \delta B_{1}\right]=0 .
\end{aligned}
$$

where we used (3.60), and where $\delta A_{1}$ and $\delta B_{1}$ are understood to be computed from (3.26). It is then straightforward to determine the integrand of (3.61) and observe that it vanishes if and only if $c_{0}=c_{2}=c_{3}=0$, while $c_{1}$ is arbitrary.

\footnotetext{
${ }^{20}$ When $W=\kappa z^{1} z^{2} z^{3} / L$ the r.h.s. of this expression contains the combination $A^{1} A^{2} A^{3}-A^{1} B^{2} B^{3}-$ $A^{2} B^{3} B^{1}-A^{3} B^{1} B^{2}$.

${ }^{21}$ The subscripts here indicate scale dimension.
} 


\subsection{Boundary SUSY for $\mathcal{N}=1$ truncations of supergravity.}

In this section we discuss, qualitatively, the steps that are needed to show that the boundary terms obtained above in the global limit are not changed by reanalysis at the level of $\mathcal{N}=1$ supergravity. In supergravity we must use $\epsilon(r, \vec{x})$ parameters with arbitrary dependence on the coordinates of the bulk theory. The terms in the general $\mathcal{N}=1$ Lagrangian (as presented in (18.6) of [60]) that must be considered are the chiral multiplet terms that have obvious limits to the global Lagrangian in [36]. These include, respectively, the $m_{3 / 2}$ and $m_{\alpha \beta}$ terms in (18.15) and (18.16) of [60]. We must also include the Noether current term (written for a single multiplet)

$$
\mathcal{L}_{\text {Noether }}=\frac{1}{\sqrt{2}} \bar{\psi}_{\mu}\left[\left(\not \nabla \bar{z} \gamma^{\mu}+\gamma^{\mu} \nabla W_{\mathrm{SG}}\right) P_{L} \chi+\text { c.c. }\right],
$$

and use its gravitino variation $\delta \psi_{\mu}=\sqrt{2}\left(\nabla_{\mu} \epsilon+\frac{1}{2 L} \gamma_{\mu}\right) \epsilon$. We can drop terms in the supergravity Lagrangian, such as the quartic fermion terms, and in transformation rules, whose contribution to possible boundary terms vanishes when the AdS/CFT asymptotic conditions of (3.25) are used. ${ }^{22}$

With the action and transformation rules limited in this manner, one finds that the $\nabla_{\mu} \epsilon$ terms from $\delta \mathcal{L}_{\text {Noether }}$ combine with others elsewhere in $\delta S$ to produce the same set of boundary terms found in (3.19) and (3.20), but with general spinor parameters $\epsilon(r, \vec{x})$. The assumption that they approach Killing spinors as $r \rightarrow \infty$ is then used to study the boundary terms in more detail.

\section{2 - and 3-point correlators from $\mathcal{N}=1$ supergravity}

In this section we present a holographic calculation of 2- and 3-point functions of a $\Delta=1$ CFT operator $\mathcal{O}_{1}$ in the example (3.14) from $\mathcal{N}=1$ supergravity with the cubic superpotential (3.21). The computation in this toy model will be generalized to $\mathcal{N}=8$ supergravity in section 8 .

\subsection{2- and 3-point correlators}

As we explained in the previous section, the operator $\mathcal{O}_{1}$ is dual to the bulk scalar $A=\operatorname{Re} z$. The pseudoscalar field $B$ and fermion $\chi$ play no role in the calculation of correlators of $\mathcal{O}_{1}$, so we set them to zero. The part of the action (3.14) involving $A$ and the boundary term (3.32) that we need is

$$
S=\frac{1}{2} \int d^{4} x \sqrt{g}\left[\partial_{\mu} A \partial^{\mu} A-\frac{2}{L^{2}} A^{2}\right]+\frac{1}{2 L} \int d^{3} x e^{3 r_{0} / L}\left[A^{2}+\frac{2 \kappa}{3} A^{3}\right],
$$

where we Wick rotated to Euclidean signature and multiplied (3.14) by an overall factor of $1 / 2$ for a more conventional normalization. We set $\kappa=L=1$ in this section.

The field $A$ obeys the equation of motion

$$
(\square+2) A=0 .
$$

\footnotetext{
${ }^{22}$ Boundary conditions on the gravitino are not needed for our purposes. They are discussed in [55-59].
} 
As in (3.25), the solution of this equation of motion can be expanded at large $r$ as

$$
A(r, \vec{x})=e^{-r} A_{1}(\vec{x})+e^{-2 r} A_{2}(\vec{x})+\cdots .
$$

In fact, the equation of motion (4.2) implies that the entire bulk field can be reconstructed in terms of $A_{1}(\vec{x})$ with the help of the bulk-to-boundary propagator

$$
A(r, \vec{x})=\int d^{3} y K_{2}(r, \vec{x} ; \vec{y}) A_{1}(\vec{y}), \quad K_{2}(r, \vec{x} ; \vec{y}) \equiv \frac{1}{\pi^{2}} \frac{e^{-2 r}}{\left(e^{-2 r}+|\vec{x}-\vec{y}|^{2}\right)^{2}}
$$

Plugging this expression into (4.1) one obtains the on-shell action written as a functional of the boundary coefficient $A_{1}(\vec{x}):{ }^{23}$

$$
S_{\text {on-shell }}\left[A_{1}\right]=-\frac{1}{2} \int d^{3} x d^{3} y \frac{A_{1}(\vec{x}) A_{1}(\vec{y})}{\pi^{2}|\vec{x}-\vec{y}|^{4}}+\frac{1}{3} \int d^{3} x A_{1}(\vec{x})^{3}+O\left(A_{1}^{4}\right) .
$$

This expression would be the goal of our computation if $A$ were dual to a dimension 2 field theory operator $\mathcal{O}_{2}$. In that case $A_{1}(\vec{x})$ would be interpreted as the source of $\mathcal{O}_{2}$, and, by the AdS/CFT dictionary, $-S_{\text {on-shell }}\left[A_{1}\right]$ becomes the generating functional of its connected correlators, certainly not what we want.

Indeed, in our case of interest, the field $A$ is dual to a dimension 1 operator $\mathcal{O}_{1}$, but $A_{1}(\vec{x})$ is not the field theory source for $\mathcal{O}_{1}$. Instead, the field theory source, denoted $\mathfrak{A}(\vec{x})$, is the canonically conjugate variable to $A_{1}[25]$ and the generating functional is the Legendre transform $\tilde{S}_{\text {on-shell }}[\mathfrak{A}]$ defined as in (3.39) by

$$
\tilde{S}_{\text {on-shell }}[\mathfrak{A}]=S_{\text {on-shell }}\left[A_{1}\right]+\int d^{3} x \mathfrak{A}(\vec{x}) A_{1}(\vec{x}) .
$$

These ideas were introduced in section 3.6, where our main purpose was to demonstrate that the generating functional $\tilde{S}_{\text {on-shell }}\left[B_{1}, \chi_{3 / 2-}, \mathfrak{A}\right]$ is a supersymmetric functional of its sources. In this section our purpose is more pragmatic; we wish to express $\tilde{S}_{\text {on-shell }}[\mathfrak{A}]$ in a form in which functional derivatives with respect to $\mathfrak{A}(\vec{x})$ can be applied to produce correlators of $\mathcal{O}_{1}$.

Toward that end, we proceed to extremize the r.h.s. of (4.6) with respect to $A_{1}(\vec{x})$ after inserting the toy model expression (4.5). Extremization yields the result

$$
\mathfrak{A}(\vec{x})=-\frac{\delta S_{\text {on-shell }}\left[A_{1}\right]}{\delta A_{1}(\vec{x})}=\frac{1}{\pi^{2}} \int d^{3} y \frac{A_{1}(\vec{y})}{|\vec{x}-\vec{y}|^{4}}-A_{1}(\vec{x})^{2}+O\left(A_{1}^{3}\right) .
$$

This expression can be inverted by taking its convolution with $1 /\left(2 \pi^{2}|\vec{z}-\vec{x}|^{2}\right)$ and using the relation

$$
\int d^{3} x \frac{1}{2 \pi^{2}|\vec{z}-\vec{x}|^{2}} \frac{1}{\pi^{2}|\vec{x}-\vec{y}|^{4}}=-\delta^{(3)}(\vec{z}-\vec{y})
$$

\footnotetext{
${ }^{23}$ Here and in the following formulas, the integration kernel $\frac{1}{|\vec{x}-\vec{y}|^{4}}$ is understood to be regularized by replacing it with $\frac{1}{\left(\epsilon^{2}+|\vec{x}-\vec{y}|^{2}\right)^{2}}$, where $\epsilon=e^{-r_{0}}$ is a holographic UV cutoff, and discarding the power divergences in $\epsilon$. Discarding such power divergences can be unambiguously done in a CFT.
} 
which can be derived, for instance, by passing to Fourier space. ${ }^{24}$ The expression for $A_{1}(\vec{x})$ in terms of $\mathfrak{A}(\vec{x})$ is finally

$$
A_{1}(\vec{x})=-\int d^{3} y \frac{\mathfrak{A}(\vec{y})}{2 \pi^{2}|\vec{x}-\vec{y}|^{2}}-\frac{1}{\left(2 \pi^{2}\right)^{3}} \int d^{3} y d^{3} z \mathfrak{A}(\vec{y}) \mathfrak{A}(\vec{z}) I(\vec{x}, \vec{y}, \vec{z})+O\left(\mathfrak{A}^{3}\right),
$$

where

$$
I(\vec{x}, \vec{y}, \vec{z})=\int d^{3} w \frac{1}{|\vec{x}-\vec{w}|^{2}|\vec{y}-\vec{w}|^{2}|\vec{z}-\vec{w}|^{2}}=\frac{\pi^{3}}{|\vec{x}-\vec{y}||\vec{y}-\vec{z}||\vec{x}-\vec{z}|} .
$$

Plugging this into (4.6) and using (4.10) again gives

$$
\tilde{S}_{\text {on-shell }}[\mathfrak{A}]=-\frac{1}{4 \pi^{2}} \int d^{3} x d^{3} y \frac{\mathfrak{A}(\vec{x}) \mathfrak{A}(\vec{y})}{|\vec{x}-\vec{y}|^{2}}-\frac{1}{24 \pi^{3}} \int d^{3} x d^{3} y d^{3} z \frac{\mathfrak{A}(\vec{x}) \mathfrak{A}(\vec{y}) \mathfrak{A}(\vec{z})}{|\vec{x}-\vec{y}||\vec{y}-\vec{z}||\vec{x}-\vec{z}|}+O\left(\mathfrak{A}^{4}\right) .
$$

The first term in this expression agrees with the result of [25] in a free bulk theory. The expression (4.11) thus generalizes this result to include a cubic boundary interaction.

Since $-\tilde{S}_{\text {on-shell }}[\mathfrak{A}]$ is interpreted as the generating function of connected correlators for the operator $\mathcal{O}_{1}$, we obtain the 2- and 3- point functions

$$
\begin{aligned}
\left\langle\mathcal{O}_{1}\left(\vec{x}_{1}\right) \mathcal{O}_{1}\left(\vec{x}_{2}\right)\right\rangle & =\frac{1}{2 \pi^{2}\left|\vec{x}_{1}-\vec{x}_{2}\right|^{2}}, \\
\left\langle\mathcal{O}_{1}\left(\vec{x}_{1}\right) \mathcal{O}_{1}\left(\vec{x}_{2}\right) \mathcal{O}_{1}\left(\vec{x}_{3}\right)\right\rangle & =\frac{1}{4 \pi^{3}} \frac{1}{\left|\vec{x}_{1}-\vec{x}_{2}\right|\left|\vec{x}_{2}-\vec{x}_{3}\right|\left|\vec{x}_{1}-\vec{x}_{3}\right|} .
\end{aligned}
$$

In section 8 , a similar computation is used to obtain the 2- and 3-point functions of the dimension 1 operators of an $\mathcal{N}=8$ SCFT transforming in the $\mathbf{3 5}_{v}$ representation of $\mathrm{SO}(8)$ R-symmetry.

\subsection{On the nonlinear boundary condition for $A(r, \vec{x})$}

The toy model provides the opportunity to explore the Legendre transform further and hopefully gain further insight into its workings. Toward that end we express the bulk field $A(r, \vec{x})$ in terms of boundary data for a source $\mathfrak{A}(\vec{x})$ of compact support. We then study its boundary limit in a region where the source vanishes and show explicitly that the boundary condition

$$
\mathfrak{A}(\vec{x})=A_{2}(\vec{x})-A_{1}(\vec{x})^{2}=0, \quad \vec{x} \in(\operatorname{supp}(\mathfrak{A}))^{c}
$$

is satisfied.

The bulk field $A(r, \vec{x})$ can be expressed in terms of the boundary data $\mathfrak{A}(\vec{x})$ by combining (4.4) and (4.9). Performing the required integrals, one can write the resulting expression as

$$
A(r, \vec{x})=\int d^{3} y K_{1}(r, \vec{x} ; \vec{y})\left(\mathfrak{A}(\vec{y})+\frac{1}{\left(2 \pi^{2}\right)^{2}} \int d^{3} z d^{3} w \frac{\mathfrak{A}(\vec{z}) \mathfrak{A}(\vec{w})}{|\vec{y}-\vec{z}|^{2}|\vec{y}-\vec{w}|^{2}}\right)+O\left(\mathfrak{A}^{3}\right),
$$

${ }^{24} \mathrm{~A}$ more careful regulated analysis gives

$$
\int d^{3} x \frac{1}{2 \pi^{2}|\vec{z}-\vec{x}|^{2}} \frac{1}{\pi^{2}\left(\epsilon^{2}+|\vec{x}-\vec{y}|^{2}\right)^{2}}=\frac{1}{\epsilon} \frac{1}{2 \pi^{2}|\vec{z}-\vec{y}|^{2}}-\delta^{(3)}(\vec{z}-\vec{y}) .
$$

To derive (4.8) one must discard the linear UV divergence. The regulated expression above is simply a combination of (4.15) and (4.17) below multiplied by $\epsilon^{2}=e^{-2 r_{0}}$. 
where

$$
K_{1}(r, \vec{x} ; \vec{y}) \equiv \int d^{3} z K_{2}(r, \vec{x} ; \vec{z}) \frac{-1}{2 \pi^{2}|\vec{z}-\vec{y}|^{2}}=-\frac{1}{2 \pi^{2}} \frac{e^{-r}}{e^{-2 r}+|\vec{x}-\vec{y}|^{2}}
$$

To check (4.13), we should expand $A(r, \vec{x})$ at large $r$ and assume that $\vec{x}$ lies outside the support of $\mathfrak{A}$. Since $r$ only appears in $K_{1}$, we can expand $K_{1}$ at large $r$ first. To leading order in $e^{-r}$, we have that $K_{1}(r, \vec{x} ; \vec{y})$ approaches $-\frac{e^{-r}}{2 \pi^{2}|\vec{x}-\vec{y}|^{2}}$. The first subleading correction can be computed as

$$
e^{2 r}\left(K_{1}(r, \vec{x} ; \vec{y})+\frac{e^{-r}}{2 \pi^{2}|\vec{x}-\vec{y}|^{2}}\right)=\frac{1}{2 \pi^{2}} \frac{e^{-r}}{|\vec{x}-\vec{y}|^{2}\left(e^{-2 r}+|\vec{x}-\vec{y}|^{2}\right)} \rightarrow \delta^{(3)}(\vec{x}-\vec{y}) .
$$

So

$$
K_{1}(r, \vec{x} ; \vec{y}) \rightarrow-\frac{e^{-r}}{2 \pi^{2}|\vec{x}-\vec{y}|^{2}}+e^{-2 r} \delta^{(3)}(\vec{x}-\vec{y}) .
$$

Using this large $r$ expansion in (4.14) and comparing with (4.3), we identify

$$
\begin{aligned}
& A_{1}(\vec{x})=-\frac{1}{2 \pi^{2}} \int d^{3} y \frac{\mathfrak{A}(\vec{y})}{|\vec{x}-\vec{y}|^{2}}+O\left(\mathfrak{A}^{2}\right), \\
& A_{2}(\vec{x})=\frac{1}{\left(2 \pi^{2}\right)^{2}} \int d^{3} z d^{3} w \frac{\mathfrak{A}(\vec{z}) \mathfrak{A}(\vec{w})}{|\vec{x}-\vec{z}|^{2}|\vec{x}-\vec{w}|^{2}}+O\left(\mathfrak{A}^{3}\right) .
\end{aligned}
$$

By examining (4.18) it is easy to see that, indeed, the non-linear boundary condition (4.13) is obeyed.

\section{The $\mathcal{N}=8$ supergravity}

We begin with a brief summary of the $\mathcal{N}=8$ gauged supergravity in four dimensions $[1,65]$ with the $\mathrm{SO}(8)$ gauge fields set to zero. In the bosonic sector one is then left with the metric, $g_{\mu \nu}$, and the scalar/pseudoscalar fields parametrizing the non-compact coset space $\mathrm{E}_{7(7)} / \mathrm{SU}(8)$. In the symmetric gauge $[1,66]$, the scalar 56 -bein, $\mathcal{V}$, is explicitly given by

$$
\mathcal{V} \equiv\left(\begin{array}{cc}
u_{i j}^{I J} & v_{i j I J} \\
v^{k l I J} & u^{k l} K L
\end{array}\right)=\exp \left(\begin{array}{cc}
0 & -\frac{1}{4} \sqrt{2} \phi_{i j k l} \\
-\frac{1}{4} \sqrt{2} \phi^{i j k l} & 0
\end{array}\right) \in \mathrm{E}_{7(7)}
$$

where

$$
\phi_{i j k l}=\frac{1}{24} \epsilon_{i j k l m n p q} \phi^{m n p q}, \quad \phi^{i j k l}=\left(\phi_{i j k l}\right)^{*},
$$

are complex self-dual fields, whose real and imaginary parts, $\phi_{i j k l}=\alpha^{i j k l}+i \beta^{i j k l}$, are the $35_{v}$ scalars, $\alpha^{i j k l}$, and $35_{c}$ pseudoscalars, $\beta^{i j k l}$, respectively, where the labels $s$ and $c$ indicate the assignment of $\mathrm{SO}(8)$ representations. ${ }^{25}$ In the fermionic sector, there are $8_{s}$ left/right-handed gravitini, $\psi_{\mu}{ }^{i} / \psi_{\mu i}$, and $56_{s}$ left/right-handed gauginos $\chi^{i j k} / \chi_{i j k}$. As for complex scalars, see (5.2), complex conjugation of the fermions amounts simply to

\footnotetext{
${ }^{25}$ See, e.g., table 7 in [67].
} 
raising/lowering of the $\mathrm{SU}(8)$ indices, for example $\left(\chi_{i j k}\right)^{*}=\chi^{i j k}$, with the corresponding change of chirality.

The scalar fields enter the action and the supersymmetry transformations through the composite $\mathrm{SU}(8)$ connection, $\mathcal{B}_{\mu}{ }^{i}{ }^{j}$, and the self-dual tensor, $\mathcal{A}_{\mu}{ }^{i j k l}$, defined by [66]:

$$
\begin{aligned}
\mathcal{B}_{\mu j}{ }^{i} & =\frac{2}{3}\left(u^{i k}{ }_{I J} \partial_{\mu} u_{j k}{ }^{I J}-v^{i k I J} \partial_{\mu} v_{j k I J}\right), \\
\mathcal{A}_{\mu}{ }^{i j k l} & =-2 \sqrt{2}\left(u^{i j}{ }_{I J} \partial_{\mu} v^{k l I J}-v^{i j I J} \partial_{\mu} u^{k l}{ }_{I J}\right),
\end{aligned}
$$

and the two $A$-tensors [1]:

$$
A_{1}^{i j}=\frac{4}{21} T_{k}^{i k j}, \quad A_{2 i}{ }^{j k l}=-\frac{4}{3} T_{i}^{[j k l]},
$$

defined in terms of the $T$-tensor,

$$
T_{i}^{j k l} \equiv\left(u_{I J}^{k l}+v^{k l I J}\right)\left(u_{i m}^{J K} u_{K I}^{j m}-v_{i m J K} v^{j m K I}\right) .
$$

Note that $A_{1}{ }^{i j}=A_{1}{ }^{j i}$, while $A_{2 i}{ }^{j k l}=A_{2 i}{ }^{[j k l]}$.

The bosonic action of the $\mathcal{N}=8$ supergravity in the gravity plus scalar sector is $[1]^{26}$

$$
S_{\mathrm{B}}=\int d^{4} x \sqrt{-g}\left[\frac{1}{2} R-\frac{1}{96} \mathcal{A}_{\mu}{ }^{i j k l} \mathcal{A}^{\mu}{ }_{i j k l}-g^{2} \mathcal{P}\right]
$$

where

$$
\mathcal{P}=-\frac{3}{4}\left|A_{1}{ }^{i j}\right|^{2}+\frac{1}{24}\left|A_{2} l^{i j k}\right|^{2},
$$

is the scalar potential. The maximally supersymmetric solution is given by the $\mathrm{AdS}_{4}$ metric (3.10) of radius

$$
L=\frac{1}{\sqrt{2} g},
$$

and vanishing scalar fields, $\phi_{i j k l}=0$.

For a general solution, the asymptotic expansion of the scalar fields is similar to that in (3.25), namely

$$
\begin{aligned}
\phi^{i j k l}(r, \vec{x}) & =e^{-r / L} \phi_{(1)}{ }^{i j k l}(\vec{x})+e^{-2 r / L} \phi_{(2)}{ }^{i j k l}(\vec{x})+\ldots, \\
\phi_{(n)}{ }^{i j k l}(\vec{x}) & =\alpha_{(n)}{ }^{i j k l}(\vec{x})-i \beta_{(n)}{ }^{i j k l}(\vec{x}) .
\end{aligned}
$$

Using the symmetric gauge (5.1) and the definitions (5.3)-(5.5), one can verify by a somewhat tedious calculation the following expansions of the composite fields [66]:

$$
\begin{aligned}
\mathcal{B}_{\mu}{ }^{i}{ }_{j} & =-\frac{1}{24}\left(\phi^{i p q r} \partial_{\mu} \phi_{j p q r}-\phi_{j p q r} \partial_{\mu} \phi^{i p q r}\right)+O\left(\phi^{4}\right), \\
\mathcal{A}_{\mu}{ }^{i j k l} & =\partial_{\mu} \phi^{i j k l}+\frac{1}{24} \phi_{p q r s} \phi^{p q[i j} \partial_{\mu} \phi^{k l] r s}-\frac{1}{24} \phi^{p q[i j} \phi^{k l] r s} \partial_{\mu} \phi_{p q r s}+O\left(\phi^{5}\right),
\end{aligned}
$$

\footnotetext{
${ }^{26}$ In this section we set $\kappa^{2}=1 / 8 \pi G_{4}=1$.
} 
and of the $A$-tensors [65], ${ }^{27}$

$$
\begin{aligned}
A_{1}{ }^{i j} & =\left(1+\frac{1}{192}|\phi|^{2}\right) \delta^{i j}+\frac{\sqrt{2}}{96} \phi^{i k m n} \phi_{m n p q} \phi^{p q k j}+O\left(\phi^{4}\right), \\
A_{2 l} l^{i j k} & =-\frac{\sqrt{2}}{2}\left(1+\frac{1}{144}|\phi|^{2}\right) \phi^{i j k l}-\frac{3}{8} \phi_{m n l[i} \phi^{j k] m n}+\frac{\sqrt{2}}{16} \phi_{l p q r} \phi^{p q s[i} \phi^{j k] r s}+O\left(\phi^{4}\right),
\end{aligned}
$$

where $|\phi|^{2}=\phi_{i j k l} \phi^{i j k l}$. In particular, it follows from (5.12) and (5.13) that

$$
\begin{aligned}
\left|A_{1}{ }^{i j}\right|^{2} & =8+\frac{1}{12}\left|\phi^{2}\right|-\frac{\sqrt{2}}{96}\left(\phi^{i j k l} \phi_{k l m n} \phi^{m n i j}+\text { c.c. }\right)+O\left(\phi^{4}\right), \\
\left|A_{2} l^{i j k}\right|^{2} & =\frac{1}{2}|\phi|^{2}-\frac{3 \sqrt{2}}{16}\left(\phi^{i j k l} \phi_{k l m n} \phi^{m n i j}+\text { c.c. }\right)+O\left(\phi^{4}\right) .
\end{aligned}
$$

Hence the scalar potential (5.8),

$$
\mathcal{P}=-6-\frac{1}{24}|\phi|^{2}+O\left(\phi^{4}\right),
$$

has no cubic terms in its expansion! This is the source of the puzzle we resolve in this paper.

In the following we will also need the action for the spin- $1 / 2$ fields:

$$
\begin{aligned}
S_{\chi \text {-bulk }}=\int d^{4} x \sqrt{-g}[ & -\frac{1}{12}\left(\bar{\chi}^{i j k} \gamma^{\mu} D_{\mu} \chi_{i j k}+\bar{\chi}_{i j k} \gamma^{\mu} D_{\mu} \chi^{i j k}\right) \\
& \left.+\frac{\sqrt{2}}{144} g\left(\epsilon^{i j k p q r l m} A_{2}^{n}{ }_{p q r} \bar{\chi}_{i j k} \chi_{l m n}+\text { c.c. }\right)\right],
\end{aligned}
$$

and their Noether coupling to the gravitini:

$$
S_{\text {Noether }}=\int d^{4} x \sqrt{-g}\left[-\frac{1}{12} \mathcal{A}_{\mu}{ }^{i j k l} \bar{\chi}_{i j k} \gamma^{\nu} \gamma^{\mu} \psi_{\nu l}+\frac{g}{6} A_{2}^{i}{ }_{j k l} \bar{\psi}_{\mu i} \gamma^{\mu} \chi^{j k l}+\text { c.c. }\right] \text {. }
$$

The supersymmetry variation of the scalar fields is [1]

$$
\left(\delta \mathcal{V} \mathcal{V}^{-1}\right)_{i j k l} \equiv-2 \sqrt{2} \Sigma_{i j k l}
$$

where

$$
\Sigma_{i j k l}=\bar{\epsilon}_{\left[i \chi_{j k l]}\right.}+\frac{1}{24} \eta_{i j k l m n p q} \bar{\epsilon}^{m} \chi^{n p q},
$$

is self-dual. The expansion of (5.18) yields the result similar to $(5.11)$ [1, 66], namely,

$$
\delta \phi_{i j k l}=8 \Sigma_{i j k l}\left(1+O\left(\phi^{2}\right)\right) .
$$

Finally, the supersymmetry variations of the left-handed gravitinos and gauginos in the $\mathcal{N}=8$ theory are given by $[1]$

$$
\begin{aligned}
\delta \psi_{\mu}{ }^{i} & =2 D_{\mu} \epsilon^{i}+\sqrt{2} g A_{1}{ }^{i j} \gamma_{\mu} \epsilon_{j}, \\
\delta \chi^{i j k} & =-\mathcal{A}_{\mu}{ }^{i j k l} \gamma^{\mu} \epsilon_{l}-2 g A_{2}{ }^{i j k} \epsilon^{l},
\end{aligned}
$$

with the corresponding complex conjugate variations of the right-handed fields, $\psi_{\mu i}$ and $\chi_{i j k}$.

\footnotetext{
${ }^{27}$ We correct the sign in the first bracket on the right hand side in (5.13).
} 
To conclude this summary we note that the bosonic action (5.7) expanded about its maximally supersymmetric solution is

$$
S_{\mathrm{B}}=\int d^{4} x\left[\frac{1}{2} R-\frac{1}{96} \partial_{\mu} \phi_{i j k l} \partial^{\mu} \phi^{i j k l}+g^{2}\left(6+\frac{1}{24}|\phi|^{2}\right)+\ldots\right],
$$

and has the same structure as the corresponding $\mathcal{N}=1$ action in (3.29). This suggests that we should find the boundary counterterms with the same structure as those found in section 3. To determine them we first consider the supersymmetry transformations of the fermions and the corresponding Bogomolny factorization as in section 3.1 and then confirm the result by a direct supergravity calculation.

\section{Bogomolny argument in $\mathcal{N}=8$ supergravity}

\subsection{Motivation}

It is useful to recall the form of the original BPS arguments [68, 69]. These are computations in field theories in flat backgrounds and, at least for monopoles, involve completing the square in the Hamiltonian. This completion of the square requires boundary terms that bound the energy from below. The bound is saturated precisely when the perfect square in the bulk action vanishes and this condition leads to the BPS equations. Apart from time-independence, there were no special assumptions about how the fields depended on coordinates and the original treatment involved flat space and did not incorporate gravitational back-reaction.

In this section, we will make a "BPS-inspired" argument by making a similar completion of squares, but there will be several important differences with the standard BPS story. First, our metric will not be flat but will be that of the "kink Ansatz," (3.1), the most general metric that preserves Poincaré invariance in the boundary directions. However, unlike (3.1), we will consider completely general scalar fields. We use this metric Ansatz because we wish to consider fields in AdS and in asymptotically-AdS backgrounds.

Exactly as in the BPS story, we will complete the square in the bulk action and collect the essential boundary terms that are needed to achieve this. Since we are allowing a non-trivial scale factor, $\mathcal{A}(r)$, in our metric Ansatz, one should anticipate that the energy will not be bounded below. Indeed, one finds that the bulk action produces a signed sum of squares. Thus, unlike the BPS story, we cannot obtain a lower bound on the energy. What is important here is that we show that the action with the completed squares in the bulk has much better fall-off behavior at infinity in an asymptotically-AdS background. The result is that the boundary terms obtained from the "BPS-inspired" completion of squares are precisely the boundary terms that one needs to regulate the action in an asymptotically-AdS background.

\subsection{The BPS equations in the "kink Ansatz"}

As in section 3, we start by assuming that the metric has the Poincaré-invariant form (3.1) and that the scalar fields depend only on the radial coordinate, $r$. Setting the spacetime 
components $(\mu=0,1,2)$ of the gravitino variations (5.21) and the gaugino variations (5.22) to zero, we obtain the following equations:

$$
\begin{aligned}
\mathcal{A}^{\prime} \gamma^{3} \epsilon^{i}+\sqrt{2} g A_{1}{ }^{i j} \epsilon_{j} & =0, \\
-\mathcal{A}_{r}{ }^{i j k l} \gamma^{3} \epsilon_{l}-2 g A_{2} l^{i j k} \epsilon^{l} & =0,
\end{aligned}
$$

which, together with the complex conjugate equations, constitute a linear system for the Killing spinors, $\epsilon_{i}$ and $\epsilon^{i}$.

Motivated by the known solutions to (6.1) from RG-flows in various truncations of the $\mathcal{N}=8$ theory (see for example $[62,70-75]$ ), let us set

$$
\gamma^{3} \epsilon^{i}=X^{i j} \epsilon_{j}, \quad \gamma^{3} \epsilon_{i}=X_{i j} \epsilon^{j}, \quad X^{i j}=\left(X_{i j}\right)^{*}
$$

where $X_{i j}$ is a symmetric matrix, which by consistency with $\left(\gamma^{3}\right)^{2}=I$ must also be unitary. Then, substituting (6.2) in (6.1), we find the following equations:

$$
\left(\mathcal{A}^{\prime} X^{i j}+\sqrt{2} g A_{1}^{i j}\right) \epsilon_{j}=0, \quad\left(\mathcal{A}_{r}^{i j k l}+2 g X^{l m} A_{2 m}^{i j k}\right) \epsilon_{l}=0,
$$

where the matrices acting on the Killing spinors, $\epsilon_{i}$, are the BPS operators we are looking for.

We refer the reader to appendices $\mathrm{C}$ and $\mathrm{E}$ for further discussion of truncations and flows. Here let us note that for known RG flows the components of the BPS operators that act on the nonvanishing $\epsilon_{i}$ 's reduce to the usual BPS equations for the metric function and the scalar fields, respectively. In particular, in the truncation discussed in [14] (see also appendix E) they yield the BPS equations (3.3).

In the following we will show that the BPS operators defined in (6.3) provide natural factors for the $\mathcal{N}=8$ analogue of the Bogomolny argument in section 3 . At the same time one should keep in mind that the discussion below is completely general and independent of any solution of (6.3). In particular, the factorization in section 6.3 holds for scalar fields with arbitrary space-time dependence. All that we use is that the metric has the form (3.1), the general form of the BPS operators and identities satisfied by the $A$-tensors in $\mathcal{N}=8, d=4$ supergravity.

\subsection{Completing the square}

We now generalize the result: the metric will still be required to be of the form (3.1), but the scalar fields will be allowed to have arbitrary dependence on all coordinates. With these choices, the bosonic action (5.7) reduces to the following effective action for the scalars and gravitational field: ${ }^{28}$

$$
S_{\mathrm{B}}=\int d^{4} x e^{3 A}\left[3\left(\mathcal{A}^{\prime}\right)^{2}+\frac{3}{4} g^{2}\left|A_{1}{ }^{i j}\right|^{2}-\frac{1}{96} \mathcal{A}_{\mu}{ }^{i j k l} \mathcal{A}^{\mu}{ }_{i j k l}-\frac{1}{24} g^{2}\left|A_{2 i}{ }^{j k l}\right|^{2}\right] .
$$

\footnotetext{
${ }^{28}$ The reduction of (5.7) to (6.4) introduces boundary terms that arise from the integration by parts of the second order derivatives of the metric inside the Ricci scalar. Those terms are then cancelled by the usual Gibbons-Hawking boundary counterterm.
} 
At first sight, it may seem inconsistent to employ such an action because scalars depending on the boundary directions will have an energy momentum tensor that sources metric components that violate the metric Anstaz in (3.1). There are two, essentially equivalent, ways to think about this. First, we want to work about a gravitational background that preserves Poincaré invariance in the boundary directions and, as in section 3, we want to "consistently suppress" all gravitational back-reaction that breaks the Poincaré invariance. This can be reduced to a prescription in terms of powers of the gravitational coupling, $\kappa$, but we can simply take the view that we use (6.4) and drop all the Einstein equations involving components of the energy-momentum tensor that break Poincaré invariance.

The second, and more practical perspective, is that our goal now is to examine the behavior of the action in asymptotically-AdS space and consider the asymptotic behavior of the bulk action as it approaches the boundary. To that end, we note that (6.4) contains precisely the degrees of freedom that remain non-trivial as the metric asymptotes that of AdS at infinity. We discuss this more in section 6.5.

The supersymmetry means that this action can be written in terms of squares of the BPS operators introduced above. Indeed, the first two terms in (6.4) may be written as

$$
\begin{aligned}
e^{3 A}\left[3\left(\mathcal{A}^{\prime}\right)^{2}+\frac{3}{4} g^{2}\left|A_{1}{ }^{i j}\right|^{2}\right]= & \frac{3}{8} e^{3 A}\left|\mathcal{A}^{\prime} X_{i j} \mp \sqrt{2} g A_{1 i j}\right|^{2} \\
& \pm \frac{3}{4 \sqrt{2}} g \mathcal{A}^{\prime} e^{3 A}\left[X_{i j} A_{1}^{i j}+X^{i j} A_{1 i j}\right]
\end{aligned}
$$

where $X_{i j}$ is any unitary matrix. Similarly, the $A_{2}$-term and the radial component of the scalar kinetic term in (6.4) can be written as:

$$
\begin{aligned}
e^{3 A}\left[-\frac{1}{96} \mathcal{A}_{r}{ }^{i j k l} \mathcal{A}_{r i j k l}-\frac{g^{2}}{24}\left|A_{2 i}{ }^{j k l}\right|^{2}\right]= & -\frac{1}{96} e^{3 A}\left|\mathcal{A}_{r}{ }^{i j k l} \pm 2 g X^{i m} A_{2 m}{ }^{j k l}\right|^{2} \\
& \pm \frac{g}{48} e^{3 A}\left[\mathcal{A}_{r i j k l} X^{i m} A_{2 m}{ }^{j k l}+\mathcal{A}_{r}{ }^{i j k l} X_{i m} A_{2}{ }^{m}{ }_{j k l}\right] .
\end{aligned}
$$

The role of the dynamical matrix $X_{i j}$ here is to preserve the $\mathrm{SU}(8)$ covariance of the factors inside the squares. In principle, we could choose $X_{i j}$ in any convenient manner and one could even choose these matrices to be different in (6.5) and (6.6).

There is, however, a very natural and canonical choice that is motivated by flows and superpotentials of truncated theories. We will also see that this choice also leads to a very simple boundary action. Autonne-Takagi factorization [76] allows one to write the symmetric, complex matrix, $A_{1}{ }^{i j}$, as

$$
A_{1}^{i j}=\left(S D S^{T}\right)^{i j},
$$

where $S^{i}{ }_{j}$ is a unitary matrix and $D^{i j}$ is real and diagonal with non-negative eigenvalues. Indeed, multiplying this by its complex conjugate gives

$$
A_{1}{ }^{i k} A_{1 k j}=\left(S D^{2} S^{\dagger}\right)^{i}{ }_{j} .
$$

and so the eigenvalues of $D$ are the square-roots of the real eigenvalues of the hermitian matrix $A_{1}{ }^{i k} A_{1 k j}$. Choose

$$
X_{i j}=\left(S^{*} S^{\dagger}\right)_{j i}, \quad X^{i j}=\left(X_{i j}\right)^{*} .
$$


Note that $X_{i j}=X_{j i}$ is a symmetric matrix. Furthermore one has

$$
X_{i j} A_{1}^{i j}=\operatorname{Tr}\left(S^{*} S^{\dagger} S D S^{T}\right)=\operatorname{Tr} D,
$$

which means that the squared term in (6.5) may be written as

$$
\frac{3}{8} e^{3 A}\left|\mathcal{A}^{\prime} X^{i j} \mp \sqrt{2} g A_{1} i j\right|^{2}=\frac{3}{8} e^{3 A}\left|\mathcal{A}^{\prime} \delta_{i j} \mp \sqrt{2} g D_{i j}\right|^{2} .
$$

\subsection{Collecting the boundary terms}

Observe that using the identity [1]

$$
D_{\mu} A_{1}^{i j}=\frac{1}{12 \sqrt{2}}\left(A_{2}{ }^{i} k l m \mathcal{A}_{\mu}{ }^{j k l m}+A_{2}{ }^{j} k l m \mathcal{A}_{\mu}{ }^{i k l m}\right),
$$

the extra terms in (6.5) and (6.6) can be combined to

$$
\pm \frac{g}{4 \sqrt{2}}\left[X^{i j} D_{r}\left(e^{3 A} A_{1 i j}\right)+X_{i j} D_{r}\left(e^{3 A} A_{1}^{i j}\right)\right] .
$$

Using the cyclic properties of the trace, one finds:

$$
\begin{aligned}
X_{i j} \partial_{\mu} A_{1}{ }^{i j} & =\operatorname{Tr}\left[S^{*} S^{\dagger} \partial_{\mu}\left(S D S^{T}\right)\right]=\operatorname{Tr}\left[\left(S^{\dagger} \partial_{\mu} S\right) D+D\left(\partial_{\mu} S^{T}\right) S^{*}+\partial_{\mu} D\right] \\
& =\operatorname{Tr}\left[\partial_{\mu} D+\left(\left(S^{\dagger} \partial_{\mu} S\right)+\left(S^{\dagger} \partial_{\mu} S\right)^{T}\right) D\right] .
\end{aligned}
$$

Note that $\left(S^{\dagger} \partial_{\mu} S\right)+\left(S^{\dagger} \partial_{\mu} S\right)^{T}$ is symmetric and in the Lie algebra of $\mathrm{SU}(8)$. It is therefore purely imaginary and so cancels when added to the complex conjugate:

$$
X^{i j} \partial_{\mu} A_{1 i j}+X_{i j} \partial_{\mu} A_{1}^{i j}=2 \partial_{\mu} \operatorname{Tr} D=2 \partial_{\mu} \operatorname{Tr} \sqrt{A_{1} A_{1}^{\dagger}} .
$$

Finally, there are the connection terms in the covariant derivative:

$$
D_{\mu} A_{1}{ }^{i j}=\partial_{\mu} A_{1}{ }^{i j}-\frac{1}{2} \mathcal{B}_{\mu}{ }^{i}{ }_{k} A_{1}{ }^{k j}-\frac{1}{2} \mathcal{B}_{\mu}{ }^{j}{ }_{k} A_{1}{ }^{i k}=\partial_{\mu} A_{1}{ }^{i j}-\mathcal{B}_{\mu}{ }^{(i}{ }_{k} A_{1}{ }^{j) k},
$$

where we have used the symmetry of $A_{1}{ }^{i j}$. These connection terms yield a contribution:

$$
-X_{i j} \mathcal{B}_{\mu}{ }^{i}{ }_{k} A_{1}{ }^{k j}=-\operatorname{Tr}\left[S^{*} S^{\dagger} \mathcal{B}_{\mu} S D S^{T}\right]=-\operatorname{Tr}\left[\mathcal{B}_{\mu}\left(S D S^{\dagger}\right)\right] .
$$

However, $\left(S D S^{\dagger}\right)$ is hermitian while $\mathcal{B}_{\mu}$ is anti-hermitian and so this trace is purely imaginary and therefore also cancels out when one adds the complex conjugate. This means that with our choice of $X^{i j}$, the $\mathrm{SU}(8)$ connection terms make no contribution to (6.13) and so the complete boundary term may be written as

$$
\pm \frac{g}{2 \sqrt{2}} \partial_{r} \operatorname{Tr}\left[e^{3 A} D\right]= \pm \frac{g}{2 \sqrt{2}} \partial_{r} \operatorname{Tr}\left[e^{3 A} \sqrt{A_{1} A_{1}^{\dagger}}\right] .
$$

Putting this all together, we see that the effective action (6.4) can be written as

$$
\begin{aligned}
S_{\mathrm{B}}=\int d^{3} x d r e^{3 A}[ & \frac{3}{8}\left|\mathcal{A}^{\prime} X_{i j}-\frac{1}{L} A_{1 i j}\right|^{2}-\frac{1}{96}\left|\mathcal{A}_{r}^{i j k l}+\frac{\sqrt{2}}{L} X^{i p} A_{2 p}{ }^{j k l}\right|^{2} \\
& \left.-\frac{1}{96} g^{a b} \mathcal{A}_{a}^{i j k l} \mathcal{A}_{b i j k l}\right]+\left.\frac{1}{4 L} \int d^{3} x e^{3 A} \operatorname{Tr} \sqrt{A_{1} A_{1}^{\dagger}}\right|_{r=r_{0}},
\end{aligned}
$$

where we have explicitly restored the spacetime components, $\mathcal{A}_{a}$ and $\mathcal{A}_{b}$ of $\mathcal{A}_{\mu}, a, b=0,1,2$.

In appendix $\mathrm{C}$ we perform similar computations for consistent truncations of the $\mathcal{N}=8$ theory to reduced levels of supersymmetry. It particular we obtain the analogous form of (6.19) for such truncations. 


\subsection{Asymptotics and counterterms}

We will now argue that for the solutions of interest, namely with

$$
\mathcal{A}(r)=\frac{r}{L}+O\left(e^{-2 r / L}\right),
$$

and (5.10) for the scalars, the two squared terms in the first line in (6.19) obtained by choosing the upper signs in (6.5) and (6.6) vanish at the boundary and that the last term in the bulk integral vanishes as well. This makes the last term in (6.19) a natural candidate for the counterterm. We will further confirm that in section 7 by showing that this boundary counterterm is consistent with the local supersymmetry of the Legendre transformation of the renormalized on-shell action.

Observe that (5.12) implies that the matrix, $\left(A_{1 i j}\right)$, is diagonal to quadratic order in the fields. Using the asymptotic expansion (5.10), we thus have

$$
A_{1 i j}=\delta_{i j}+O\left(e^{-2 r / L}\right), \quad\left(A_{1}^{\dagger} A_{1}\right)_{i j}=\delta_{i j}+O\left(e^{-2 r / L}\right), \quad D_{i j}=\delta_{i j}+O\left(e^{-2 r / L}\right) .
$$

Together with (6.20) and (6.11), where we choose the upper sign, this implies the estimate

$$
\mathcal{A}^{\prime} \delta_{i j}-\sqrt{2} g D_{i j} \sim O\left(e^{-2 r / L}\right) .
$$

Similarly, from (6.21) and the definition (6.7), we find the asymptotic expansion

$$
S^{i}{ }_{j}=S_{0}{ }^{i}{ }_{j}+O\left(e^{-3 r / L}\right),
$$

where $S_{0}$ is a (complex) orthogonal matrix, $S_{0}^{T} S_{0}=1$. Then, cf. (6.9),

$$
X^{i j}=\delta^{i j}+O\left(e^{-3 r / L}\right) .
$$

Once more choosing the upper sign in (6.6) and using (5.11) and (5.13) we find: ${ }^{29}$

$$
\mathcal{A}_{r}{ }^{i j k l}+2 g X^{i m} A_{2 m}{ }^{j k l} \sim O\left(e^{-2 r / L}\right) .
$$

Given the asymptotic expansions (6.22) and (6.25), we see that

$$
\left|\mathcal{A}^{\prime} X_{i j}-\frac{1}{L} A_{1 i j}\right|^{2}, \quad\left|\mathcal{A}_{r}^{i j k l}+\frac{\sqrt{2}}{L} X^{i m} A_{2 m}{ }^{j k l}\right|^{2} \sim \mathcal{O}\left(e^{-4 r / L}\right) .
$$

This shows that the terms in the square bracket in (6.19) vanish at the boundary.

The metric in the boundary directions is $g^{a b}=e^{-2 r / L} \delta^{a b}$ and, from (5.10) and (5.11), one has $\mathcal{A}_{a}{ }^{i j k l} \sim \mathcal{O}\left(e^{-r / L}\right)$ and so the third bulk term in (6.19), including the factor of $e^{3 A}$, vanishes at infinity. Thus even though we allowed scalar fields to depend on the boundary directions, the exponential fall-off of the components of the metric in the boundary directions means that such scalar fluctuations consistently decouples in our effective action near the boundary.

\footnotetext{
${ }^{29}$ Note the order of indices in $(5.11)$.
} 
Thus we are led to the boundary scalar counterterm action:

$$
\begin{aligned}
S_{\mathrm{s}-\mathrm{ct}}= & -\frac{1}{4 L} \int d^{3} x e^{3 r_{0} / L} \operatorname{Tr} \sqrt{A_{1} A_{1}^{\dagger}} \\
=\int d^{3} x e^{3 r_{0} / L}\left[-\frac{2}{L}\right. & -\frac{1}{96 L} \phi_{i j k l} \phi^{i j k l} \\
& \left.\quad+\frac{1}{384 \sqrt{2} L}\left(\phi_{i j k l} \phi_{i j m n} \phi^{k l m n}+\text { c.c. }\right)+\ldots\right] .
\end{aligned}
$$

The combined bulk (6.4) and boundary (6.27) action can be rewitten as:

$$
\begin{aligned}
S_{\mathrm{B}}+S_{\mathrm{s}-\mathrm{ct}}=\int d^{3} x d r e^{3 A}\left[\frac{3}{8}\left|\mathcal{A}^{\prime} X_{i j}-\frac{1}{L} A_{1 i j}\right|^{2}\right. & -\frac{1}{96}\left|\mathcal{A}_{r}{ }^{i j k l}+\frac{\sqrt{2}}{L} X^{i m} A_{2 m}{ }^{j k l}\right|^{2} \\
& \left.-\frac{1}{96} g^{a b} \mathcal{A}_{a}^{i j k l} \mathcal{A}_{b i j k l}\right] .
\end{aligned}
$$

This has a vanishing contribution in the asymptotic region. Put differently, the original bulk action, $S_{\mathrm{B}}$, has divergent and finite pieces at infinity but adding the boundary action, $S_{\text {s-ct }}$, precisely cancels these boundary terms.

Finally, we note that the cubic counterterm in (6.27) depends only on the scalar fields, $\alpha^{i j k l}$. Indeed, it is straightforward to check that for self-dual scalars, $\alpha^{i j k l}$, and anti-self-dual pseudoscalars, $\beta^{i j k l}$,

$$
\alpha^{m n[i j} \alpha^{k l] m n} \quad \text { and } \quad \beta^{m n[i j} \beta^{k l] m n},
$$

are also, respectively, self-dual and anti-selfdual, see appendix D. Thus expanding the cubic counterterm we find

$$
\frac{1}{384 \sqrt{2} L}\left(\phi_{i j k l} \phi^{k l m n} \phi_{m n i j}+\text { c.c. }\right)=\frac{\sqrt{2}}{384 L} \alpha^{i j k l} \alpha^{k l m n} \alpha^{m n i j} .
$$

This is of course in agreement with the branching rules for the $\mathrm{SO}(8)$ tensor products [77]:

$$
35_{i} \otimes 35_{i} \longrightarrow 1+35_{i}+\ldots, \quad 35_{i} \otimes 35_{j} \longrightarrow 35_{k}+\ldots,
$$

and the assignment of $\mathbf{3 5}_{v}$ and $\mathbf{3} \mathbf{5}_{c}$ to the scalars and the pseudoscalars, respectively. Hence the absence of a cubic coupling between the scalars and the pseudoscalars is a consequence of the $\mathrm{SO}(8)$ symmetry.

We should finish this section by emphasizing that while the Bogomolny type argument uses the standard completion of the square that can be used to derive the BPS equations, the latter are not relevant to our focus here. The Bogomolny type argument simply leads to a bulk action with stronger (vanishing) convergence properties at infinity and so can be used to derive the boundary counterterms needed to achieve this outcome.

\section{Boundary sources and $\mathcal{N}=8$ supersymmetry}

\subsection{Preliminaries}

In this section, using methods similar to section 3.5, we show that boundary terms in the supersymmetry variation of the Legendre transformed on-shell action of $\mathcal{N}=8$ supergravity 
are cancelled by the variation of the boundary counterterms,

$$
S_{\mathrm{bdy}}=S_{\mathrm{s}-\mathrm{ct}}+S_{\chi \text {-ct }},
$$

where $S_{\text {s-ct }}$ is the scalar counterterm (6.27) and $S_{\chi \text {-ct }}$ is the gaugino counterterm

$$
S_{\chi-\mathrm{ct}}=\int d^{3} x e^{-3 r_{0}}\left[\frac{1}{24} \bar{\chi}^{i j k} \chi^{i j k}+\text { c.c. }\right] \text {. }
$$

This fermionic counterterm may, at first, seem surprising in that it breaks the $\mathrm{SU}(8)$ symmetry of $\mathcal{N}=8$ supergravity down to $\mathrm{SO}(8)$. Such a symmetry breaking is expected because scalars and pseudoscalars in supergravity are quantized differently.

As in section 3.5, we consider only those variations that involve the scalar and spin$1 / 2$ fields and work in the fixed $\mathrm{AdS}_{4}$ metric background with the corresponding Killing spinors,

$$
\begin{aligned}
\epsilon^{i}(r, \vec{x}) & =e^{r / 2 L} \zeta_{+}{ }^{i}(\vec{x})+e^{-r / 2 L} \zeta_{-}{ }^{i}, & \epsilon_{i}(r, \vec{x}) & =e^{r / 2 L} \zeta_{+i}(\vec{x})+e^{-r / 2 L} \zeta_{-i}, \\
\gamma^{3}{\zeta_{ \pm}}^{i} & =\mp \zeta_{ \pm i}, & \bar{\zeta}_{ \pm}{ }^{i} \gamma^{3} & = \pm \bar{\zeta}_{ \pm i}, \\
\not \partial \zeta_{+}{ }^{i} & =-\frac{3}{L} \zeta_{-i}, & \not \partial \zeta_{+i} & =-\frac{3}{L} \zeta_{-}{ }^{i},
\end{aligned}
$$

obtained by solving the BPS equations (5.21), $\delta \psi_{\mu}{ }^{i}=\delta \psi_{\mu i}=0$, with vanishing scalar fields. However, unlike in section 3, we will use the left- and right-handed spinors rather than the underlying Majorana spinors. This explains why the radiality conditions (7.4) look different from those in (3.24). The two are of course equivalent.

To take advantage of the radiality constraints (7.4) of the Killing spinors, it is convenient to introduce analogous projections of the spin- $1 / 2$ fields. To this end we define

$$
\begin{array}{ll}
\Xi_{i j k}=\frac{1}{2}\left(\chi_{i j k}-\gamma^{3} \chi^{i j k}\right), & \Upsilon_{i j k}=\frac{1}{2}\left(\chi_{i j k}+\gamma^{3} \chi^{i j k}\right), \\
\Xi^{i j k}=\frac{1}{2}\left(\chi^{i j k}-\gamma^{3} \chi_{i j k}\right), & \Upsilon^{i j k}=\frac{1}{2}\left(\chi^{i j k}+\gamma^{3} \chi_{i j k}\right),
\end{array}
$$

where the level of indices indicates the $\gamma^{5}$-chirality. ${ }^{30}$ Then

$$
\gamma^{3} \Xi^{i j k}=-\Xi_{i j k}, \quad \gamma^{3} \Upsilon^{i j k}=\Upsilon_{i j k}
$$

The asymptotic expansions of these fields are given by

$$
\begin{aligned}
& \Xi^{i j k}=e^{-3 r / 2 L} \Xi_{(3 / 2)}{ }^{i j k}+e^{-5 r / 2 L} \Xi_{(5 / 2)}{ }^{i j k}+\ldots, \\
& \Upsilon^{i j k}=e^{-3 r / 2 L} \Upsilon_{(3 / 2)}{ }^{i j k}+e^{-5 r / 2 L} \Upsilon_{(5 / 2)}{ }^{i j k}+\ldots,
\end{aligned}
$$

\footnotetext{
${ }^{30}$ In terms of the underlying Majorana spinors, $\chi_{M}^{i j k}$, the new fields are given by

$$
\Xi^{i j k}=\frac{1}{4}\left(1+\gamma^{5}\right)\left(1-\gamma^{3}\right) \chi_{M}^{i j k}, \quad \Upsilon^{i j k}=\frac{1}{4}\left(1+\gamma^{5}\right)\left(1+\gamma^{3}\right) \chi_{M}^{i j k}, \quad \text { etc. },
$$
}

and hence are chiral projections of the fields with negative/positive radiality, respectively. 
and similarly for the complex conjugate fields. In terms of the leading asymptotic coefficients, the supersymmetry variations (5.20) of the scalar fields become

$$
\begin{aligned}
\delta \alpha_{(1)}{ }^{i j k l}= & 8 \bar{\zeta}_{+}\left[{ }^{i} \Upsilon_{(3 / 2)}{ }^{j k l]}+\frac{1}{3} \eta^{i j k l m n p q} \bar{\zeta}_{+}{ }^{m} \Upsilon_{(3 / 2)}{ }^{n p q},\right. \\
\delta \beta_{(1)}{ }^{i j k l}= & -8 i \bar{\zeta}_{+}{ }^{[i} \Xi_{(3 / 2)}{ }^{j k l]}+\frac{i}{3} \eta^{i j k l m n p q} \bar{\zeta}_{+}{ }^{m} \Xi_{(3 / 2)}{ }^{n p q}, \\
\delta \alpha_{(2)}{ }^{i j k l}= & 8\left(\bar{\zeta}_{-}{ }^{[i} \Xi_{(3 / 2)}{ }^{j k l]}+\bar{\zeta}_{+}\left[{ }^{i} \Upsilon_{(5 / 2)}{ }^{j k l]}\right)\right. \\
& +\frac{1}{3} \eta^{i j k l m n p q}\left(\bar{\zeta}_{-}{ }^{m} \Xi_{(3 / 2)}{ }^{n p q}+\bar{\zeta}_{+}{ }^{m} \Upsilon_{(5 / 2)}{ }^{n p q}\right), \\
\delta \beta_{(2)}{ }^{i j k l}= & -8 i\left(\bar{\zeta}_{-}{ }^{i i} \Upsilon_{(3 / 2)}{ }^{j k l]}+\zeta_{+}{ }^{[i} \Xi_{(5 / 2)}{ }^{j k l]}\right) \\
& +\frac{i}{3} \eta^{i j k l m n p q}\left(\bar{\zeta}_{-}{ }^{m} \Upsilon_{(3 / 2)}{ }^{n p q}+\bar{\zeta}_{+}{ }^{m} \Xi_{(5 / 2)}{ }^{n p q}\right),
\end{aligned}
$$

while the supersymmetry variations (5.22) for the leading modes of the gauginos are

$$
\begin{aligned}
\delta \Xi_{(3 / 2)}{ }^{i j k}=-\frac{2 i}{L} \beta_{(1)}{ }^{i j k l} \zeta_{-}{ }^{l}-\frac{1}{L}[ & \alpha_{(2)}{ }^{i j k l}+\frac{3}{4 \sqrt{2}} \alpha_{(1)}^{m n[i j} \alpha_{(1)}{ }^{k] l m n} \\
& \left.+\frac{3}{4 \sqrt{2}} \beta_{(1)}^{m n[i j} \beta_{(1)}{ }^{k] l m n}-i L \gamma^{3} \not \partial \beta_{(1)}{ }^{i j k l}\right] \zeta_{+}{ }^{l},
\end{aligned}
$$

and

$$
\begin{aligned}
\delta \Upsilon_{(3 / 2)}{ }^{i j k}=\frac{2}{L} \alpha_{(1)}{ }^{i j k l} \zeta_{-}{ }^{l}-\frac{i}{L}[ & \left.-\beta_{(2)}{ }^{i j k l}+\frac{3}{4 \sqrt{2}} \alpha_{(1)}{ }^{m n[i j} \beta_{(1)} k\right] l m n \\
& \left.-\frac{3}{4 \sqrt{2}} \beta_{(1)}^{m n[i j} \alpha_{(1)}^{k] l m n}-i L \gamma^{3} \not \partial \alpha_{(1)}{ }^{i j k l}\right] \zeta_{+}{ }^{l} .
\end{aligned}
$$

The structure of the supersymmetry variations (7.9)-(7.12), modulo the $\mathrm{SO}(8)$ indices, is exactly the same as in (3.26). In particular, we can set the sources:

$$
\beta_{(1)}{ }^{i j k l}(\vec{x})=0, \quad \Xi_{(3 / 2)}{ }^{i j k}(\vec{x})=0,
$$

and

$$
\mathfrak{A}^{i j k l}(\vec{x}) \equiv-\frac{1}{L}\left[\alpha_{(2)}^{i j k l}(\vec{x})+\frac{3}{4 \sqrt{2}} \alpha_{(1)}^{m n[i j}(\vec{x}) \alpha_{(1)}{ }^{k] l m n}(\vec{x})\right],
$$

to zero (cf. (3.28)) consistent with supersymmetry. It follows from (D.6) that $\mathfrak{A}^{i j k l}$ is totally antisymmetric and self-dual. The same calculation as in section 3.6 shows that

$$
\mathfrak{A}^{i j k l}=-\lim _{r \rightarrow \infty} e^{-r / L} \Pi^{i j k l},
$$

where $\Pi^{i j k l}$ is the conjugate momentum

$$
\Pi^{i j k l}=-e^{3 r / L}\left[\partial_{r} \alpha^{i j k l}+\frac{1}{L} \alpha^{i j k l}-\frac{3}{4 \sqrt{2} L} \alpha^{m n[i j} \alpha^{k l] m n}\right],
$$

obtained by varying the bulk plus boundary bosonic action (6.28). Performing the Legendre transform on the scalars amounts then to the addition of

$$
S_{L}=\frac{1}{48} \int d^{3} x \mathfrak{A}^{i j k l}(\vec{x}) \alpha_{(1)}{ }^{i j k l}(\vec{x})
$$

to the action and then extremizing with respect to $\alpha_{(1)}{ }^{i j k l}$. 
The equations of motion for the spin- $1 / 2$ fields that follow from the bulk action (5.16) are:

$$
\gamma^{\mu} D_{\mu} \chi_{i j k}-\frac{1}{12 L} \eta_{i j k p q r l m} A_{2 n}^{p q r} \chi^{l m n}=0
$$

In the $\mathrm{AdS}_{4}$ background,

$$
\gamma^{\mu} D_{\mu}=e^{-r / L} \not \partial+\gamma^{3} \frac{\partial}{\partial r}+\frac{3}{2 L} \gamma^{3}
$$

where $\not \partial$ is the $3 \mathrm{~d}$ Dirac operator along the boundary, and the asymptotic expansion of (7.18) and its complex conjugate equation yield

$$
\begin{aligned}
& \Xi_{(5 / 2)}{ }^{i j k}=-L \not \partial \Upsilon_{(3 / 2) i j k}+\frac{1}{12 \sqrt{2}} \eta_{i j k p q r l m}\left(\alpha_{(1)}{ }^{n p q r} \Xi_{(3 / 2)}^{l m n}-i \beta_{(1)}{ }^{n p q r} \Upsilon_{(3 / 2)}^{l m n}\right), \\
& \Upsilon_{(5 / 2)}{ }^{i j k}=L \not \partial \Xi_{(3 / 2) i j k}-\frac{1}{12 \sqrt{2}} \eta_{i j k p q r l m}\left(\alpha_{(1)}{ }^{n p q r} \Upsilon_{(3 / 2)}^{l m n}-i \beta_{(1)}{ }^{n p q r} \Xi_{(3 / 2)}^{l m n}\right),
\end{aligned}
$$

with arbitrary $\Xi_{(3 / 2)}{ }^{i j k}$ and $\Upsilon_{(3 / 2)}^{i j k}$. This is the $\mathcal{N}=8$ analogue of (3.40).

\subsection{The boundary variation from the bulk $\mathcal{N}=8$ action}

We will now demonstrate explicitly the invariance of the Legendre transformed on-shell renormalized action

$$
\widetilde{S}=S_{\mathrm{bulk}}+S_{\mathrm{bdy}}+S_{L}
$$

under the $\mathcal{N}=8$ superconformal symmetry (7.3). Since the calculation turns out somewhat lengthy, it is helpful to split it into several steps as to make various cancellations more transparent.

We start with the bulk action of the $\mathcal{N}=8$ supergravity, which is known to be invariant — in the bulk — under local supersymmetry variations [1]. However, the proof involves integration by parts which on a space-time with a boundary gives rise to nontrivial boundary terms. A convenient way to identify them is to group individual terms in the variation of the bulk supergravity Lagrangian, $\mathcal{L}_{\text {bulk }}$, according to whether they contain derivatives of the supersymmetry parameters or not. Schematically, we may write this as

$$
\delta \mathcal{L}_{\text {bulk }}=\bar{V}_{i} \epsilon^{i}+\bar{X}_{i}^{\mu} D_{\mu} \epsilon^{i}+\text { c.c. } .
$$

The invariance of the action in the bulk means that after integration by parts,

$$
\left(\bar{V}_{i}-D_{\mu} \bar{X}_{i}^{\mu}\right) \epsilon^{i}+\text { c.c. }=0 .
$$

In the $\mathrm{AdS}_{4}$ background that we are considering, the remaining boundary terms are thus given by

$$
\delta S_{\mathrm{bulk}}=\int d^{3} x e^{3 r_{0} / L}\left[\bar{X}^{3}{ }_{i} \epsilon^{i}+\text { c.c. }\right] .
$$

In practice, this means that in order to extract the boundary terms of interest, we must look only at those terms in the bulk action that upon the supersymmetry variation give rise 
to derivatives of the supersymmetry parameters. There are three sources of such terms: the kinetic terms with derivatives of the varied fields, the Noether coupling of the gravitino to the supersymmetry current that is independent of $g$, and the additional Noether coupling due to gauging.

Since we only are interested in the variations that contain the scalar fields and the gauginos, we must consider only the following terms in the bulk action:

$$
\begin{aligned}
S_{\text {bulk }}=\int d^{4} x & \sqrt{-g}\left[-\frac{1}{96} \mathcal{A}_{\mu}{ }^{i j k l} \mathcal{A}^{\mu}{ }_{i j k l}-\frac{1}{12}\left(\bar{\chi}^{i j k} \gamma^{\mu} D_{\mu} \chi_{i j k}+\bar{\chi}_{i j k} \gamma^{\mu} D_{\mu} \bar{\chi}^{i j k}\right)\right. \\
& \left.-\frac{1}{12}\left(\mathcal{A}_{\mu}{ }^{i j k l} \bar{\chi}_{i j k} \gamma^{\nu} \gamma^{\mu} \psi_{\nu l}+\text { c.c. }\right)+\frac{g}{6}\left(A_{2}{ }^{i}{ }_{j k l} \bar{\psi}_{\mu i} \gamma^{\mu} \chi^{j k l}+\text { c.c. }\right)\right] .
\end{aligned}
$$

Then, using the supersymmetry variations (5.21), (5.22) and (5.18), we find essentially by inspection that the boundary terms in the variation of the bulk action are given by

$$
\begin{aligned}
\bar{\epsilon}_{j} X^{\mu j}=- & \frac{1}{6} \mathcal{A}^{\mu i j k l} \bar{\epsilon}_{i} \chi_{j k l}+\frac{1}{12} A_{\nu}{ }^{i j k l} \bar{\epsilon}_{l} \gamma^{\nu} \gamma^{\mu} \chi_{i j k}-\frac{g}{6} A_{2}{ }^{l}{ }_{i j k} \bar{\epsilon}_{l} \gamma^{\mu} \chi^{i j k} \\
& +\frac{1}{6} \mathcal{A}_{\nu}{ }^{i j k l} \bar{\epsilon}_{i} \gamma^{\nu} \gamma^{\mu} \chi_{j k l}+\frac{g}{3} A_{2}{ }^{i}{ }_{j k l} \bar{\epsilon}_{i} \gamma^{\mu} \chi^{j k l}
\end{aligned}
$$

plus its complex conjugate, $\bar{\epsilon}^{i} X^{\mu}{ }_{i}$. We have kept here the same order of terms as in (7.25) to indicate the origin of each term. Note that the variation of the gaugino kinetic action (the second and third terms in (7.26)) is proportional to the variation of the Noether coupling in the last two terms in (7.26). Hence ${ }^{31}$

$$
\begin{aligned}
\bar{\epsilon}_{j} X^{\mu j}+\text { c.c. } & =-\frac{1}{6} \mathcal{A}^{\mu i j k l} \bar{\epsilon}_{i} \chi_{j k l}+\frac{1}{12} \mathcal{A}_{\nu}{ }^{i j k l} \bar{\epsilon}_{i} \gamma^{\nu} \gamma^{\mu} \chi_{j k l}+\frac{g}{6} A_{2}{ }^{i}{ }_{j k l} \bar{\epsilon}_{i} \gamma^{\mu} \chi^{j k l}+\text { c.c. }, \\
& =-\frac{1}{6} \mathcal{A}^{\mu i j k l} \bar{\epsilon}_{i} \chi_{j k l}-\frac{1}{12} \delta \bar{\chi}_{j k l} \gamma^{\mu} \chi^{j k l}+\text { c.c. }
\end{aligned}
$$

where in the second line we used (5.22).

\subsection{Adding counterterms}

The first term in the second line of (7.27) is the only boundary contribution to (7.24) from the variation of the bulk bosonic action (5.7), that is

$$
\delta S_{\mathrm{B}}=\int d^{3} x e^{3 r_{0} / L}\left[-\frac{1}{6} \mathcal{A}^{\mu i j k l} \bar{\epsilon}_{i} \chi_{j k l}+\text { c.c. }\right] .
$$

We will first show that modulo source terms on the boundary, both the infinite and finite terms in (7.28) are cancelled by the variation of the bosonic counterterms in (6.27):

$$
\begin{aligned}
\delta S_{\mathrm{s}-\mathrm{ct}}=\int d^{3} x e^{3 r_{0} / L}\left[-\frac{c_{2}}{48 L}\right. & \left(\alpha^{i j k l} \delta \alpha^{i j k l}+\beta^{i j k l} \delta \beta^{i j k l}\right) \\
& \left.+\frac{c_{3}}{128 \sqrt{2} L} \alpha^{i j k l} \alpha^{k l m n} \delta \alpha^{i j m n}\right] .
\end{aligned}
$$

\footnotetext{
${ }^{31}$ To properly understand the signs, note the positions of the contracted indices $i j k l$ in the second a fourth terms in (7.26): these two terms differ by a factor of -2 just like the third and fifth terms.
} 
To keep track of the origin of various terms, we have introduced here constants $c_{2}$ and $c_{3}$, to be set $c_{2}=c_{3}=1$ afterwards.

The expansion of (7.28) and (7.29) yields the following result: ${ }^{32}$

$$
\begin{aligned}
(7.28)+(7.29)= & \frac{1}{3 L} \int d^{3} x e^{r_{0} / L}\left(1-c_{2}\right)\left[\alpha_{(1)}{ }^{i j k l} \bar{\zeta}_{+}{ }^{i} \Upsilon_{(3 / 2)}{ }^{j k l}+i \beta_{(1)}{ }^{i j k l} \bar{\zeta}_{+}{ }^{i} \Xi_{(3 / 2)}{ }^{j k l}\right] \\
& +\frac{1}{3 L} \int d^{3} x\left(1-c_{2}\right)\left[\alpha_{(1)}{ }^{i j k l} \bar{\zeta}_{+}{ }^{i} \Upsilon_{5 / 2}{ }^{j k l}+i \beta_{(1)}{ }^{i j k l} \bar{\zeta}_{+}{ }^{i} \Xi_{5 / 2}{ }^{j k l}\right. \\
& \left.+\alpha_{(1)}{ }^{i j k l} \bar{\zeta}_{-}{ }^{i} \Xi_{(3 / 2)}{ }^{j k l}+i \beta_{(1)}{ }^{i j k l} \bar{\zeta}_{-}{ }^{i} \Upsilon_{(3 / 2)}{ }^{j k l}\right] \\
& +\frac{1}{3 L} \int d^{3} x\left[\left(\left(2-c_{2}\right) \alpha_{(2)}{ }^{i j k l}+\frac{3 c_{3}}{4 \sqrt{2}} \alpha_{(1)}^{i j m n} \alpha_{(1)}{ }^{k l m n}\right) \zeta_{+}{ }^{i} \Upsilon_{(3 / 2)}{ }^{j k l}\right. \\
& \left.+\left(2-c_{2}\right) i \beta_{(2)}{ }^{i j k l} \zeta_{+}{ }^{i} \Xi_{(3 / 2)}{ }^{j k l}\right] .
\end{aligned}
$$

This shows that the quadratic counterterm removes the divergence at the boundary as well as it cancels a number of finite terms given by the second integral. After using (7.9), (7.14) and (7.17), the remaining terms are

$$
\delta S_{\mathrm{B}}+\delta S_{\mathrm{s}-\mathrm{ct}}=\int d^{3} x\left[-\frac{1}{48} \mathfrak{A}^{i j k l} \delta \alpha_{(1)}{ }^{i j k l}+\frac{i}{3 L} \beta_{(2)}{ }^{i j k l} \zeta_{+}{ }^{i} \Xi_{(3 / 2)}{ }^{j k l}\right],
$$

and they indeed vanish in the absence of sources, cf. (7.13) and (7.14).

Next consider the second boundary term in the variation of the bulk action (7.27) and combine it with the variation of the gaugino counterterm (7.2) multiplied by an overall constant, $c_{\chi}$. In terms of the modes (7.8), we then have

$$
\begin{aligned}
& \int d^{3} x e^{3 r_{0} / L}\left[-\frac{1}{12} \delta \bar{\chi}^{i j k} \gamma^{3} \chi^{i j k}+\frac{c_{\chi}}{12} \delta \bar{\chi}^{i j k} \chi^{i j k}+\text { c.c. }\right] \\
& =\int d^{3} x\left[-\frac{1}{12}\left(1-c_{\chi}\right) \delta \bar{\Xi}_{(3 / 2)}^{i j k} \Upsilon_{(3 / 2)}^{i j k}+\frac{1}{12}\left(1+c_{\chi}\right) \delta \bar{\Upsilon}_{(3 / 2)}^{i j k} \Xi_{(3 / 2)}^{i j k}+\text { c.c. }\right] .
\end{aligned}
$$

Examining the variations (7.11) and (7.12), it is clear that we can cancel the second term in (7.31) only by setting $c_{\chi}=1$. Substituting (7.12) in (7.32) and then using (D.8) and the radialities (7.4) and (7.7), we find

$$
\begin{aligned}
\frac{1}{6} \delta \bar{\Upsilon}_{(3 / 2)}{ }^{i j k} \Xi_{(3 / 2)}{ }^{i j k}+\text { c.c. }= & -\frac{2}{3 L} \alpha_{(1)}{ }^{i j k l} \bar{\zeta}_{-}^{i} \Xi_{(3 / 2)}{ }^{j k l}-\frac{1}{3} \zeta_{+i} \not \partial \alpha_{(1)}{ }^{i j k l} \Xi_{(3 / 2)}{ }^{j k l} \\
& -\left(\frac{i}{2 \sqrt{2}} \alpha_{(1)}{ }^{i j m n} \beta_{(1)}{ }^{k l m n}+\frac{i}{3 L} \beta_{(2)}{ }^{i j k l}\right) \zeta_{+}{ }^{i} \Xi_{(3 / 2)}{ }^{j k l} .
\end{aligned}
$$

The variation of the last term in (7.21) is

$$
\delta S_{L}=\int d^{3} x\left[\frac{1}{48} \mathfrak{A}^{i j k l} \delta \alpha_{(1)}^{i j k l}+\frac{1}{48} \alpha_{(1)}^{i j k l} \delta \mathfrak{A}^{i j k l}\right],
$$

\footnotetext{
${ }^{32}$ Note that we consider here only a subset of terms from the full supersymmetry variation of the bosonic action and hence there is no contradiction with the result of the asymptotic analysis in section 6.5, namely that the renormalized bosonic action vanishes at the boundary.
} 
where the first term cancels against the first term in (7.31). To complete the proof of invariance, we must show that the second term in (7.34) combines with the first three terms in (7.33) into a total derivative along the boundary.

From (7.14), (7.9), (7.10) and using the identities (D.6), (D.8), (D.9) and (D.10) in appendix D, we have

$$
\begin{aligned}
\frac{1}{48} \alpha_{(1)}{ }^{i j k l} \delta \mathfrak{A}^{i j k l}= & -\frac{1}{3 L} \alpha_{(1)}{ }^{i j k l}\left(\bar{\zeta}_{-}{ }^{i} \Xi_{(3 / 2)}{ }^{j k l}+\bar{\zeta}_{+}{ }^{i} \Upsilon_{(5 / 2)}{ }^{j k l}\right) \\
& -\frac{1}{2 \sqrt{2} L} \alpha_{(1)}{ }^{i j m n} \alpha_{(1)}{ }^{k l m n} \bar{\zeta}_{-}{ }^{i} \Upsilon_{(3 / 2)}{ }^{j k l} \\
= & -\frac{1}{3 L} \alpha_{(1)}{ }^{i j k l} \bar{\zeta}_{-}{ }^{i} \Xi_{(3 / 2)}{ }^{j k l}+\frac{i}{2 \sqrt{2}} \alpha_{(1)}{ }^{i j m n} \beta_{(1)}{ }^{k l m n} \zeta_{+}{ }^{i} \Xi_{(3 / 2)}{ }^{j k l} \\
& -\frac{1}{3} \alpha_{(1)}{ }^{i j k l} \bar{\zeta}_{+}{ }^{i} \not \partial \Xi_{(3 / 2) j k l},
\end{aligned}
$$

where in the second step we have also used the fermion equations of motion (7.20) to eliminte $\Upsilon_{(5 / 2)}{ }^{j k l}$. Adding the variations in (7.31), (7.33) and (7.35) we are left with

$$
\begin{aligned}
\delta \widetilde{S} & =\int d^{3} x\left[-\frac{1}{L} \alpha_{(1)}{ }^{i j k l} \bar{\zeta}_{-}{ }^{i} \Xi_{(3 / 2)}{ }^{j k l}-\frac{1}{3} \zeta_{+i} \not \partial \alpha_{(1)}{ }^{i j k l} \Xi_{(3 / 2)}{ }^{j k l}-\frac{1}{3} \alpha_{(1)}{ }^{i j k l} \bar{\zeta}_{+}{ }^{i} \not \partial \Xi_{(3 / 2) j k l}\right] \\
& =\int d^{3} x\left[-\frac{1}{3} \frac{\partial}{\partial x^{a}}\left(\alpha_{(1)}{ }^{i j k l} \zeta_{+i} \gamma^{a} \Xi_{(3 / 2)}{ }^{j k l}\right)\right]
\end{aligned}
$$

which vanishes. This concludes the proof of invariance.

\section{2 - and 3-point correlators from $\mathcal{N}=8$ supergravity}

\subsection{The counterterms in the $\mathrm{SL}(8, \mathbb{R})$ basis}

To calculate the three point functions of the operators, $\mathcal{O}_{I J}$, we first transform the scalar fields from the $\mathrm{SU}(8)$ to the $\mathrm{SL}(8, \mathbb{R})$ basis [66]. This replaces the antisymmetric self-dual tensor, $\alpha^{i j k l}$, by the symmetric traceless tensor, $A^{I J}$,

$$
\alpha^{i j k l}=\frac{1}{4}\left(\Gamma_{I K}\right)^{i j}\left(\Gamma_{J K}\right)^{k l} A^{I J}, \quad A^{I J}=\frac{1}{96}\left(\Gamma_{I K}\right)^{i j}\left(\Gamma_{J K}\right)^{k l} \alpha^{i j k l},
$$

and the anti-self-dual tensor, $\beta^{i j k l}$, by the self-dual tensor, $B^{I J K L}$,

$$
\beta^{i j k l}=\frac{1}{16}\left(\Gamma_{I J}\right)^{i j}\left(\Gamma_{K L}\right)^{k l} B^{I J K L}, \quad B^{I J K L}=\frac{1}{16}\left(\Gamma_{I J}\right)^{i j}\left(\Gamma_{K L}\right)^{k l} \beta^{i j k l},
$$

where $I, J, \ldots$ indices lie in $\boldsymbol{8}_{v}$ and $\Gamma_{I J}$ are chiral $\mathrm{SO}(8)$ generators. In terms of the new fields, the bulk action (5.23) and the boundary counterterms (6.27) read:

$$
\begin{aligned}
& S_{\mathrm{B}}=\int d^{4} x \sqrt{-g}[ \frac{1}{2} R-\frac{1}{4} \partial_{\mu} A^{I J} \partial^{\mu} A^{I J}-\frac{1}{96} \partial_{\mu} B^{I J K L} \partial^{\mu} B^{I J K L} \\
&\left.+\frac{1}{2 L^{2}}\left(6+A^{I J} A^{I J}+\frac{1}{24} B^{I J K L} B^{I J K L}\right)\right], \\
& S_{\text {s-ct }}=\int d^{3} x e^{3 r_{0} / L}\left[-\frac{2}{L}-\frac{1}{4 L} A^{I J} A^{I J}+\frac{1}{6 \sqrt{2} L} A^{I J} A^{J K} A^{K I}\right] .
\end{aligned}
$$


The source field (7.14) becomes

$$
\mathfrak{A}^{I J}=-\frac{1}{L}\left[A_{(2)}^{I J}+\frac{1}{\sqrt{2}}\left(A_{(1)}^{I K} A_{(1)}^{J K}-\frac{1}{8} \delta^{I J} A_{(1)}{ }^{M N} A_{(1)}{ }^{M N}\right)\right],
$$

as can be verified by calculating the momentum $\Pi^{I J}$ from the action (8.3)-(8.4).

\subsection{The correlators of the operators $\mathcal{O}_{I J}(\vec{x})$}

Finally we calculate the 2- and 3-point functions of the 35 operators $\mathcal{O}_{I J}(\vec{x})$ with scale dimension $\Delta=1$. This computation parallels the one in section 4 , the only difference being that here we need to carefully keep track of the $\mathrm{SO}(8)$ vector indices. Just as in section 4 , let us set $L=1$. We will reinstate $L$ by dimensional analysis at the end.

The starting point is the action (8.3)-(8.4) with the pseudoscalars set to zero. In Euclidean signature, it reads

$$
\begin{aligned}
S= & \frac{1}{\kappa^{2}} \int d^{4} x \sqrt{g}\left[\frac{1}{4} \partial_{\mu} A^{I J} \partial^{\mu} A^{I J}-\frac{1}{2} A^{I J} A^{I J}\right] \\
& +\frac{1}{\kappa^{2}} \int d^{3} x e^{3 r_{0}}\left[\frac{1}{4} A^{I J} A^{I J}-\frac{1}{6 \sqrt{2}} A^{I J} A^{J K} A^{K I}\right]+O\left(A^{4}\right),
\end{aligned}
$$

where we restored the factor of $1 / \kappa^{2}$ that accounts for a proper normalization of the Einstein-Hilbert term in (8.3). As in (4.3), we expand $A^{I J}$ as

$$
A^{I J}(r, \vec{x})=e^{-r} A_{(1)}^{I J}(\vec{x})+e^{-2 r} A_{(2)}^{I J}(\vec{x})+\cdots,
$$

and we can write the on-shell action as a simple generalization of (4.5):

$$
\begin{aligned}
S_{\text {on-shell }}\left[A_{(1)}^{I J}\right]= & -\frac{1}{4 \kappa^{2}} \int d^{3} x d^{3} y \frac{A_{(1)}^{I J}(\vec{x}) A_{(1)}^{I J}(\vec{y})}{\pi^{2}|\vec{x}-\vec{y}|^{4}} \\
& -\frac{1}{6 \sqrt{2} \kappa^{2}} \int d^{3} x A_{(1)}^{I J}(\vec{x}) A_{(1)}^{J K}(\vec{x}) A_{(1)}^{K I}(\vec{x})+O\left(A_{(1)}^{4}\right) .
\end{aligned}
$$

To obtain the generating functional of connected correlators of $\mathcal{O}_{I J}(\vec{x})$, we should pass to the Legendre transform of (8.8):

$$
\tilde{S}_{\text {on-shell }}\left[\mathfrak{A}^{I J}\right]=S_{\text {on-shell }}\left[A_{(1)}^{I J}\right]+\frac{1}{2 \kappa^{2}} \int d^{3} x \mathfrak{A}^{I J}(\vec{x}) A_{(1)}^{I J}(\vec{x}),
$$

computed after extremizing the right-hand side with respect to $A_{(1)}^{I J}(\vec{x})$. By analogy with (4.7), this extremization gives

$$
\mathfrak{A}^{I J}(\vec{x})=\frac{1}{\pi^{2}} \int d^{3} y \frac{A_{(1)}^{I J}(\vec{y})}{|\vec{x}-\vec{y}|^{4}}-\frac{1}{\sqrt{2}}\left[A_{(1)}^{J K}(\vec{x}) A_{(1)}^{K I}(\vec{x})-\frac{1}{8} \delta^{I J} A_{(1)}^{K L}(\vec{x}) A_{(1)}^{K L}(\vec{x})\right]+O\left(A_{(1)}^{3}\right) .
$$

Repeating the steps that led to (4.11), we obtain

$$
\begin{aligned}
\tilde{S}_{\text {on-shell }}\left[\mathfrak{A}^{I J}\right]= & -\frac{1}{8 \pi^{2} \kappa^{2}} \int d^{3} x d^{3} y \frac{\mathfrak{A}^{I J}(\vec{x}) \mathfrak{A}^{I J}(\vec{y})}{|\vec{x}-\vec{y}|^{2}} \\
& +\frac{1}{6 \sqrt{2} L \kappa^{2}} \int d^{3} x d^{3} y d^{3} z \frac{\mathfrak{A}^{I J}(\vec{x}) \mathfrak{A}^{J K}(\vec{y}) \mathfrak{A}^{K I}(\vec{z})}{8 \pi^{3}|\vec{x}-\vec{y}||\vec{y}-\vec{z}||\vec{x}-\vec{z}|}+O\left(\mathfrak{A}^{4}\right),
\end{aligned}
$$

where we restored the appropriate factors of $L$. 
To identify $-\tilde{S}_{\text {on-shell }}\left[\mathfrak{A}^{I J}\right]$ with the generating functional of connected correlators of $\mathcal{O}_{I J}(\vec{x})$, we should account for the fact that $\mathfrak{A}^{I J}(\vec{x})$ may not be precisely the field theory source for $\mathcal{O}_{I J}(\vec{x})$, but it might differ from it by a constant,

$$
\text { Source for } \mathcal{O}_{I J}(\vec{x})=\frac{\mathcal{C}}{L} \mathfrak{A}^{I J}(\vec{x}),
$$

with $\mathcal{C}$ being a dimensionless constant, and a factor of $1 / L$ being required by dimensional analysis. Adjusting for the proportionality constant in (8.12), we have from (8.11) that

$$
\begin{aligned}
\left\langle\mathcal{O}_{I J}\left(\vec{x}_{1}\right) \mathcal{O}_{I J}\left(\vec{x}_{2}\right)\right\rangle & =\frac{L^{2}}{16 \pi^{3} G_{4} \mathcal{C}^{2}} \frac{1}{\left|\vec{x}_{1}-\vec{x}_{2}\right|^{2}}, \\
\left\langle\mathcal{O}_{I J}\left(\vec{x}_{1}\right) \mathcal{O}_{J K}\left(\vec{x}_{2}\right) \mathcal{O}_{K I}\left(\vec{x}_{3}\right)\right\rangle & =-\frac{L^{2}}{64 \sqrt{2} \pi^{4} G_{4} \mathcal{C}^{3}} \frac{1}{\left|\vec{x}_{1}-\vec{x}_{2}\right|\left|\vec{x}_{1}-\vec{x}_{2}\right|\left|\vec{x}_{2}-\vec{x}_{3}\right|},
\end{aligned}
$$

with no sum over $I, J$, and $K$. In the index free " $M$ notation," these expressions become

$$
\begin{aligned}
\left\langle\mathcal{O}\left(\vec{x}_{1}, M_{1}\right) \mathcal{O}\left(\vec{x}_{2}, M_{2}\right)\right\rangle & =\frac{L^{2}}{8 \pi^{3} G_{4} \mathcal{C}^{2}} \frac{\operatorname{tr}\left(M_{1} M_{2}\right)}{\left|\vec{x}_{1}-\vec{x}_{2}\right|^{2}}, \\
\left\langle\mathcal{O}\left(\vec{x}_{1}, M_{1}\right) \mathcal{O}\left(\vec{x}_{2}, M_{2}\right) \mathcal{O}\left(\vec{x}_{3}, M_{3}\right)\right\rangle & =-\frac{L^{2}}{16 \sqrt{2} \pi^{4} G_{4} \mathcal{C}^{3}} \frac{\operatorname{tr}\left(M_{1} M_{2} M_{3}+M_{1} M_{3} M_{2}\right)}{\left|\vec{x}_{1}-\vec{x}_{2}\right|\left|\vec{x}_{1}-\vec{x}_{2}\right|\left|\vec{x}_{2}-\vec{x}_{3}\right|} .
\end{aligned}
$$

The relations (8.14) are in complete agreement with the field theory results of section 2 ! Indeed, these relations imply

$$
c_{2}=\frac{L^{2}}{8 \pi^{3} G_{4} \mathcal{C}^{2}}, \quad c_{3}=-\frac{L^{2}}{16 \sqrt{2} \pi^{4} G_{4} \mathcal{C}^{3}},
$$

where $c_{2}$ and $c_{3}$ are as in (2.12). It is straightforward to see that the ratio $c_{3}^{2} / c_{2}^{3}$, which is independent of the normalization constant $\mathcal{C}$ agrees with the result (2.23) provided that we use $c_{T}=32 L^{2} /\left(\pi G_{4}\right)$ as in (2.26). Moreover, we see that if we work with operators $\mathcal{O}_{I J}$ that are canonically normalized in the sense explained in section 2 , for which the 2 - and 3 -point functions are given in (2.27), we have

$$
\mathcal{C}=-\frac{1}{\sqrt{2}}
$$

Up to an overall sign, this normalization constant could have also been inferred from [14].

\section{Conclusions}

The goal of this paper was to resolve a puzzle concerning the 3-point functions of dimension-1 scalar operators in 3d supersymmetric CFTs with gravity duals. In the case of the $\mathcal{N}=8$ ABJM theory at Chern-Simons level $k=1,2$, one can calculate this 3 -point function exactly using the method of supersymmetric localization. It does not vanish. When $k=1$, the gravity dual of $\mathcal{N}=8 \mathrm{ABJM}$ is $11 \mathrm{~d}$ supergravity on $\mathrm{AdS}_{4} \times S^{7}$. The $4 \mathrm{~d}$ maximally supersymmetric gauged $\mathrm{SO}(8)$ supergravity theory captures the dynamics of the gravity multiplet in which the superconformal primaries are a $\mathbf{3 5}_{v}$ of scalar fields $A^{I J}$ 
dual to the field theory operators $\mathcal{O}_{I J}$ of dimension 1 . However the bulk action contains no cubic couplings of the $A^{I J}$, so the traditional calculation of holographic 3-point functions is not applicable.

The resolution is that the supergravity theory requires cubic boundary terms that provide precisely the right interactions to reproduce the boundary 3-point functions. Our main result (8.15) obtained from holography agrees precisely with the field theory expectation (2.23). The boundary terms were first motivated by a Bogomolny argument for BPS domain walls. They were then derived more rigorously by requiring that the total derivatives usually neglected in supersymmetry variations of an action are cancelled by boundary counter terms that include the necessary cubic.

Bulk fields dual to dimension-1 scalar operators in a 3d CFT enjoy alternate quantization as prescribed in [25]. The generating functional for their correlators is the Legendre transform of the renormalized on-shell action that includes the new cubic boundary term. The supersymmetry properties of the renormalized on-shell action and its Legendre transform are as follows:

1. When sources are absent the on-shell action is invariant and the effect of the cubic term is to produce nonlinear boundary conditions on the bulk fields. Naive boundary conditions would break supersymmetry.

2. Sources are needed to calculate correlation functions. The sources and their supersymmetry transformations are determined from the near-boundary asymptotics of the bulk fields. When sources are included, only the Legendre transform is invariant.

Independent of supersymmetry, the Legendre transform plays a crucial role in the calculation of the 3-point function $\left\langle\mathcal{O}_{I J}\left(\vec{x}_{1}\right) \mathcal{O}_{J K}\left(\vec{x}_{2}\right) \mathcal{O}_{K I}\left(\vec{x}_{3}\right)\right\rangle$. This is developed in an $\mathcal{N}=1$ toy model in section 4 and extended to $\mathcal{N}=8$ supergravity in section 8 . The argument is both intricate and elegant, and gives considerable insight into the working of the Legendre transform.

In the general framework of field theories with boundaries, the condition for a boundary to preserve a conserved charge of the bulk theory is very simple: in the absence of boundary sources, there must be no net flux of the conserved charge across the boundary. In particular for supersymmetric theories, if there are no boundary sources, then flux of the supercurrent across the boundary should be zero. The supersymmetric Noether currents of the $\mathcal{N}=8$ (global) supersymmetries are:

$$
\mathcal{J}_{\mu}{ }^{i} \equiv \frac{1}{6} \mathcal{A}_{\nu}{ }^{i j k l} \gamma^{\nu} \gamma_{\mu} \chi_{j k l}+\frac{g}{3} A_{2}{ }^{i}{ }_{j k l} \gamma_{\mu} \chi^{j k l}
$$

and so supersymmetric boundary conditions should imply no leakage of supercharge at infinity:

$$
\int d^{3} x e^{3 A}\left(\bar{\epsilon}_{i} \mathcal{J}_{r}^{i}+\text { c.c. }\right) \quad \longrightarrow \quad 0, \quad r \rightarrow \infty .
$$

It is relatively straightforward to establish that this indeed is a consequence of the vanishing of (7.13) and (7.14) and similarly, for the $\mathcal{N}=1$ theory, with the boundary conditions (3.28). 


\section{Acknowledgments}

DZF thanks Massimo Bianchi and Emery Sokatchev for useful suggestions and discussions. KP and NPW would also like to thank Nikolay Bobev, Sergei Gukov and Vyacheslav Lysov for discussions that led to KP's and NPW's interest in the issues considered in this paper. The interest of DZF, SSP and NPW was stimulated by discussions at a workshop organized by the Michigan Center for Theoretical Physics. DZF and KP thank the Galileo Galilei Institute for Theoretical Physics for the hospitality and the INFN for partial support during the completion of this work. SSP thanks Simone Giombi, Zohar Komargodski, Andy Stergiou, and Ran Yacoby for useful discussions. DZF was supported in part by the US NSF Grant No. PHY 1620045 and as Templeton Visiting Professor at Stanford. The work of KP and NPW was supported in part by DOE grant DE-SC0011687. SSP was supported by the US NSF under Grant No. PHY-1418069.

\section{A An alternative computation of 3-point functions of dimension-1 operators}

Here we present an alternative method of computing 3-point functions of dimension-1 scalar operators that can be used in SCFTs with extended supersymmetry. As mentioned in section 2 , in $\mathcal{N}=2$ SCFTs, 2-point functions of dimension-1 scalars in flavor current multiplets can be computed via supersymmetric localization by taking two derivatives of the $S^{3}$ free energy. Indeed, given a flat space $\mathcal{N}=2$ SCFT with R-symmetry current $j_{R}^{\mu}$ and Abelian flavor symmetries generated by $j_{(\alpha)}^{\mu}$, one can construct [17] a unique supersymmetric theory on $S^{3}$ that is invariant under $\mathrm{SU}(2 \mid 1)_{\ell} \times \mathrm{SU}(2)_{r}$, whose bosonic part consists of the $\mathrm{SU}(2)_{\ell} \times \mathrm{SU}(2)_{r}$ isometry group of $S^{3}$ as well as a $\mathrm{U}(1)$ symmetry generated by

$$
j_{R}^{\mu}+\sum_{\alpha} t_{\alpha} j_{(\alpha)}^{\mu}
$$

where $t_{\alpha}$ are parameters. Using the technique of supersymmetric localization, one can moreover compute the $S^{3}$ free energy $F\left(t_{\alpha}\right)$ of this theory [17]. One then has [45]

$$
\left\langle J_{(\alpha)}\left(\vec{x}_{1}\right) J_{(\beta)}\left(\vec{x}_{2}\right)\right\rangle=-\frac{2}{\pi^{2}}\left(\left.\frac{\partial^{2} F}{\partial t^{\alpha} \partial t^{\beta}}\right|_{t_{\alpha}=0}\right) \frac{1}{(4 \pi)^{2}\left|\vec{x}_{1}-\vec{x}_{2}\right|^{2}},
$$

where $J_{(\alpha)}$ are the dimension-1 scalars in the conserved current multiplets, normalized as in (2.4).

As we now argue, the 3 -point function of $J_{(\alpha)}$ can also be computed from $F\left(t_{\alpha}\right)$ via

$$
\left\langle J_{(\alpha)}\left(\vec{x}_{1}\right) J_{(\beta)}\left(\vec{x}_{2}\right) J_{(\gamma)}\left(\vec{x}_{3}\right)\right\rangle=\frac{1}{\pi^{2}}\left(\left.\frac{\partial^{3} F}{\partial t^{\alpha} \partial t^{\beta} \partial t^{\gamma}}\right|_{t_{\alpha}=0}\right) \frac{1}{(4 \pi)^{3}\left|\vec{x}_{1}-\vec{x}_{2}\right|\left|\vec{x}_{1}-\vec{x}_{3}\right|\left|\vec{x}_{2}-\vec{x}_{3}\right|},
$$

but only in SCFTs that have at least $\mathcal{N}=4$ supersymmetry, and where at least two of these $\mathcal{N}=2$ flavor current multiplets descend from half-BPS multiplets of the ex-

tended supersymmetry. Indeed, $\left.\frac{\partial^{3} F}{\partial t^{\alpha} \partial t^{\beta} \partial t^{\gamma}}\right|_{t_{\alpha}=0}$ is proportional to the 3-point function 
$\left\langle J_{(\alpha)}\left(\vec{x}_{1}\right) J_{(\beta)}\left(\vec{x}_{2}\right) J_{(\gamma)}\left(\vec{x}_{3}\right)\right\rangle$ whenever all 3-point functions of the operators multiplying $t_{\alpha}$ in the $S^{3}$ action of [17] are proportional to $\left\langle J_{(\alpha)}\left(\vec{x}_{1}\right) J_{(\beta)}\left(\vec{x}_{2}\right) J_{(\gamma)}\left(\vec{x}_{3}\right)\right\rangle$. This is true when at least two of the $\mathcal{N}=2$ flavor current multiplets descend from half-BPS multiplets of the extended supersymmetry, because in this case there is only one superspace invariant that gives the 3-point function of the extended supersymmetry multiplets. A free theory computation then gives the proportionality constant in (A.3).

We now show how (A.2) and (A.3) work in $\mathrm{U}(N)_{k} \times \mathrm{U}(N)_{-k}$ ABJM theory [3], first when $N=1$ where the theory is free, and afterwards in the large- $N$ limit where the theory has a holographic dual. We will be primarily interested in taking $k=1$ or 2 where supersymmetry is enhanced to $\mathcal{N}=8$. Recall that in $\mathcal{N}=2$ notation, ABJM theory has 2 vector multiplets with Chern-Simons levels $(k,-k)$, two bi-fundamental chiral multiplets $\mathcal{Z}_{a}, a=1,2$ transforming in $(\mathbf{N}, \overline{\mathbf{N}})$ of $\mathrm{U}(N) \times \mathrm{U}(N)$, and two bi-fundamental chiral multiplets $\mathcal{W}_{a}, a=1,2$ transforming in the conjugate representation of the gauge group. Due to the extended supersymmetry, the R-charges of these chiral multiplets take the free field value $1 / 2$.

There are 3 Abelian flavor symmetries with conserved currents $j_{(\alpha)}^{\mu}, \alpha=1,2,3$, corresponding to the flavor charges of $\left(\mathcal{Z}_{1}, \mathcal{Z}_{2}, \mathcal{W}_{1}, \mathcal{W}_{2}\right)$ being $^{33}\left(\frac{1}{2}, \frac{1}{2},-\frac{1}{2},-\frac{1}{2}\right),\left(\frac{1}{2},-\frac{1}{2}, \frac{1}{2},-\frac{1}{2}\right)$, and $\left(\frac{1}{2},-\frac{1}{2},-\frac{1}{2}, \frac{1}{2}\right)$. Correspondingly, there is a 3 -parameter family of R-charge assignments

$$
\begin{aligned}
r_{\mathcal{Z}_{1}} & =\frac{1}{2}\left(1+t_{1}+t_{2}+t_{3}\right), \\
r_{\mathcal{Z}_{2}} & =\frac{1}{2}\left(1+t_{1}-t_{2}-t_{3}\right), \\
r_{\mathcal{W}_{1}} & =\frac{1}{2}\left(1-t_{1}+t_{2}-t_{3}\right), \\
r_{\mathcal{W}_{2}} & =\frac{1}{2}\left(1-t_{1}-t_{2}+t_{3}\right),
\end{aligned}
$$

that can be used to couple the theory to $S^{3}$ and compute the 2- and 3-point functions of the canonically normalized operators $J_{(\alpha)}$ in the same multiplet as $j_{(\alpha)}^{\mu}$ using (A.2) and (A.3).

For $N=1$, it is straightforward to apply the formulas in [17] to obtain

$$
F_{\text {free }}=-\ell\left(1-r_{\mathcal{Z}_{1}}\right)-\ell\left(1-r_{\mathcal{Z}_{2}}\right)-\ell\left(1-r_{\mathcal{W}_{1}}\right)-\ell\left(1-r_{\mathcal{W}_{2}}\right)
$$

where $\ell(z)$ is a function defined in [17] obeying $\ell^{\prime}(z)=-\pi z \cot (\pi z)$ and $\ell(0)=0$. An expansion at small $t_{\alpha}$ gives

$$
F_{\text {free }}=2 \log 2-\frac{\pi^{2}}{4}\left(t_{1}^{2}+t_{2}^{2}+t_{3}^{2}\right)+\pi^{2} t_{1} t_{2} t_{3}+O\left(t^{4}\right) .
$$

From (A.2) and (A.3) we obtain

$$
\begin{aligned}
\left\langle J_{(\alpha)}\left(\vec{x}_{1}\right) J_{(\beta)}\left(\vec{x}_{2}\right)\right\rangle_{\text {free }} & =\frac{\delta_{\alpha \beta}}{(4 \pi)^{2}\left|\vec{x}_{1}-\vec{x}_{2}\right|^{2}}, \\
\left\langle J_{(1)}\left(\vec{x}_{1}\right) J_{(2)}\left(\vec{x}_{2}\right) J_{(3)}\left(\vec{x}_{3}\right)\right\rangle_{\text {free }} & =\frac{1}{(4 \pi)^{3}\left|\vec{x}_{1}-\vec{x}_{2}\right|\left|\vec{x}_{1}-\vec{x}_{3}\right|\left|\vec{x}_{2}-\vec{x}_{3}\right|} .
\end{aligned}
$$

This result agrees with (2.25) when using the matrices in (2.17) and $c_{T}=16$.

\footnotetext{
${ }^{33}$ This normalization of the $\mathrm{U}(1)^{3}$ charges was chosen such that it agrees with the normalization in section 2.2 .
} 
At large $N$, it was shown in [16] that

$$
F=\frac{4 \sqrt{2} \pi N^{3 / 2}}{3} \sqrt{r_{\mathcal{Z}_{1}} r_{\mathcal{Z}_{2}} r_{\mathcal{W}_{1}} r_{\mathcal{W}_{2}}}+O\left(N^{1 / 2}\right)
$$

Expanding at small $t_{\alpha}$, we have

$$
F=\frac{4 \sqrt{2} \pi N^{3 / 2}}{3}\left[\frac{1}{4}-\frac{1}{4}\left(t_{1}^{2}+t_{2}^{2}+t_{3}^{2}\right)+t_{1} t_{2} t_{3}+O\left(t^{4}\right)\right]+O\left(N^{1 / 2}\right) .
$$

From (A.2) and (A.3) we extract

$$
\begin{aligned}
\left\langle J_{(\alpha)}\left(\vec{x}_{1}\right) J_{(\beta)}\left(\vec{x}_{2}\right)\right\rangle & =\frac{4 \sqrt{2} N^{3 / 2}}{3 \pi} \frac{\delta_{\alpha \beta}}{(4 \pi)^{2}\left|\vec{x}_{1}-\vec{x}_{2}\right|^{2}}+O\left(N^{1 / 2}\right), \\
\left\langle J_{(1)}\left(\vec{x}_{1}\right) J_{(2)}\left(\vec{x}_{2}\right) J_{(3)}\left(\vec{x}_{3}\right)\right\rangle & =\frac{4 \sqrt{2} N^{3 / 2}}{3 \pi} \frac{1}{(4 \pi)^{3}\left|\vec{x}_{1}-\vec{x}_{2}\right|\left|\vec{x}_{1}-\vec{x}_{3}\right|\left|\vec{x}_{2}-\vec{x}_{3}\right|}+O\left(N^{1 / 2}\right) .
\end{aligned}
$$

Using

$$
\frac{4 \sqrt{2} N^{3 / 2}}{3 \pi} \approx \frac{c_{T}}{16} \approx \frac{2 L^{2}}{\pi G_{4}},
$$

(see, for example, [47]) we see that these expressions agree with (2.29)-(2.30).

\section{B Some details of the derivation of (3.20)}

Below, the Killing spinor is assumed to be Majorana. We start by writing all terms in $\delta S_{\text {kin }}$ involving the $P_{R} \chi$ projection of the spinor field and then add the conjugate terms.

$$
\begin{aligned}
\delta S_{\text {kin } P_{R} \chi}=\int d^{4} x \sqrt{-g}[ & -\partial_{\mu}\left(\bar{\epsilon} P_{R} \chi\right) \partial^{\mu} z-\frac{1}{2} \bar{\chi} \gamma^{\mu} \nabla_{\mu}\left(P_{L}(\not \nabla z+F) \epsilon\right) \\
& +\frac{1}{2} \bar{\epsilon}(\not \nabla z-F) \gamma^{\mu} \nabla_{\mu} P_{R} \chi+\left(\bar{\epsilon} \gamma^{\mu} \nabla_{\mu} P_{R} \chi\right)\left(F+\frac{z}{L}\right) \\
& \left.+2\left(\bar{\epsilon} P_{R} \chi\right) \frac{z}{L^{2}}\right] .
\end{aligned}
$$

The 3 terms involving $F$ are

$$
-\frac{1}{2} \bar{\chi} \gamma^{\mu} \nabla_{\mu}\left(P_{L} F \epsilon\right)-\frac{1}{2} \bar{\epsilon} F \gamma^{\mu} \nabla_{\mu} P_{R} \chi+\left(\bar{\epsilon} \gamma^{\mu} \nabla_{\mu} P_{R} \chi\right) F .
$$

After a Majorana flip of the first term and adding the last two terms we recognize the total derivative

$$
\frac{1}{2} \nabla_{\mu}\left(\bar{\epsilon} F \gamma^{\mu} P_{R} \chi\right)
$$

This becomes the $P_{R} \chi$ part of the last term in (3.20). Next use

$$
\gamma^{\mu} \gamma^{\nu} \nabla_{\mu}\left(\partial_{\nu} z \epsilon\right)=\square z \epsilon+\gamma^{\mu} \gamma^{\nu} \partial_{\nu} z \nabla_{\mu} \epsilon=\square z \epsilon+(1 / L) \not \nabla z \epsilon,
$$

in which the Killing spinor equation and a standard $\gamma$-matrix identity are used to write the last term. This relation is used in the second term of (B.1) and, after partial integration, in the third term also. After partial integration in the first term, one see that the 3 terms 
containing $\square z$ cancel. One is left with the two total derivatives from the partial integrations plus terms in $1 / L$ and $1 / L^{2}$, namely

$$
\begin{aligned}
\delta S_{\text {kin } P_{R} \chi}=\int d^{4} x \sqrt{-g}[ & -\nabla_{\mu}\left(\bar{\epsilon} P_{R} \chi \partial^{\mu} z\right)+\frac{1}{2} \nabla_{\mu}\left(\bar{\epsilon} \not \nabla z \gamma^{\mu} P_{R} \chi\right) \\
& \left.+\frac{1}{L} \bar{\epsilon}\left[\not \nabla z P_{R} \chi+z \gamma^{\mu} \nabla_{\mu} P_{R} \chi\right]+2 \frac{z}{L^{2}} \bar{\epsilon} P_{R} \chi\right] .
\end{aligned}
$$

The terms inside the square bracket add to the derivative of the product $z P_{R} \chi$. This is partially integrated giving another total derivative plus terms that vanish by Killing spinor equation.

\section{Truncating the $\mathcal{N}=8$ theory}

\section{C.1 Truncations and flows}

There are many important instances in which the full $\mathcal{N}=8$ theory is truncated to a subsector with a reduced amount of supersymmetry. To define the reduced, or truncated, theory we introduce a projection matrix, $\Pi^{i}{ }_{j}$, whose task will be to project onto the supersymmetries of interest. Specifically, the supersymmetries in the truncation are given by:

$$
\Pi_{j}^{i} \varepsilon^{j}=\varepsilon^{i}, \quad \Pi^{j}{ }_{i} \varepsilon_{j}=\varepsilon_{i}, \quad \Pi_{j}^{i} \Pi_{k}^{j}=\Pi_{k}^{i}, \quad \text { with } \quad p \equiv \Pi_{i}^{i}=\operatorname{Tr}(\Pi) .
$$

We are thus truncating to a theory with $p$ supersymmetries.

In the second part of this appendix we show, in particular, that if $\Pi^{i}{ }_{j}$ is a projector acting on the supersymmetries in such a way that it reduces their number to $p$ in a manner consistent with (5.21) and (5.22) then the boundary counterterm action is simply:

$$
S_{\mathrm{b}, \text { truncated }}=-\frac{2}{p L} \int d^{3} x e^{3 r_{0} / L} \operatorname{Tr} \sqrt{\Pi A_{1} A_{1}^{\dagger}} .
$$

Indeed, in many instances, ${ }^{34} \sqrt{\Pi A_{1} A_{1}^{\dagger}}$ is simply diagonal on the relevant subspace and has eigenvalues $e^{\mathcal{K} / 2} W$. Thus (C.2) yields the same result as in (3.4).

It is also important to note that (C.2) represents a sum over a subset of $p$ of the eigenvalues of $A_{1} A_{1}^{\dagger}$. From (5.12) one sees that, at quadratic order in $\phi$, the eigenvalues are all the same while at cubic order they will depend on details of the truncation. Thus, as one would expect, to quadratic (divergent) order, the counterterms are universal ${ }^{35}$ but the finite counterterms depend upon the details of the supersymmetry of the truncated theory. In particular, the truncation will generically break $\mathrm{SO}(8)$ to $\mathrm{SO}(p)$, or perhaps even some subgroup of $\mathrm{SO}(p)$. Thus the form of the finite counterterms is no longer bound by $\mathrm{SO}(8)$ invariance, and it is quite possible that the truncated analog of (6.30) might allow some $\alpha(\beta)^{2}$ terms. Indeed, we encountered precisely such terms in sections 3.6-3.8.

We would be remiss if we did not mention flow in the context of the Bogomolny factorization. Flows are solutions that depended solely on $r$ and are thus independent of the

\footnotetext{
${ }^{34}$ For early examples, see [71-75].

${ }^{35}$ The factors of $p$ cancel between the coefficient of (C.2) and the sum in the trace.
} 
boundary directions. Supersymmetric flow solutions preserve some subset of the supersymmetries and the BPS equations can typically be obtained by requiring each squared term in the Bogomolny action to vanish independently. This means that $\mathcal{A}_{a i j k l}=0$ and, from, (C.10):

$$
\mathcal{A}^{\prime} \delta_{i j}= \pm \sqrt{2} g \Pi^{k}{ }_{i} D_{k j}, \quad \Pi^{i}{ }_{m} \mathcal{A}_{r}{ }^{m j k l}=\mp 2 g \Pi_{p}^{i} X^{p m} A_{2 m}{ }^{j k l} .
$$

This means that the eigenvalues of $\Pi D$ must all be the same and reduce to essentially a single superpotential, while the second equation in (C.3) takes the form of steepest descents on that superpotential, exactly as in section 3.1. Also see, for example, [62, 63, 70].

\section{C.2 Calculation of the counterterms}

Consistency with (5.21) requires:

$$
D_{\mu} \Pi^{i}{ }_{j}=0, \quad \Pi_{k}^{i} A_{1}{ }^{k j}=\Pi^{j}{ }_{k} A_{1}{ }^{i k} \Rightarrow \Pi_{k}^{i} \Pi^{j}{ }_{m} A_{1}{ }^{k m}=\Pi_{k}^{i} A_{1}{ }^{k j} .
$$

It follows that our truncation must reduce the $A_{1}$ tensor to a $p \times p$ sub-matrix and the gravitino variations are restricted to the components of (5.21) along $\Pi_{k}^{i} \Pi^{j}{ }_{m} A_{1}{ }^{k m}$.

To perform the Bogomolny trick in the truncated theory we need two identities involving the tensors associated with the scalars. First, consider the partial contraction:

$$
\begin{aligned}
\mathcal{A}_{\mu}{ }^{i k l m} \mathcal{A}^{\mu}{ }_{j k l m} & =\frac{1}{576} \epsilon^{i k l m p q r s} \epsilon_{j k l m t u v w} \mathcal{A}_{\mu p q r s} \mathcal{A}^{\mu t u v w}=\frac{5}{4} \delta_{j}^{[i} \delta_{t}^{p} \delta_{u}^{q} \delta_{v}^{r} \delta_{w}^{s]}, \mathcal{A}_{\mu p q r s} \mathcal{A}^{\mu t u v w} \\
& =\frac{1}{4} \delta_{j}^{i} \mathcal{A}_{\mu p q r s t} \mathcal{A}^{\mu p q r s t}-\mathcal{A}^{\mu}{ }_{j k l m} \mathcal{A}_{\mu}{ }^{i k l m}
\end{aligned}
$$

It follows that the self-duality of the kinetic term implies that one has:

$$
\mathcal{A}_{\mu}{ }^{i k l m} \mathcal{A}^{\mu}{ }_{j k l m}=\frac{1}{8} \delta_{j}^{i} \mathcal{A}_{\mu}{ }^{p q r s t} \mathcal{A}^{\mu}{ }_{p q r s t} .
$$

There is also a very similar identity in [1] for the A-tensors:

$$
-\frac{3}{4} A_{1}{ }^{i k} A_{1 k j}+\frac{1}{24} A_{2}{ }^{i}{ }_{k l m} A_{2 j}{ }^{k l m}=\frac{1}{8} \delta_{j}^{i}\left(-\frac{3}{4}\left|A_{1}{ }^{i j}\right|^{2}+\frac{1}{24}\left|A_{2 i}{ }^{j k l}\right|^{2}\right)=\frac{1}{8} \mathcal{P} \delta_{j}^{i} .
$$

Contracting (C.6) and (C.7) with $\Pi^{i}{ }_{j}$ gives

$$
\begin{aligned}
\mathcal{A}_{\mu}{ }^{i j k l} \mathcal{A}^{\mu}{ }_{i j k l} & =\frac{8}{p} \Pi^{j}{ }_{i} \mathcal{A}_{\mu}{ }^{i k l m} \mathcal{A}^{\mu}{ }_{j k l m} \\
\mathcal{P} & =\frac{8}{p}\left(-\frac{3}{4} \Pi^{j}{ }_{i} A_{1}{ }^{i k} A_{1 k j}+\frac{1}{24} \Pi^{j}{ }_{i} A_{2}{ }^{i}{ }_{k l m} A_{2 j}{ }^{k l m}\right),
\end{aligned}
$$


One can now complete the square, exactly as in section 6 , but now on the truncated subsystem:

$$
\begin{aligned}
S_{B}= & \int d^{4} x e^{3 A}\left[3\left(\mathcal{A}^{\prime}\right)^{2}+\frac{3}{4} g^{2}\left|A_{1}{ }^{i j}\right|^{2}-\frac{1}{96} \mathcal{A}_{r}{ }^{i j k l} \mathcal{A}_{r i j k l}-\frac{1}{24} g^{2}\left|A_{2 i}{ }^{j k l}\right|^{2}\right] \\
= & \int d^{4} x e^{3 A}\left[3\left(\mathcal{A}^{\prime}\right)^{2}+\frac{8}{p}\left(\frac{3}{4} g^{2} \Pi^{j}{ }_{i} A_{1}{ }^{i k} A_{1 k j}-\frac{1}{96} \Pi^{j}{ }_{i} \mathcal{A}_{r}{ }^{i k l m} \mathcal{A}_{r j k l m}\right.\right. \\
& \left.\left.\quad+\frac{1}{24} g^{2} \Pi^{j}{ }_{i} A_{2}{ }^{i} k l m A_{2 j}{ }^{k l m}\right)\right] \\
= & \frac{1}{p} \int d^{4} x e^{3 A}\left[3\left|\mathcal{A}^{\prime} X_{i j} \mp \sqrt{2} g \Pi^{k}{ }_{i} A_{1 k j}\right|^{2}-\frac{1}{12}\left|\Pi^{i}{ }_{m} \mathcal{A}_{r}{ }^{m j k l} \pm 2 g X^{i m} A_{2 m}{ }^{j k l}\right|^{2}\right. \\
& \left. \pm \sqrt{2} g\left(X^{i j} D_{r}\left(e^{3 A} A_{1 i j}\right)+X_{i j} D_{r}\left(e^{3 A} A_{1}{ }^{i j}\right)\right)\right] .
\end{aligned}
$$

Here the matrices, $X_{i j}=\left(X^{i j}\right)^{*}$, are again allowed to be dynamical but satisfy:

$$
X_{i j}=X_{j i}, \quad X_{i j} X^{k j}=\Pi^{k}{ }_{i}, \quad \Pi_{k}^{i} X^{k j}=X^{i j}, \quad \Pi^{j}{ }_{k} X^{i k}=X^{i j} .
$$

That is, it is an $\mathrm{SU}(p)$ matrix on the remaining supersymmetries. As in section 6, we choose $X$ so as to diagonalize $A_{1}$ on the subspace defined by $\Pi$, and the same arguments lead to a counterterm action:

$$
S_{\text {s-ct,truncated }}=-\frac{2}{p L} \int d^{3} x e^{3 r_{0} / L} \operatorname{Tr} \sqrt{\Pi A_{1} A_{1}^{\dagger}} .
$$

In particular, for truncations to $\mathcal{N}=1$ or $\mathcal{N}=2$ supersymmetric theories, the superpotential emerges as one or two, respectively, of the eigenvalues of $A_{1} A_{1}^{\dagger}$ while the other eigenvalues of this matrix play no role in the supersymmetry of the theory. (These other eigenvalues give mass to the gravitini for the broken supersymmetries.) Thus the projection by $\Pi$ in (C.12) onto the subspace of residual supersymmetries is an essential part of getting the correct supersymmetric boundary terms. Indeed, for such truncations, this projection extracts the superpotential terms and thus generates boundary terms exactly of the form (3.6).

\section{Some identities for $\mathrm{SO}(8)$ (anti-)self-dual tensors}

Let $\alpha^{i j k l}=\alpha^{[i j k l]_{+}}$be a self-dual and $\beta^{i j k l}=\beta^{[i j k l]_{-}}$an anti-self-dual real $\mathrm{SO}(8)$ tensor,

$$
\alpha^{i j k l}=\frac{1}{24} \eta^{i j k l m n p q} \alpha^{m n p q}, \quad \beta^{i j k l}=-\frac{1}{24} \eta^{i j k l m n p q} \beta^{m n p q} .
$$


By a repeated use of (D.1) together with the contraction identities for the completely antisymmetric symbol, $\eta^{i j k l m n p q}$, one can prove the following identities (see, e.g., [78]):

$$
\begin{array}{rlr}
\alpha^{i j k l} \beta^{i j k l} & =0 \\
\alpha^{i k l m} \alpha^{j k l m} & =\frac{1}{8} \delta^{i j} \alpha^{k l m n} \alpha^{k l m n}, \\
\beta^{i k l m} \beta^{j k l m} & =\frac{1}{8} \delta^{i j} \beta^{k l m n} \beta^{k l m n}, \\
\alpha^{i k l m} \beta^{j k l m} & =\beta^{i k l m} \alpha^{j k l m}, \\
\alpha^{m n[i j} \alpha^{k l m n} & =\alpha^{m n[i j} \alpha^{k l] m n} \quad & \\
\beta^{m n[i j} \beta^{k] l m n} & =\beta^{m n[i j} \beta^{k l] m n} \quad & \text { self-dual }, \\
\alpha^{m n[i j} \beta^{k] l m n} & =-\beta^{m n[i j} \alpha^{k] l m n}, &
\end{array}
$$

and

$$
\begin{aligned}
\eta_{k l m n p q r s} \alpha_{(1)}{ }^{i k l m} \alpha_{(1)}{ }^{j n p q}= & 18 \alpha_{(1)}{ }^{i j m n} \alpha_{(1)}{ }^{r s m n} \\
& +6 \delta_{s i} \alpha_{(1)}{ }^{j m n p} \alpha_{(1)}{ }^{r m n p}-6 \delta_{r i} \alpha_{(1)}{ }^{j m n p} \alpha_{(1)}{ }^{s m n p} \\
= & 18 \alpha_{(1)}{ }^{i j m n} \alpha_{(1)}{ }^{r s m n}+\frac{3}{4}\left(\delta_{i r} \delta_{j s}-\delta_{i r} \delta_{j s}\right) \alpha_{(1)}{ }^{m n p q} \alpha_{(1)}{ }^{m n p q}, \\
\eta_{k l m n p q r s} \alpha_{(1)}{ }^{i k l m} \beta_{(1)}{ }^{j n p q}= & -18 \alpha_{(1)}{ }^{i j m n} \beta_{(1)}{ }^{r s m n} \\
& +6 \delta_{s j} \alpha_{(1)}^{i m n p} \beta_{(1)}^{r m n p}-6 \delta_{r j} \alpha_{(1)}{ }^{i m n p} \beta_{(1)}{ }^{s m n p} \\
= & 18 \alpha_{(1)}{ }^{r m n} \beta_{(1)}{ }^{i j m n} \\
& +6 \delta_{s i} \alpha_{(1)}^{r m n p} \beta_{(1)}{ }^{j m n p}-6 \delta_{r i} \alpha_{(1)}{ }^{s m n p} \beta_{(1)}{ }^{j m n p}
\end{aligned}
$$

which are used in sections 6 and 7 .

\section{E The $\mathrm{U}(1)^{3}$-invariant truncation in [14]}

The scalar sector of the $\mathrm{U}(1)^{3}$-invariant truncation of $\mathcal{N}=8$ supergravity $^{36}$ studied in [14], in the notation of the present paper, is given by

$$
\begin{aligned}
& \alpha^{1234}=\alpha^{5678}=\rho_{1} \cos \theta_{1}, \\
& \alpha^{1256}=\alpha^{3478}=\rho_{2} \cos \theta_{2}, \\
& \alpha^{3456}=\alpha^{1278}=\rho_{3} \cos \theta_{3},
\end{aligned}
$$

where

$$
z_{\alpha}=\tanh \rho_{\alpha} e^{i \theta_{\alpha}}, \quad \alpha=1,2,3
$$

\footnotetext{
${ }^{36}$ For an early work on this truncation, see [15].
} 
After the change from the $\mathrm{SU}(8)$ to the $\mathrm{SL}(8, \mathbb{R})$ basis, only the diagonal fields, $A^{I I}$, are nonzero and are given by ${ }^{37}$

$$
\begin{aligned}
& A^{11}=A^{77}=\frac{1}{2}\left(\rho_{1} \cos \theta_{1}-\rho_{2} \cos \theta_{2}-\rho_{3} \cos \theta_{3}\right), \\
& A^{22}=A^{88}=\frac{1}{2}\left(\rho_{1} \cos \theta_{1}+\rho_{2} \cos \theta_{2}+\rho_{3} \cos \theta_{3}\right), \\
& A^{33}=A^{66}=\frac{1}{2}\left(-\rho_{1} \cos \theta_{1}-\rho_{2} \cos \theta_{2}+\rho_{3} \cos \theta_{3}\right), \\
& A^{44}=A^{55}=\frac{1}{2}\left(-\rho_{1} \cos \theta_{1}+\rho_{2} \cos \theta_{2}-\rho_{3} \cos \theta_{3}\right) .
\end{aligned}
$$

The qudartic and cubic counterterms are then

$$
\begin{aligned}
-\frac{1}{4 L} A^{I J} A^{I J} & =-\frac{1}{2 L}\left(\rho_{1}^{2} \cos ^{2} \theta_{1}+\rho_{2}^{2} \cos ^{2} \theta_{2}+\rho_{3}^{2} \cos ^{2} \theta_{3}\right) \\
& =-\frac{1}{2 L}\left(z_{1} \bar{z}_{1}+z_{2} \bar{z}_{2}+z_{3} \bar{z}_{3}\right)+\ldots, \\
\frac{1}{6 \sqrt{2} L} A^{I J} A^{J K} A^{K I} & =\frac{1}{\sqrt{2} L} \rho_{1} \rho_{2} \rho_{3} \cos \theta_{1} \cos \theta_{2} \cos \theta_{3} \\
& =\frac{1}{2 \sqrt{2} L}\left(z_{1} z_{2} z_{3}+\bar{z}_{1} \bar{z}_{2} \bar{z}_{3}\right)+\ldots
\end{aligned}
$$

where one must set the pseudoscalars to zero. The ... stand for terms quartic in the fields due to the expansion $\tanh \rho_{\alpha}=\rho_{\alpha}+\ldots$

Open Access. This article is distributed under the terms of the Creative Commons Attribution License (CC-BY 4.0), which permits any use, distribution and reproduction in any medium, provided the original author(s) and source are credited.

\section{References}

[1] B. de Wit and H. Nicolai, $N=8$ Supergravity, Nucl. Phys. B 208 (1982) 323 [inSPIRE].

[2] V. Pestun, Localization of gauge theory on a four-sphere and supersymmetric Wilson loops, Commun. Math. Phys. 313 (2012) 71 [arXiv:0712.2824] [INSPIRE].

[3] O. Aharony, O. Bergman, D.L. Jafferis and J. Maldacena, $N=6$ superconformal Chern-Simons-matter theories, M2-branes and their gravity duals, JHEP 10 (2008) 091 [arXiv:0806.1218] [INSPIRE].

[4] O. Aharony, O. Bergman and D.L. Jafferis, Fractional M2-branes, JHEP 11 (2008) 043 [arXiv: 0807.4924] [INSPIRE].

[5] J. Bagger and N. Lambert, Modeling Multiple M2's, Phys. Rev. D 75 (2007) 045020 [hep-th/0611108] [INSPIRE].

[6] A. Gustavsson, Algebraic structures on parallel M2-branes, Nucl. Phys. B 811 (2009) 66 [arXiv: 0709.1260] [INSPIRE].

[7] J. Bagger and N. Lambert, Gauge symmetry and supersymmetry of multiple M2-branes, Phys. Rev. D 77 (2008) 065008 [arXiv:0711.0955] [InSPIRE].

\footnotetext{
${ }^{37}$ The particular arrangement of the signs in (E.3) depends on a representation of $\Gamma$-matrices of $\mathrm{SO}(8)$.
} 
[8] J. Bagger and N. Lambert, Comments on multiple M2-branes, JHEP 02 (2008) 105 [arXiv: 0712.3738] [INSPIRE].

[9] D. Bashkirov and A. Kapustin, Supersymmetry enhancement by monopole operators, JHEP 05 (2011) 015 [arXiv: 1007.4861] [INSPIRE].

[10] M.K. Benna, I.R. Klebanov and T. Klose, Charges of Monopole Operators in Chern-Simons Yang-Mills Theory, JHEP 01 (2010) 110 [arXiv:0906.3008] [INSPIRE].

[11] A. Gustavsson and S.-J. Rey, Enhanced $N=8$ Supersymmetry of ABJM Theory on $\mathbb{R}^{8}$ and $\mathbb{R}^{8} / \mathbb{Z}_{2}$, arXiv:0906.3568 [INSPIRE].

[12] M. Benna, I. Klebanov, T. Klose and M. Smedback, Superconformal Chern-Simons Theories and $A d S_{4} / C F T_{3}$ Correspondence, JHEP 09 (2008) 072 [arXiv:0806.1519] [INSPIRE].

[13] O.-K. Kwon, P. Oh and J. Sohn, Notes on Supersymmetry Enhancement of ABJM Theory, JHEP 08 (2009) 093 [arXiv: 0906.4333] [INSPIRE].

[14] D.Z. Freedman and S.S. Pufu, The holography of F-maximization, JHEP 03 (2014) 135 [arXiv: 1302.7310] [INSPIRE].

[15] M. Cvetič et al., Embedding AdS black holes in ten-dimensions and eleven-dimensions, Nucl. Phys. B 558 (1999) 96 [hep-th/9903214] [INSPIRE].

[16] D.L. Jafferis, I.R. Klebanov, S.S. Pufu and B.R. Safdi, Towards the F-Theorem: $\mathcal{N}=2$ Field Theories on the Three-Sphere, JHEP 06 (2011) 102 [arXiv:1103.1181] [INSPIRE].

[17] D.L. Jafferis, The Exact Superconformal R-Symmetry Extremizes Z, JHEP 05 (2012) 159 [arXiv: 1012.3210] [INSPIRE].

[18] A. Kapustin, B. Willett and I. Yaakov, Exact Results for Wilson Loops in Superconformal Chern-Simons Theories with Matter, JHEP 03 (2010) 089 [arXiv:0909.4559] [INSPIRE].

[19] N. Hama, K. Hosomichi and S. Lee, Notes on SUSY Gauge Theories on Three-Sphere, JHEP 03 (2011) 127 [arXiv: 1012.3512] [INSPIRE].

[20] B. Willett, Localization on three-dimensional manifolds, arXiv:1608.02958 [INSPIRE].

[21] S.S. Pufu, The F-Theorem and F-Maximization, arXiv:1608.02960 [INSPIRE].

[22] P. Breitenlohner and D.Z. Freedman, Stability in Gauged Extended Supergravity, Annals Phys. 144 (1982) 249 [INSPIRE].

[23] M. Bianchi, D.Z. Freedman and K. Skenderis, How to go with an RG flow, JHEP 08 (2001) 041 [hep-th/0105276] [inSPIRE].

[24] M. Bianchi, D.Z. Freedman and K. Skenderis, Holographic renormalization, Nucl. Phys. B 631 (2002) 159 [hep-th/0112119] [INSPIRE].

[25] I.R. Klebanov and E. Witten, AdS/CFT correspondence and symmetry breaking, Nucl. Phys. B 556 (1999) 89 [hep-th/9905104] [INSPIRE].

[26] K. Skenderis and P.K. Townsend, Gravitational stability and renormalization group flow, Phys. Lett. B 468 (1999) 46 [hep-th/9909070] [INSPIRE].

[27] M. Henningson and K. Sfetsos, Spinors and the AdS/CFT correspondence, Phys. Lett. B 431 (1998) 63 [hep-th/9803251] [INSPIRE].

[28] W. Mueck and K.S. Viswanathan, Conformal field theory correlators from classical field theory on anti-de Sitter space. 2. Vector and spinor fields, Phys. Rev. D 58 (1998) 106006 [hep-th/9805145] [INSPIRE]. 
[29] G.E. Arutyunov and S.A. Frolov, On the origin of supergravity boundary terms in the AdS/CFT correspondence, Nucl. Phys. B 544 (1999) 576 [hep-th/9806216] [InSPIRE].

[30] M. Henneaux, Boundary terms in the AdS/CFT correspondence for spinor fields, hep-th/9902137 [INSPIRE].

[31] E. D'Hoker, D.Z. Freedman, S.D. Mathur, A. Matusis and L. Rastelli, Extremal correlators in the AdS/CFT correspondence, hep-th/9908160 [INSPIRE].

[32] F. Bastianelli and R. Zucchini, Three point functions of chiral primary operators in $D=3$, $N=8$ and $D=6, N=(2,0) S C F T$ at large- $N$, Phys. Lett. B 467 (1999) 61 [hep-th/9907047] [INSPIRE].

[33] M. Bianchi and A. Marchetti, Holographic three point functions: One step beyond the tradition, Nucl. Phys. B 686 (2004) 261 [hep-th/0302019] [INSPIRE].

[34] M. Bianchi, M. Prisco and W. Mueck, New results on holographic three point functions, JHEP 11 (2003) 052 [hep-th/0310129] [INSPIRE].

[35] A. Bissi, C. Kristjansen, A. Martirosyan and M. Orselli, On Three-point Functions in the $A d S_{4} / C F T_{3}$ Correspondence, JHEP 01 (2013) 137 [arXiv:1211.1359] [InSPIRE].

[36] G. Festuccia and N. Seiberg, Rigid Supersymmetric Theories in Curved Superspace, JHEP 06 (2011) 114 [arXiv: 1105.0689] [INSPIRE].

[37] E. Witten and D.I. Olive, Supersymmetry Algebras That Include Topological Charges, Phys. Lett. B 78 (1978) 97 [INSPIRE].

[38] P. Fendley, S.D. Mathur, C. Vafa and N.P. Warner, Integrable Deformations and Scattering Matrices for the $N=2$ Supersymmetric Discrete Series, Phys. Lett. B 243 (1990) 257 [INSPIRE].

[39] N.P. Warner, Supersymmetry in boundary integrable models, Nucl. Phys. B 450 (1995) 663 [hep-th/9506064] [INSPIRE].

[40] D.Z. Freedman, S.D. Mathur, A. Matusis and L. Rastelli, Correlation functions in the $C F T_{d} / A d S_{d+1}$ correspondence, Nucl. Phys. B 546 (1999) 96 [hep-th/9804058] [INSPIRE].

[41] I.R. Klebanov and A.M. Polyakov, AdS dual of the critical $O(N)$ vector model, Phys. Lett. B 550 (2002) 213 [hep-th/0210114] [INSPIRE].

[42] S. Giombi and X. Yin, Higher Spin Gauge Theory and Holography: The Three-Point Functions, JHEP 09 (2010) 115 [arXiv:0912.3462] [INSPIRE].

[43] S. Lee, S. Minwalla, M. Rangamani and N. Seiberg, Three point functions of chiral operators in $D=4, N=4 S Y M$ at large- $N$, Adv. Theor. Math. Phys. 2 (1998) 697 [hep-th/9806074] [INSPIRE].

[44] E. D'Hoker, D.Z. Freedman and W. Skiba, Field theory tests for correlators in the AdS/CFT correspondence, Phys. Rev. D 59 (1999) 045008 [hep-th/9807098] [INSPIRE].

[45] C. Closset, T.T. Dumitrescu, G. Festuccia, Z. Komargodski and N. Seiberg, Contact Terms, Unitarity and F-Maximization in Three-Dimensional Superconformal Theories, JHEP 10 (2012) 053 [arXiv: 1205.4142] [INSPIRE].

[46] T.T. Dumitrescu, An introduction to supersymmetric field theories in curved space, arXiv: 1608.02957 [INSPIRE].

[47] S.M. Chester, J. Lee, S.S. Pufu and R. Yacoby, The $\mathcal{N}=8$ superconformal bootstrap in three dimensions, JHEP 09 (2014) 143 [arXiv:1406.4814] [INSPIRE]. 
[48] M. Dedushenko, S.S. Pufu and R. Yacoby, A one-dimensional theory for Higgs branch operators, arXiv: 1610.00740 [INSPIRE].

[49] D.V. Belyaev and P. van Nieuwenhuizen, Simple D $=4$ supergravity with a boundary, JHEP 09 (2008) 069 [arXiv: 0806.4723] [INSPIRE].

[50] P. van Nieuwenhuizen, A. Rebhan, D.V. Vassilevich and R. Wimmer, Boundary terms in supergravity and supersymmetry, Int. J. Mod. Phys. D 15 (2006) 1643 [hep-th/0606075] [INSPIRE].

[51] P. van Nieuwenhuizen and D.V. Vassilevich, Consistent boundary conditions for supergravity, Class. Quant. Grav. 22 (2005) 5029 [hep-th/0507172] [INSPIRE].

[52] S.W. Hawking, The Boundary Conditions for Gauged Supergravity, Phys. Lett. B 126 (1983) 175 [INSPIRE].

[53] D. Grumiller and P. van Nieuwenhuizen, Holographic counterterms from local supersymmetry without boundary conditions, Phys. Lett. B 682 (2010) 462 [arXiv:0908.3486] [INSPIRE].

[54] D.V. Belyaev and P. van Nieuwenhuizen, Rigid supersymmetry with boundaries, JHEP 04 (2008) 008 [arXiv: 0801.2377] [INSPIRE].

[55] S. Corley, The massless gravitino and the AdS/CFT correspondence, Phys. Rev. D 59 (1999) 086003 [hep-th/9808184] [INSPIRE].

[56] A. Volovich, Rarita-Schwinger field in the AdS/CFT correspondence, JHEP 09 (1998) 022 [hep-th/9809009] [INSPIRE].

[57] R.C. Rashkov, Note on the boundary terms in AdS/CFT correspondence for Rarita-Schwinger field, Mod. Phys. Lett. A 14 (1999) 1783 [hep-th/9904098] [InSPIRE].

[58] A.J. Amsel and G. Compere, Supergravity at the boundary of AdS supergravity, Phys. Rev. D 79 (2009) 085006 [arXiv:0901.3609] [INSPIRE].

[59] L. Andrianopoli and R. D'Auria, $N=1$ and $N=2$ pure supergravities on a manifold with boundary, JHEP 08 (2014) 012 [arXiv:1405.2010] [INSPIRE].

[60] D. Z. Freedman and A. Van Proeyen, Supergravity, Cambridge University Press, Cambridge (2012).

[61] C.J.C. Burges, D.Z. Freedman, S. Davis and G.W. Gibbons, Supersymmetry in Anti-de Sitter Space, Annals Phys. 167 (1986) 285 [InSPIRE].

[62] N. Bobev, K. Pilch and N.P. Warner, Supersymmetric Janus Solutions in Four Dimensions, JHEP 06 (2014) 058 [arXiv: 1311.4883] [InSPIRE].

[63] K. Pilch, A. Tyukov and N.P. Warner, $\mathcal{N}=2$ Supersymmetric Janus Solutions and Flows: From Gauged Supergravity to M-theory, JHEP 05 (2016) 005 [arXiv: 1510.08090] [INSPIRE].

[64] K. Pilch, A. Tyukov and N.P. Warner, Flowing to Higher Dimensions: A New Strongly-Coupled Phase on M2 Branes, JHEP 11 (2015) 170 [arXiv:1506.01045] [INSPIRE].

[65] B. de Wit and $\mathrm{H}$. Nicolai, $N=8$ Supergravity with Local $\mathrm{SO}(8) \times \mathrm{SU}(8)$ Invariance, Phys. Lett. B 108 (1982) 285 [inSPIRE].

[66] E. Cremmer and B. Julia, The SO(8) Supergravity, Nucl. Phys. B 159 (1979) 141 [inSPIRE].

[67] M.J. Duff, B.E.W. Nilsson and C.N. Pope, Kaluza-Klein Supergravity, Phys. Rept. 130 (1986) 1 [INSPIRE].

[68] E.B. Bogomolny, Stability of Classical Solutions, Sov. J. Nucl. Phys. 24 (1976) 449 [InSPIRE]. 
[69] M.K. Prasad and C.M. Sommerfield, An Exact Classical Solution for the 't Hooft Monopole and the Julia-Zee Dyon, Phys. Rev. Lett. 35 (1975) 760 [InSPIRE].

[70] C.N. Pope and N.P. Warner, A dielectric flow solution with maximal supersymmetry, JHEP 04 (2004) 011 [hep-th/0304132] [INSPIRE].

[71] C.-h. Ahn and K. Woo, Supersymmetric domain wall and $R G$ flow from 4-dimensional gauged $N=8$ supergravity, Nucl. Phys. B 599 (2001) 83 [hep-th/0011121] [InSPIRE].

[72] R. Corrado, K. Pilch and N.P. Warner, An $N=2$ supersymmetric membrane flow, Nucl. Phys. B 629 (2002) 74 [hep-th/0107220] [INSPIRE].

[73] C.-h. Ahn and K.-s. Woo, Domain wall and membrane flow from other gauged $D=4, N=8$ supergravity. Part 1, Nucl. Phys. B 634 (2002) 141 [hep-th/0109010] [InSPIRE].

[74] C.-h. Ahn and T. Itoh, An $N=1$ supersymmetric G-2 invariant flow in M-theory, Nucl. Phys. B 627 (2002) 45 [hep-th/0112010] [INSPIRE].

[75] C.-h. Ahn and K.-s. Woo, Domain wall from gauged $D=4, N=8$ supergravity. Part 2, JHEP 11 (2003) 014 [hep-th/0209128] [INSPIRE].

[76] R.A. Horn and C.R. Johnson, Matrix Analysis, Cambridge University Press, Cambridge (1985).

[77] R. Slansky, Group Theory for Unified Model Building, Phys. Rept. 79 (1981) 1 [InSPIRE].

[78] B. de Wit, Properties of SO(8) Extended Supergravity, Nucl. Phys. B 158 (1979) 189 [INSPIRE]. 